\author{
Universidade de São Paulo \\ Instituto de Astronomia, Geofísica e Ciências Atmosféricas
}

Departamento de Astronomia

George José Martins Zilioti

\title{
Um Estudo Sobre a Tensão Supernova - Radiação Cósmica de Fundo e o Decaimento do Vácuo
}

São Paulo 

George José Martins Zilioti

\section{Um Estudo Sobre a Tensão Supernova - Radiação Cósmica de Fundo e o Decaimento do Vácuo}

Dissertação apresentada ao Departamento de Astronomia do Instituto de Astronomia, Geofísica

e Ciências Atmosféricas da Universidade de São Paulo como requisito parcial para a obtenção do título de Mestre em Ciências.

Área de Concentração: Astronomia

Orientador: Prof. Dr. José Ademir Sales de Lima

São Paulo 



\section{Agradecimentos}

Agradeço primeiramente à minha família, que sempre esteve do meu lado, à minha namorada, Cíntia, pela companhia e apoio,

Ao meu orientador, José Ademir Sales de Lima, pelo tema proposto e por sempre estar disponível para esclarecer minhas dúvidas,

Aos meus colegas do IAG e do grupo de cosmologia,

Aos professores e funcionários do IAG e da USP, por fornecer um serviço essencial não só a universidade, mas a todo o país,

E por fim à Capes, pelo apoio financeiro.

Esta dissertação foi escrita em IATEX com a classe IAGTESE, para teses e dissertações do IAG. 



\section{Resumo}

Neste trabalho analisamos algumas consequências físicas de uma cosmologia acelerada com interação no chamado setor cósmico escuro (energia escura + matéria escura fria). A componente de energia escura é representada por uma densidade de energia do vácuo que varia com o tempo e cuja lei de decaimento tem a seguinte forma: $\Lambda=\Lambda_{0}+3 \alpha / a^{2}$, onde $\Lambda_{0}$ é o termo de vácuo usual, $\alpha$ é um parâmetro livre e $a(t)$ o fator de escala. Nesse contexto discutimos a tensão existente entre os dados de Supernovas (que preferem um Universo fechado, $\Omega_{\kappa}>0$ ) e os dados da radiação cósmica de fundo que favorecem um Universo espacialmente plano $\left(\Omega_{\kappa}=0\right)$. Considerando que o termo variável simula uma curvatura (pois ambos possuem a mesma dependência no fator de escala), mostramos que sua contribuição atua no sentido de aliviar a tensão SNe Ia-CMB existente no modelo de concordância cósmica padrão $(\Lambda C D M, \alpha=0)$. O modelo resolve o problema da idade do Universo e para $a>>1$, tal como ocorre com $\Lambda C D M$, também evolui para um estágio de Sitter. O parâmetro $\alpha$ é limitado através de uma análise estatística conjunta envolvendo dados de Supernovas, CMB (shift parameter) e oscilações acústicas dos bárions (BAO). Separando o termo de vácuo em duas componentes $\left(\Omega_{\Lambda 0}\right.$ e $\left.\Omega_{\alpha}\right)$ um teste $\chi^{2}$ fornece os seguintes valores para o modelo plano: $\Omega_{m 0}=0,27 \pm 0,02, \Omega_{\Lambda 0}=0,74 \pm 0,02 \mathrm{e}$ $\Omega_{\alpha 0}=-0,01 \pm 0,03$. 



\section{Abstract}

In this work we analyze some physical consequences of an accelerating cosmology endowed with interaction in the cosmic dark sector (dark energy + cold dark matter). The dark energy component is represented by a time-dependent vacuum energy whose decay law has the following form: $\Lambda=\Lambda_{0}+3 \alpha / a^{2}$, where $\Lambda_{0}$ is the standard vacuum term, $\alpha$ is a free parameter and $a(t)$ is the scale factor. In this context we discuss the existing tension between Supernovas (SNe Ia, which prefer a closed Universe, $\Omega_{\kappa}>0$ ) and the cosmic background radiation (CMB) data (which are favoring a spatially flat Universe, $\Omega_{\kappa}=0$ ). By considering that the variable $\Lambda$-term mimics a curvature (since both terms have the same dependence on the scale factor), we show that its contribution helps to alleviate the tension SNe Ia-CMB existing in the standard cosmic concordance model. The present model solves the age of the Universe problem and for $a>>1$, it also evolves to a de Sitter model as occur with the $\Lambda C D M$ scenario. The contribution of the $\alpha$ parameter is limited through a joint statistical analysis involving Supernovas, CMB (shift parameter) and baryon acoustic oscillations (BAO). By separating the variable vacuum term in two components $\left(\Omega_{\Lambda_{0}}\right.$ e $\left.\Omega_{\alpha}\right)$, a $\chi^{2}$ test furnishes the following values for the free parameters of the flat model: $\Omega_{m 0}=0,27 \pm 0,02, \Omega_{\Lambda 0}=0,74 \pm 0,02$ and $\Omega_{\alpha 0}=-0,01 \pm 0,03$. 



\section{Lista de Figuras}

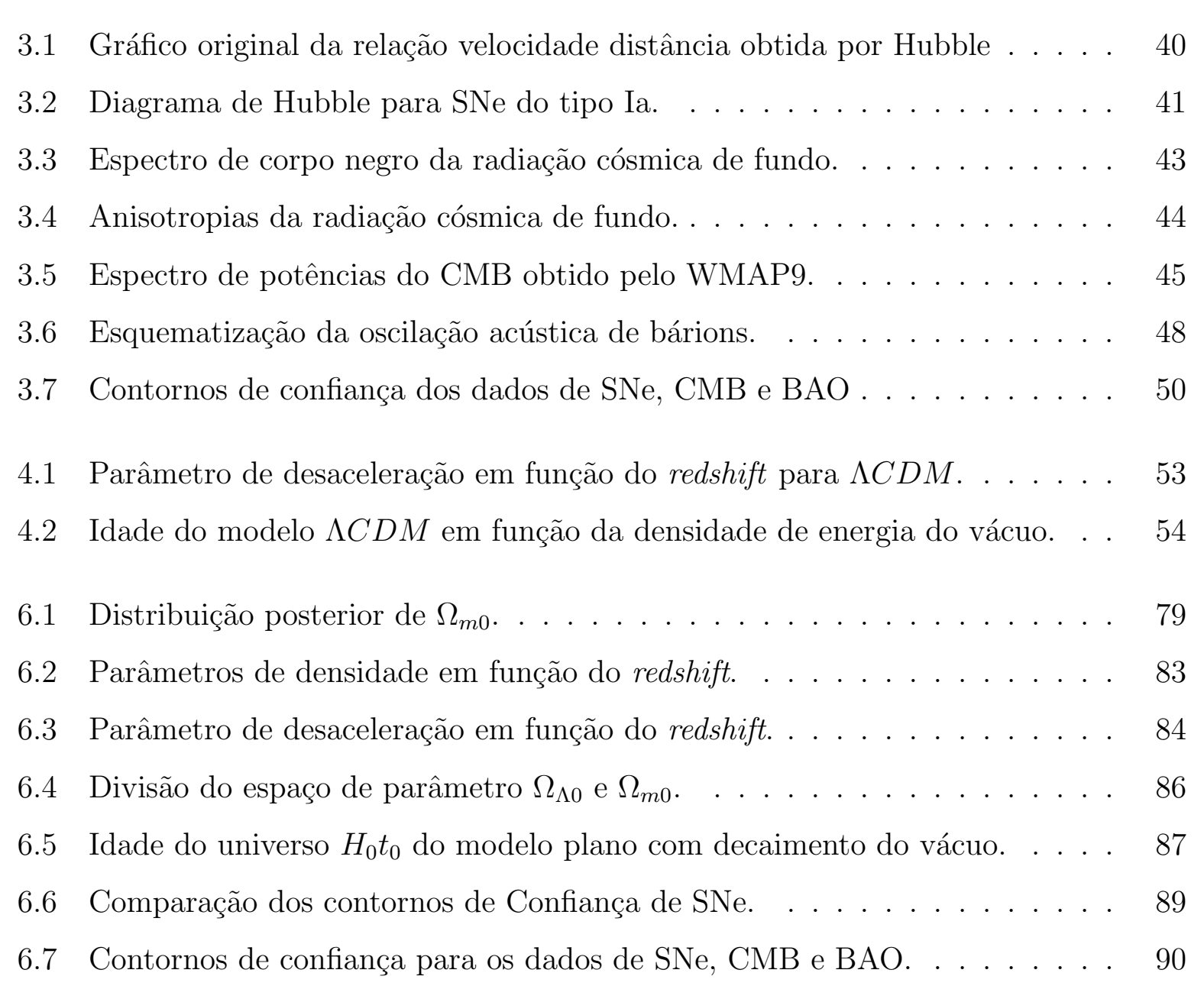





\section{Lista de Tabelas}

3.1 Dados de BAO . . . . . . . . . . . . . . . . . . . . . . . 49

5.1 Leis de Decaimento . . . . . . . . . . . . . . . . . . . . 67

6.1 Valores de $\Delta \chi^{2} \ldots \ldots \ldots \ldots$. . . . . . . . . . . . . . . . . 88

7.1 Comparação de diferentes resultados . . . . . . . . . . . . . . . . . . . . 92 



\section{Sumário}

1. Introducãd . . . . . . . . . . . . . . . . . . . . . . 17

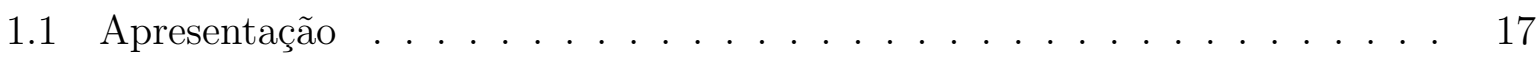

1.2 Organização do Trabalho . . . . . . . . . . . . . . . . . . . . . . . . 18

1.3 Notações . . . . . . . . . . . . . . . . . . . . . . . . . . . . . . . . 19

2. Cosmologias Do Tipo FRW . . . . . . . . . . . . . . . . . . . . . 21

2.1 Equacões de Einstein . . . . . . . . . . . . . . . . . . . . . . . . . 21

2.2 Métrica de FRW e Equacões de Friedmann . . . . . . . . . . . . . . . . . . 22

2.3 Componentes do Universo . . . . . . . . . . . . . . . . . . . . . . . . . . 27

2.3.1 Matéria Bariônica . . . . . . . . . . . . . . . . . . 27

2.3.2 Radiacão. . . . . . . . . . . . . . . . . . . 27

2.3 .3 Matéria Escura . . . . . . . . . . . . . . . . . . . . . 28

2.3 .4 Vácuo . . . . . . . . . . . . . . . . . . . . . 28

2.4 Modelos Cosmológicos . . . . . . . . . . . . . . . . . . . . . . . 31

2.4 .1 Modelo de Einstein . . . . . . . . . . . . . . . . . 31

2.4.2 Modelo de De Sitter . . . . . . . . . . . . . . . . . . . . . 32

2.4 .3 Modelo de Einstein-De Sitter . . . . . . . . . . . . . . 33

3. Distâncias e Cosmologia Observacional . . . . . . . . . . . . . . . . . 35

3.1 Distâncias . . . . . . . . . . . . . . . . . . . . . . . 35

3.1 .1 Distância de Luminosidade . . . . . . . . . . . . . . . . . . . 35

3.1.2 Distância de Diâmetro Angular . . . . . . . . . . . . . . . . 37

3.1 .3 Relacão de Etherington . . . . . . . . . . . . . . . . . 38

3.1.4 Distâncias em Função dos Parâmetros Cosmológicos . . . . . . . . . 38 
3.2 Cosmologia Observacional . . . . . . . . . . . . . . . . . . . . . . . . 39

3.2.1 A Velocidade de Recessão das Galáxias . . . . . . . . . . . . . . . . 39

3.2 .2 Supernovas do Tipo Ia . . . . . . . . . . . . . . . . . . . . 40

$3.2 .3 \quad$ A Radiacão Cósmica de Fundo . . . . . . . . . . . . . . . . . . . . 42

3.2.4 Oscilacões Acústicas de Bárions . . . . . . . . . . . . . . . . . . . . 47

3.2.5 Contornos de Confiança . . . . . . . . . . . . . . . . . . . 49

4. O Modelo de Concordância Cósmica e Energia Escura . . . . . . . . . . . . . . . 51

4.1 o Modelo $\Lambda C D M \ldots \ldots \ldots \ldots \ldots \ldots \ldots$

4.2 Problemas da Constante Cosmológica . . . . . . . . . . . . . . . . . . . . 54

4.2 .1 O Problema Original . . . . . . . . . . . . . . . . . . . . 54

4.2 .2 Novo Problema? . . . . . . . . . . . . . . . 55

4.3 Energia Escura $\ldots \ldots \ldots \ldots$. . . . . . . . . . . . . . . . 56

4.3.1 Modificacões da Relatividade . . . . . . . . . . . . . . . 56

4.3.2 Modelos XCDM . . . . . . . . . . . . . . . 57

4.3.3 Gás de Chaplvgin . . . . . . . . . . . . . . . . . . . . . . 58

4.3 .4 Criacão de Matéria Escura . . . . . . . . . . . . . . . . . 58

4.3 .5 Quintessência . . . . . . . . . . . . . . . . . . . 59

5. Modelos Com Interação no Setor Escurd . . . . . . . . . . . . . . . . . . . . . . 61

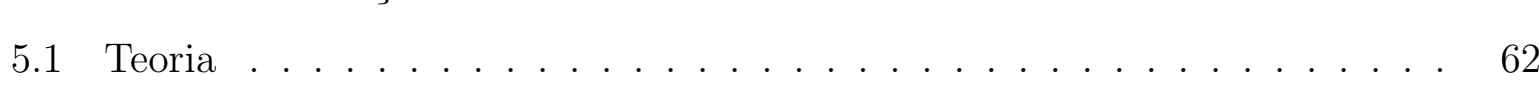

5.2 Problema da Coincidência . . . . . . . . . . . . . . . . . . 65

5.3 Formas de Interacão . . . . . . . . . . . . . . . . . . . . . . . 66

5.3 .1 Modelo $Q=A_{v, \rho_{2}}+A_{m} \rho_{m} \ldots \ldots \ldots \ldots$. . . . . . . . 66

5.3 .2 Modelo de Wang e Meng . . . . . . . . . . . . . . . . . . . . . . . 68

5.3 .3 Modificacões na Densidade da matéria . . . . . . . . . . . . . 68

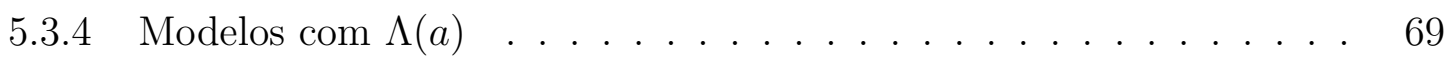

6. Tensão SNe-CMB e o Decaimento do Vácud . . . . . . . . . . . . . . . . . . . . 71

6.1 Histórico . . . . . . . . . . . . . . . . . . . . . . . 71

6.1.1 Modelo de Ozer e Taha . . . . . . . . . . . . . . . . . . . . 71

6.1 .2 Modelo de Chen e Wu . . . . . . . . . . . . . . . . . . . 73

6.1.3 Modelo de Abdel-Rahman . . . . . . . . . . . . . . . . 74 
6.1.4 Modelo de Carvalho. Lima e Waga . . . . . . . . . . . . . . 75

6.1 .5 Modelo de Lopez e Nanopoulos ... . . . . . . . . . . . . . . . 75

6.1.6 Modelo de Chen e $\mathrm{Wu}$ Generalizado . . . . . . . . . . . . . . . . 75

6.1.7 Análise Termodinâmica . . . . . . . . . . . . . . . . . . . . 76

6.2 Motivacões . . . . . . . . . . . . . . . . . . . . . . . . . . . . 77

6.2.1 Termo Constante . . . . . . . . . . . . . . . . . . . . . . . . . 77

6.2 .2 Tensão SNe-CMB . . . . . . . . . . . . . . . . . . . . . 77

6.3 Novo Modelo: $\Lambda_{0}+3 \alpha a^{-2} \ldots \ldots \ldots \ldots \ldots \ldots . \ldots \ldots$

6.3.1 Densidade da Matéria Escura e Parâmetro de Hubble . . . . . . . . 79

6.3.2 Parâmetros de Densidade de Desaceleração . . . . . . . . . . . . . 82

6.3 .3 Caso Plano . . . . . . . . . . . . . . . . . . 85

6.3 .4 Idade no Modelo Pland . . . . . . . . . . . . . . . . . . . . . . 85

6.4 Testes Observacionais . . . . . . . . . . . . . . . . . . . . . . . . . . 86

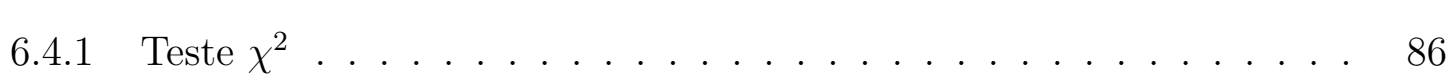

6.4.2 Contornos de Confiança e Melhor Ajuste . . . . . . . . . . . . . 89

7. Conclusões e Perspectivas . . . . . . . . . . . . . . . . . . . . . . . . . . 91

Referências . . . . . . . . . . . . . . . . . . . . . . . 95 

Capítulo 1

\section{Introdução}

\subsection{Apresentação}

Um dos grandes desafios atuais da cosmologia bem como da física em geral é determinar a natureza da energia escura, uma forma de energia proposta para explicar a aceleração cósmica descoberta em 1998 (Riess et al., 1998; Perlmutter et al., 1999). O paradigma cosmológico atual diz que cerca de $70 \%$ da energia do universo esta nesta forma, $27 \%$ na forma de matéria escura e o restante é composto pela componente bariônica, que envolve matéria ordinária e radiação.

Estas conclusões sobre a composição do universo veio com as observações resultantes da construção de grandes telescópios e o trabalho de inúmeros investigadores que conseguiram realizar o que seria impensável até duas décadas atrás. Os principais resultados estão associados com três conjuntos de dados provenientes das observações de supernovas distantes (SNe, Suzuki et al. 2012), da observação da radiação cósmica de fundo (Cosmic Microwave Background, CMB, Hinshaw et al. 2013; Ade et al. 2013) que permeia todo o universo e da observação de picos acústicos possível graças a estatística em grandes mapeamentos de galáxias (BAO, Eisenstein et al. 2005).

Energia escura é a denominação geral para a componente cósmica que causa a aceleração. Embora a densidade de energia do vácuo seja atualmente o melhor candidato, existem inúmeros modelos que desempenham este papel. No presente trabalho estudaremos uma classe destes modelos em que há interação no setor escuro, ou seja, entre matéria e energia escura. Mais especificamente, vamos focar num modelo em que haja decaimento do vácuo em matéria escura ou vice-versa. As motivações principais para este trabalho são: 
1. Modelos com decaimento do vácuo $(\Lambda(t))$ podem lançar uma luz sobre os problemas da constante cosmológica (PCC) e também sobre o chamado problema da coincidência cósmica.

2. O modelo em particular estudado pode aliviar a tensão entre os resultados dos testes observacionais de SNe e CMB.

O PCC se trata da enorme diferença entre o valor da densidade de energia do vácuo $\left(\rho_{v}=\frac{\Lambda}{8 \pi G}\right)$ observacionalmente medido e o calculado através de teoria quântica de campos (Weinberg, 1989). O problema da coincidência procura saber porque as densidades de energia da matéria e energia escura possuem a mesma ordem de grandeza no momento cosmológico atual, pois, de acordo com o modelo padrão, estas densidades evoluem de maneira muito diferente com o tempo, logo hoje seria uma época especial da vida do universo. Na segunda motivação nos referimos as diferenças nos dados de SNe e CMB, que durante o trabalhos chamaremos de tensão SNe-CMB.

\subsection{Organização do Trabalho}

No capítulo 2 faremos uma revisão geral da cosmologia de Friedmann-RobertsonWalker, na seção 2.2 discutiremos a métrica de FRW, as equações dinâmicas e os parâmetros cosmológicos. Na seção 2.3 apresentaremos as componentes que constituem o universo, com isso pode-se discutir alguns modelos cosmológicos clássicos, já na seção 2.4.

As várias distâncias cosmológicas estão definidas no capítulo 3. Estas definições serão utilizadas na seção que trata das principais observações (3.2). Os testes observacionais apresentados são SNe, CMB e BAO.

No capítulo 4, trataremos do atual modelo padrão da cosmologia $(\Lambda C D M)$ e outros candidatos para energia escura. Começando com o modelo padrão e os problemas associados com a constante cosmológica, fecharemos o capítulo com algumas propostas para a energia escura.

No capítulo 5 discutiremos, de maneira geral, a interação entre energia e matéria escura. Motivado pelos problemas apresentados no capítulo anterior, algumas formas de interação serão mostradas como exemplos.

Finalmente, no capítulo 6, discutiremos a tensão SNe-CMB a luz de nosso modelo com decaimento do vácuo. Faremos um estudo detalhado de suas previsões e aplicaremos 
os testes observacionais discutidos em 3.2 a fim de obter os melhores ajustes para os parâmetros do modelo. No capítulo 7, apresentamos as conclusões e perspectivas do nosso trabalho.

A parte original do presente trabalho se encontra nas seções 6.2, 6.3 e 6.4.

\subsection{Notações}

Neste trabalho a métrica terá assinatura $(+,-,-,-)$ e o sistema de unidades usado será o natural, com $c=\hbar=1$, exceto quando quisermos explicitá-las. Ao citar valores numéricos usaremos unidades típicas da astronomia (e.g. pc, Gyr), ou o sistema internacional de unidades. 
Capítulo 2

\section{Cosmologias Do Tipo FRW}

Neste capítulo apresentaremos uma breve revisão dos fundamentos da cosmologia moderna. Concentraremos nossa atenção nos chamados modelos do tipo Friedmann-RobertsonWalker (FRW).

\subsection{Equações de Einstein}

Em 1916 Einstein apresentou a teoria da relatividade geral, sendo possível pela primeira vez na história o estudo da cosmologia 1 . O primeiro modelo cosmológico foi feito pelo próprio Einstein. O universo de Einstein (como ficou conhecido o modelo) é estático e fechado, refletindo o desconhecimento da época a respeito da distribuição de matéria do universo e também do seu estado de expansão. Mais adiante discutiremos com detalhe este e outros modelos cosmológicos, nesta seção o foco será a relatividade geral, publicada em 1915 (Einstein, 1915, 1916).

A relatividade geral é uma extensão da relatividade restrita para descrever a interação gravitacional. O ponto de partida foi o chamado princípio da equivalência entre gravitação e inércia, proposto por Einstein em 1907 (Weinberg, 1972). Tal princípio nada mais é que uma generalização do fato de que por causa da equivalência entre as massas gravitacionais e inerciais, um observador em queda livre não sente os efeitos da gravitação. O princípio diz que para todo espaço-tempo gerado por um campo gravitacional arbitrário, existe um referêncial inercial local no qual os efeitos de gravitação estão ausentes (Weinberg, 1972;

\footnotetext{
${ }^{1}$ Apesar de ter sido desenvolvida posteriormente, é possível estudar cosmologia com base na teoria newtoniana da gravitação. O artigo pioneiro é de McCrea e Milne (1934), estando a teoria descrita em detalhes em Bondi (1968), Cap. IX. Uma generalização Neo-Newtoniana foi posteriormente proposta por Lima et al. (1997).
} 
Peebles, 1993).

A relatividade geral é uma teoria sobre a geometria do espaço-tempo, então geometrias não euclidianas são de extrema importância As equações de Einstein da relatividade geral são (Weinberg, 1972):

$$
G^{\mu \nu}=8 \pi G T^{\mu \nu}
$$

onde $G^{\mu \nu}=R^{\mu \nu}-\frac{R}{2} g^{\mu \nu}$ é o tensor de Einstein e $T_{\mu \nu}$ é o tensor energia-momento. O lado esquerdo de (2.1) representa a geometria do espaço tempo, o lado direito o conteúdo material. Tais equações dizem como as formas de energia curvam o espaço-tempo.

Para resolver estas equações é preciso fazer algumas hipóteses sobre a geometria. Por exemplo, para um espaço-tempo com simetria esférica e toda massa concentrada na origem obtemos a solução de Schwarzschild. Para aplicações cosmológicas Einstein indroduziu o chamado princípio cosmológico.

\subsection{Métrica de FRW e Equações de Friedmann}

O princípio cosmológico é uma generalização do princípio copernicano e basicamente se traduz nos conceitos de homogeneidade e isotropia2, ou seja, todos os pontos e direções do espaço são equivalentes. Com base nisso pode-se construir uma métrica, conhecida como métrica de Friedmann-Robertson-Walker (Robertson, 1935; Friedmann, 1922, 1924; Walker, 1936):

$$
d s^{2}=d t^{2}-a^{2}(t)\left[\frac{d r^{2}}{1-\kappa r^{2}}+r^{2}\left(d \theta^{2}+\sin ^{2} \theta d \phi^{2}\right)\right],
$$

onde $t, r, \theta$ e $\phi$ são as coordenadas espaço-temporais, $\kappa(=0, \pm 1)$ é o parâmetro de curvatura espacial e $a(t)$ é uma função arbitrária do tempo a ser determinada pelas equações de Einstein. O parâmetro de curvatura pode assumir três valores distintos: $0,-1$ ou 1 . Quando $\kappa=0$ o universo é dito plano, ou seja, sua geometria espacial é plana. No caso

\footnotetext{
${ }^{2} \mathrm{O}$ princípio copernicano diz que a Terra não ocupa uma posição privilegiada no universo. O termo princípio copernicano foi cunhado por Bondi (1968). Posteriormente ele também propôs o princípio cosmológico perfeito, pelo qual o universo é homogêneo e isotrópico no espaço e no tempo. Tal princípio conduziu a chamada cosmologia de estado estacionário.
} 
$\kappa=-1$ a geometria é aberta, analogamente à uma sela, o último caso, com $\kappa=1$, o universo é fechado, em duas dimensões a analogia é a superfície de uma esfera.

O tensor energia-momento que aparece do lado direito das equações de Einstein em geral toma a forma de um fluido perfeito, a saber (Dodelson, 2003)

$$
T^{\mu \nu}=(p+\rho) u^{\mu} u_{\nu}-p g^{\mu \nu}
$$

onde $p$ é a pressão, $\rho$ é a densidade de energia e $u_{\alpha}$ é a quadrivelocidade do fluido.

Para um observador comóvel $\left(u^{\mu}=\delta_{0}^{\mu}\right)$, das equações (2.1), (2.2) e (2.3) obtemos as chamadas equações de Friedmann (Weinberg, 1972):

$$
\left(\frac{\dot{a}}{a}\right)^{2}=\frac{8 \pi G}{3} \rho-\frac{\kappa}{a^{2}},
$$

e

$$
\frac{\ddot{a}}{a}=-\frac{4 \pi G}{3}(\rho+3 p) .
$$

A lei de conservação de energia $\left(u_{\mu} T_{; \nu}^{\mu \nu}=0\right)$ toma a seguinte forma:

$$
\dot{\rho}+3 \frac{\dot{a}}{a}(p+\rho)=0
$$

e pode também ser deduzida diretamente das equações de Friedmann acima. Para prosseguir é preciso determinar quem são os ingredientes que compõe o universo. As diversas componentes materiais e energéticas preenchendo o cosmos são usualmente parametrizadas através de uma equação de estado ( $\omega$-law):

$$
p=\omega \rho,
$$

onde $\omega$ é uma constante conhecida como parâmetro da equação de estado. Substituindo (2.7) em (2.6) pode-se obter a densidade de energia em função do fator de escala:

$$
\rho \propto a^{-3(1+\omega)}
$$

Para $\omega=0,1 / 3$ ou -1 , temos, respectivamente, matéria $\left(\rho_{m} \propto a^{-3}\right)$, radiação $\left(\rho_{r} \propto\right.$ $\left.a^{-4}\right)$ e vácuo ( $\rho_{v}$ constante).

A curvatura é determinada pelas densidades de energia das diversas componentes comparadas com uma densidade crítica: 


$$
\rho_{\text {crit }}=\frac{3 H^{2}}{8 \pi G},
$$

caso a densidade total seja menor que a densidade crítica o universo é aberto, caso seja maior o universo é fechado, quando for exatamente igual o universo é plano.

$\mathrm{Na}$ descrição da dinâmica cosmológica, alguns parâmetros desempenham um papel especial. O primeiro deles é o próprio parâmetro de Hubble, que aparece diretamente nas equações de Friedmann:

$$
H=\frac{\dot{a}}{a},
$$

outros parâmetros importantes são os chamados parâmetros de densidade, definidos como:

$$
\Omega_{i}=\frac{8 \pi G}{3 H^{2}} \rho_{i}=\frac{\rho_{i}}{\rho_{\text {crit }}},
$$

onde utilizamos a definição da densidade crítica dada por (2.9) , ou seja, o parâmetro de densidade nada mais é que a razão entre a densidade de uma certa componente " $i$ " em relação à densidade crítica. De maneira similar podemos definir a curvatura em termos dos parâmetros de densidade:

$$
\Omega_{\text {total }}=\sum_{i} \Omega_{i}=1+\frac{\kappa}{a^{2} H^{2}} .
$$

Introduzindo uma parâmetro de curvatura:

$$
\Omega_{\kappa}=-\frac{\kappa}{a^{2} H^{2}},
$$

podemos reescrever a primeira equação de Friedman na seguinte forma:

$$
\sum_{i} \Omega_{i}+\Omega_{\kappa}=1
$$

Com isso podemos ver porque a densidade crítica determina a curvatura, se a soma da densidade de todas as componentes for igual à densidade crítica, vemos que a somatória de (2.14) são iguais a um, ou seja, $\kappa$ deve ser zero, caso a soma seja menor $\kappa$ deve ser negativo (universo hiperbólico), caso seja maior $\kappa$ será positivo (universo fechado).

Outro parâmetro cosmológico importante é o chamado parâmetro de desaceleração: 


$$
q=-\frac{a \ddot{a}}{\dot{a}^{2}}
$$

o sinal menos na expressão acima foi introduzido pois na época acreditava-se que o universo deveria expandir desaceleradamente, já que a gravidade é atrativa. O parâmetro de Hubble está relacionado com a primeira derivada do fator de escala, o parâmetro de desaceleração com a segunda, existem outros parâmetros associados com as derivadas de ordem superior, porém são raramente utilizados na cosmologia (Turner e Riess, 2002; Visser, 2004; Guimarães et al., 2009).

Uma prática comum em cosmologia é usar o índice 0 para se referir ao tempo atual, pois é natural usar as quantidades hoje como constantes de integração.

Em cosmologia raramente se usa o tempo como variável, em seu lugar costuma-se usar o próprio fator de escala ou o redshift (desvio para o vermelho, em português), cujo símbolo é z. O redshift é o desvio para comprimentos de onda maiores que é observadd 3 em objetos distantes devido a expansão cósmica, sendo diretamente associado ao fator de escala. A definição de redshift é:

$$
z=\frac{\lambda_{e m i t}-\lambda_{o b s}}{\lambda_{o b s}}=\frac{\Delta \lambda}{\lambda},
$$

onde $\lambda_{\text {emit }}$ é a frequência da luz emitida e $\lambda_{\text {obs }}$ a da luz observada. Para estabelecer a conexão com o fator de escala considera-se um raio de luz viajando até o observador por uma direção radial, que segue um elemento de linha nulo, então usando a métrica de FRW (2.2):

$$
d t=-a(t) \frac{d r}{\sqrt{1-\kappa r^{2}}},
$$

com o sinal de menos porque a luz vem em direção a origem. Considerando a fonte com coordenada $r_{1}$ e o instante em que a luz é emitida $t_{1}$ segue que:

$$
\int_{t_{1}}^{t_{0}} \frac{d t}{a(t)}=\int_{0}^{r_{1}} \frac{d r}{\sqrt{1-\kappa r^{2}}}
$$

\footnotetext{
${ }^{3}$ Existe também o o desvio para o azul (blueshift), que é o desvio para comprimentos de onda menores, que ocorre para objetos que estão se aproximando de nós. Um exemplo de galáxia que tem blueshift é Andromeda. Tal fenômeno ocorre apenas no cosmo local, não sendo importante para a descrição do universo em larga escala.
} 
agora considerando um fóton emitido em $t_{1}+\delta t_{1}$ e detectado em $t_{0}+\delta t_{0}$ :

$$
\int_{t_{1}+\delta t_{1}}^{t_{0}+\delta t_{0}} \frac{d t}{a(t)}=\int_{0}^{r_{1}} \frac{d r}{\sqrt{1-\kappa r^{2}}},
$$

o lado direito da equação (2.19) é o mesmo que em (2.18) porque a coordenada radial de um objeto comóvel é constante. Igualando as duas expressões e rearranjando as integrais temos:

$$
\int_{t_{0}}^{t_{0}+\delta t_{0}} \frac{d t}{a(t)}=\int_{t_{1}}^{t_{1}+\delta t_{1}} \frac{d t}{a(t)},
$$

diferenciando a expressão acima e considerando que o fator de escala não varia apreciavelmente nos intervalos de tempo entre as emissões (ou recepções) sucessivas dos fótons obtém-se:

$$
\frac{\delta t_{1}}{a\left(t_{1}\right)}=\frac{\delta t_{0}}{a_{0}}
$$

Se este intervalo de tempo corresponder ao comprimento de onda da radiação temos que:

$$
\frac{\lambda_{1}}{\lambda_{0}}=\frac{a\left(t_{1}\right)}{a_{0}},
$$

utilizando (2.16) percebe-se que o lado esquerdo da equação $(2.22)$ é $\frac{1}{1+z}$, então:

$$
a(t)=\frac{a_{0}}{1+z} .
$$

Por fim a idade do universo em dado redshift $z$ pode ser calculada pela definição do parâmetro de Hubble (2.10), isolando $d t$ e integrando:

$$
t(z)=\frac{1}{H_{0}} \int_{0}^{1 / 1+z} \frac{d a}{a E(a)},
$$

onde $E=H / H_{0}$. Para calcular a idade hoje basta supor $z=0$, ou seja, $a=a_{0}$. Temos:

$$
H_{0} t_{0}=\int_{0}^{1} \frac{d x}{x E(x)}
$$

onde $x=a / a_{0}$. A partir da expressão de $E(a)$ obtemos de (2.24) e (2.25) as idades $t(z)$ ou $t_{0}$ em qualquer modelo cosmológico baseado na métrica do tipo FRW. 


\subsection{Componentes do Universo}

As principais componentes que podem constituir o universo estão descritas a seguir.

\subsubsection{Matéria Bariônica}

A componente mais óbvia para qualquer modelo cosmológico é a matéria ordinária, que localmente constitui estrelas e planetas, normalmente chamada de matéria bariônicat 4 .

A equação de estado da componente cósmica material é $p=0$, ou seja, esta componente não contribui com pressão. Para entender o motivo desta equação de estado basta lembrar a pressão de um gás é dada por $p=\frac{n m \overline{v^{2}}}{3}$, onde $n$ é a densidade numérica, $m$ é a massa e $\overline{v^{2}}$ é a média da velocidade ao quadrado. O "gás" tratado em cosmologia é formado pelas galáxias e aglomerados, que possuem velocidade peculiar muito pequena (em comparação com a velocidade da luz). Assim, ao se restaurar o fator $c$ nas equações de Friedmann vemos que a contribuição da pressão é desprezível.

O valor do parâmetro de densidade dos bárions é obtido com dados de CMB, BAO e aglomerados e vale cerca de 0,045 .

\subsubsection{Radiação}

Outra componente óbvia são os fótons que permeiam o universo. Como será discutido adiante, a maior contribuição radiativa está associada a radiação cósmica de fundo, na região de microondas.

Qualquer partícula ultrarelativística (cuja energia cinética é muito maior que sua massa) também contribui como radiação, pois para $K_{b} T>>m c^{2}$ a equação de estado tende para $p=\frac{1}{3} \rho($ Groot et al., 1980) $)$.

Conforme veremos a maior contribuição para a densidade de energia dos fótons vem da radiação cósmica de fundo, que possui uma temperatura extremamente bem determinada de $2,725 K$. A partir desse é fácil ver que a contribuição para o parâmetro de densidade total é de apenas $\Omega_{r} \approx 10^{-5}$ e portanto bem menor do que da matéria (escura ou bariônica).

\footnotetext{
${ }^{4} \mathrm{O}$ termo bárion, no contexto de física de partículas, remete ao estado ligado de três quarks, cujos exemplos mais comum são os prótons e nêutrons. Esta denominação é usada porque praticamente toda a massa dos átomos está associada aos núcleos, ou seja, a contribuição dos elétrons (que são léptons) é desprezível.
} 


\subsubsection{Matéria Escura}

Matéria escura é a denominação para qualquer tipo de matéria que não interage com a luz. De maneira geral, a matéria escura é classificada em quente, fria ou morna (HDM, CDM e WDM, respectivamente, nas siglas em inglês), a diferença é que a fria é não relativística e a quente é ultrarelatívistica, sendo morna no caso intermediário. Atualmente acredita-se que a matéria escura é fria, então sua equação de estado é a mesma da matéria bariônica: $p=0$.

As primeiras observações que levaram à matéria escura foram feitas por F. Zwicky na década de 30 (Zwicky, 1933); ao estudar a velocidade de dispersão de galáxias em aglomerados ele percebeu que deveria existir muito mais massa nesses sistemas do que era estimado através da emissão de luz (massa bariônica).

As observações que definitivamente convenceram a comunidade astronômica da existência da matéria escura foram feitas por V. Rubin já na década de 1970 . Ela mediu a curva de rotação de várias galáxias e concluiu que deveria exister mais massa nesse sistema do que era observado (Rubin e Ford, 1970; Rubin et al., 1985).

A rotação das galáxias espirais segue a lei de Newton:

$$
v_{r o t}=\left(\frac{G M}{r}\right)^{1 / 2},
$$

onde $r$ é a distância ao centro e $M$ é a massa interior ao raio $r$. Longe do centro, na região onde há pouca matéria visível esperamos que a velocidade de rotação tenha a forma $r^{-1 / 2}$ pois toda a massa do sistema esta dentro do raio. No entanto V. Rubin mediu que essas velocidades se mantêm aproximadamente constantes, logo deve haver grande quantidade de massa que não emite luz nas regiões externas das galáxias. Esta matéria compõe os chamados halos de matéria escura de galáxias e aglomerados.

\subsubsection{Vácuo}

Antigamente entendia-se por vácuo a completa ausência de matéria ou qualquer forma de energia. Porém, com a advento da teoria quântica de campos essa concepção foi modificada. Agora se define o vácuo como sendo o estado de mais baixa energia de um campo quântico e, portanto, o vácuo carrega energia.

A energia do vácuo resultante de todos os campos quânticos existentes no universo 
pode ser identificada com a chamada constante cosmológica, dada por:

$$
\rho_{v}=\frac{\Lambda}{8 \pi G},
$$

a constante cosmológica tem uma história interessante. Inicialmente este termo foi introduzido por Einstein (1917) nas equações de campo (2.1) para viabilizar seu modelo cosmológico estático (o modelo de Einstein será discutido mais a frente, na seção 2.4.1). Naquela época não se sabia que o vácuo carregava energia, então a constante cosmológica foi introduzida como um termo geométrico, ou seja, do lado esquerdo das equações. Nesta interpretação geométrica $\Lambda$ aparece como uma constante de integração, normalmente tida como zero no desenvolvimento da relatividade geral padrão.

Posteriormente, quando Hubble descobriu a expansão do universo, Einstein descartou a constante cosmológica pois a considerou desnecessária (Einstein, 1931), por outro lado, as estimativas da constante de Hubble da época emplicavam em uma idade do universo muito baixa comparada com algumas estimativas da idade da Via Láctea. Lemaitre argumentou que a constante cosmológica aumentava a idade do universo e poderia resolver o problema da idade. Assim $\Lambda$ nunca foi totalmente abandonada5.

As evidências para a constante cosmológica até meados dos anos 90 eram: A identificação feita de que a energia do vácuo era efetivamente uma constante cosmológica; as baixas medidas da densidade de energia da matéria que não eram o suficiente para um universo plano, conforme indicava as observações da radiação cósmica de fundo e as teorias inflacionárias; e o problema da idade, que nunca foi totalmente resolvido mesmo quando as medidas da constante de Hubble cairam para cerca de $10 \%$ das estimativas originais. A evidência mais confiável surgiu em 1998 quando dois grupos independentes reportaram a expansão acelerada do universo (Riess et al., 1998; Perlmutter et al., 1999).

Como foi visto anteriormente $\Lambda$ pode ser interpretada como a densidade de energia do vácuo. Pensando o vácuo como um fluido perfeito é fácil verificar que sua equação de estado é dada por:

$$
p_{v}=-\rho_{v}
$$

para obter esta relação notamos que as propriedades do vácuo são invariantes de Lorentz,

\footnotetext{
${ }^{5}$ Para uma história da constante cosmológica veja Straumann (2002); Demiański (2000); Lima (2004).
} 
ou seja, transformações de Lorentz arbitrárias não modificam a forma do tensor de energia momento (Grøn, 1986; Cunha, 2002) e portanto, o TEM deve se transformar como:

$$
T_{\mu \nu}=T_{\mu^{\prime} \nu^{\prime}}=L_{\mu^{\prime}}^{\alpha} L_{\nu^{\prime}}^{\beta} T_{\alpha \beta},
$$

primeiramente, considerando um boost de volocidade $v$ na direção $\widehat{x}$, obtém-se:

$$
T_{00}=T_{0^{\prime} 0^{\prime}}=\gamma^{2}\left[T_{00}+v\left(T_{01}+T_{10}+v T_{11}\right)\right],
$$

onde $\gamma=\left(1-v^{2}\right)^{1 / 2}$ é o fator de Lorentz. Isolando $v$ lembrando que:

$$
v=T_{00}+T_{11}+T_{01}+T_{10}
$$

a transformação da componente 11 resulta na mesma equação. Transformando as componentes $T_{01}$ e $T_{10}$ temos:

$$
T_{00}+T_{11}+v\left(T_{01}+T_{10}\right)=0
$$

obtendo:

$$
T_{11}=-T_{00},
$$

e

$$
T_{01}=-T_{10}
$$

As transformações de $T_{02}$ e $T_{12}$ resultam em:

$$
T_{02}=\gamma T_{02}+\gamma v T_{12},
$$

e

$$
T_{12}=\gamma v T_{02}+\gamma T_{12},
$$

o que implica que $T_{02}=T_{12}=0$. Da mesma forma é possível determinar que:

$$
T_{20}=T_{21}=T_{03}=T_{13}=T_{30}=T_{31}=0 .
$$

Agora considerando um boost na direção $\widehat{y}$ obtém-se: 


$$
T_{01}=T_{10}=T_{23}=T_{32}=0,
$$

e

$$
T_{22}=-T_{00}
$$

E por fim um boost na direção $\widehat{z}$ leva a:

$$
T_{33}=-T_{00}
$$

Juntando todas estas informações:

$$
T_{\mu \nu}=T_{00} \operatorname{diag}(1,-1,-1,-1),
$$

ou seja:

$$
T_{\mu \nu}=T_{00} \eta_{\mu \nu}
$$

lembrando que a interpretação de $T_{00}$ é a densidade de energia da componente considerada, neste caso o vácuo. Para uma métrica arbitrária basta substituir $\eta_{\mu \nu}$ por $g_{\mu \nu}$ :

$$
T_{\mu \nu}=\rho_{v} g_{\mu \nu},
$$

comparando com a expressão de um fluido perfeito (2.3) chega-se a relação (2.28).

De posse destes ingredientes podemos discutir os vários modelos cosmológicos, o que será feito na seção 2.4 .

\subsection{Modelos Cosmológicos}

\subsubsection{Modelo de Einstein}

O modelo de Einstein foi o primeiro modelo cosmológico, proposto em 1917 (Einstein, 1917), um ano após a publicação da relatividade geral. Naquela época ainda não se tinha uma visão clara do que era uma galáxia e ainda não existiam as observações de Hubble que determinaram a expansão universal, então a visão predominante é que o universo era estático, pois essa era a impressão que o céu noturno passava. Einstein então construiu seu 
modelo para ser estático, ou seja, a função $a(t)$ que aparece nas equações de Friedmann (2.4) e (2.5) é constante.

Para o contéudo material Einstein supôs que existia apenas matéria pois era apenas isso que observavam na época, ou seja, podemos eliminar o termo de pressão em (2.5). As equações resultantes são:

$$
\frac{8 \pi G}{3} \rho+\frac{\Lambda}{3}-\frac{\kappa}{a^{2}}=0
$$

e

$$
\frac{4 \pi G}{3} \rho=\frac{\Lambda}{3},
$$

onde se deixou explícita a constante cosmológica. Eliminando $\rho$ da primeira equação obtém-se uma expressão para $\Lambda$ :

$$
\Lambda=\frac{\kappa}{a^{2}},
$$

da segunda equação obtemos a densidade em função da constante cosmológica:

$$
\rho=\frac{\Lambda}{4 \pi G}=\frac{\kappa}{4 \pi G a^{2}},
$$

como a densidade deve ser positiva implica que $\kappa=1$.

\subsubsection{Modelo de De Sitter}

Neste modelo, proposto por de Sitter (1917), não há presença de matéria, radiação ou curvatura, somente constante cosmológica. Eliminando a densidade, a pressão e a curvatura das equações de Friedmann:

$$
\dot{a}=\sqrt{\frac{\Lambda}{3}} a
$$

integrando:

$$
a(t)=a_{0} \exp \sqrt{\frac{\Lambda}{3}}\left(t-t_{0}\right),
$$

neste modelo o parâmetro de Hubble é constante e vale $\frac{\Lambda}{3}$. 


\subsubsection{Modelo de Einstein-De Sitter}

Este modelo, proposto por Einstein e de Sitter (1932), é semelhante ao modelo de Einstein pois apenas a matéria domina, não há constante cosmológica e o universo é plano. Usando a expressão (2.8) $\operatorname{com} \omega=0$ para obter a densidade em função do fator de escala e substituindo na primeira equação de Friedmann:

$$
\left(\frac{\dot{a}}{a}\right)^{2} \propto \frac{8 \pi G}{3} a^{-3},
$$

resolvendo para $a$ :

$$
a(t) \propto t^{2 / 3}
$$

O parâmetro de Hubble é dado por:

$$
H(t)=\frac{2}{3 t},
$$

o que implica que a idade do universo hoje é:

$$
t_{0}=\frac{2}{3 H_{0}}
$$

Por muitos anos este foi o modelo cosmológico padrão. A componente dominante corresponde tanto a matéria bariônica quanto a matéria escura, pois possuem a mesma equação de estado. Este cenário mudou com as observações que verificaram a existência de outra componente que domina sobre a matéria, chamada de energia escura. O novo modelo, chamado de $\Lambda C D M$ será tratado no capítulo 4 .

Vários outros modelos podem ser criados ao se adicionar outras componentes, por exemplo, no começo do universo a radiação dominava sobre todas as outras componentes, então é comum resolver as equações de Friedmann com $p=\frac{1}{3} \rho$. 
Capítulo 3

\section{Distâncias e Cosmologia Observacional}

Neste capítulo discutiremos alguma expressões observacionais e resultados que serão utilizados na parte original desta dissertação (capítulo 66). Primeiramente, vamos apresentar os dois principais conceitos de distância e algumas de suas consequências para a cosmologia moderna.

\subsection{Distâncias}

Na cosmologia observacional é de fundamental importância entender o que é distância. $\mathrm{Na}$ física cotidiana este é um conceito simples, porém em escalas cosmológicas é preciso considerar que o universo está em expansão, ou seja, quando a luz de um objeto distante chega à Terra ele já não estará mais na mesma posição (embora esteja na mesma coordenada comóvel), por isso é preciso cuidado ao se definir distância em cosmologia.

Existem duas definições de distância comumente utilizadas em cosmologia: a distância de luminosidade e a de diâmetro angular. Tais distâncias são definidas com base no fluxo de energia recebido de uma vela padrão e no ângulo subtendido por uma régua padrão, respectivamente.

\subsubsection{Distância de Luminosidade}

A relação entre a luminosidade absoluta e aparente de um objeto é definida por:

$$
\ell=\frac{L}{4 \pi d^{2}}
$$

onde $\ell$ é a luminosidade aparente, $L$ é a absoluta e $d$ é a distância entre o observador e a fonte. Esta expressão é válida para objetos próximos, quando se considera grandes 
distâncias três modificações precisam ser feitas (Weinberg, 2008):

- O fator $1 / d^{2}$ deve ser modificado pois a área que passa pela Terra e tem o objeto como centro é $4 \pi r_{1}^{2} a^{2}$, onde $r_{1}$ é a coordenada do objeto visto na Terra.

- A taxa de chegada dos fótons é menor que a emitida por um fator $\frac{a\left(t_{1}\right)}{a\left(t_{0}\right)}=\frac{1}{1+z}$.

- Finalmente a energia dos fótons que chegam é menor que a energia dos fótons emitidos pelo mesmo fator de redshift $\frac{1}{1+z}$.

Considerando essas correções a expressão (3.1) toma a seguinte forma (Peebles, 1993):

$$
\ell=\frac{L}{4 \pi r_{1}^{2} a_{0}^{2}(1+z)^{2}},
$$

a distância de luminosidade é definida de tal forma que a expressão (3.2) tenha a mesma forma que (3.1):

$$
\ell=\frac{L}{4 \pi d_{L}^{2}}
$$

portanto,

$$
d_{L}=a_{0} r_{1}(1+z)
$$

Na expressão acima, a coordenada radial $r_{1}(z)$ depende do modelo. Observacionalmente se mede a luminosidade aparente, então ao se conhecer a luminosidade absoluta de algum objeto pode-se determinar sua distância. Estes objetos são chamados de velas padrão.

Para pequenos redshifts é possível achar uma expansão da distância de luminosidade que é independente do modelo. Para isto expande-se o fator de escala em uma séria de potências:

$$
a(t)=a_{0}\left[1+H_{0}\left(t-t_{0}\right)-\frac{1}{2} q_{0} H_{0}^{2}\left(t-t_{0}\right)^{2}+\ldots\right]
$$

onde se usou o parâmetro de Hubble (2.10) e o parâmetro de desaceleração (2.15). Usando a definição de redshift (2.23):

$$
z=H_{0}\left(t_{0}-t\right)+\left(1+\frac{1}{2} q_{0}\right) H_{0}^{2}\left(t_{0}-t\right)^{2}+\ldots,
$$

e invertendo a expressão acima obtemos: 


$$
t_{0}-t=\frac{1}{H_{0}}\left[z-\left(1+\frac{1}{2} q_{0}\right) z^{2}+\ldots\right] .
$$

Usando a equação (2.18) para relacionar a coordenada $r \operatorname{com} z$ :

$$
\frac{1}{a_{0}} \int_{t}^{t_{0}}\left[1+H_{0}\left(t_{0}-t\right)+\left(1+\frac{1}{2} q_{0}\right) H_{0}^{2}\left(t_{0}-t\right)^{2}+\ldots\right]=r+\mathcal{O}\left(r^{3}\right),
$$

ou seja:

$$
r=\frac{1}{a_{0}}\left[\left(t_{0}-t\right)+\frac{1}{2} H_{0}\left(t_{0}-t\right)^{1 / 2}+\ldots\right] .
$$

Substituindo (3.7) em (3.9) segue que:

$$
r=\frac{1}{a_{0} H_{0}}\left[z-\frac{1}{2}\left(1+q_{0}\right) z^{2}+\ldots\right]
$$

por fim, substituindo em (3.4) temos:

$$
d_{L}=\frac{1}{H_{0}}\left[z+\frac{1}{2}\left(1-q_{0}\right) z^{2}+\ldots\right]
$$

\subsubsection{Distância de Diâmetro Angular}

É bem conhecido que no espaço euclidiano um objeto extenso ao ser observado a uma certa distância $d_{A}$ subentende um ângulo dado por:

$$
\theta=\frac{s}{d_{A}},
$$

onde $s$ é a dimensão do objeto e $d_{A}$ é a distância até ele (o objeto é considerado perpendicular ao observador). Para grandes distâncias pode-se usar a métrica (2.2) para determinar a dimensão do objeto, obtendo $s=a_{1} r_{1} \theta$. Da mesma maneira define-se a distância de diâmetro angular de forma a preservar a relação (3.12):

$$
d_{A}=\frac{s}{\theta}=a_{1} r_{1}=\frac{a_{0} r_{1}}{1+z}
$$

então ao se conhecer a dimensão de um objeto e medir seu diâmetro angular pode-se determinar sua distância. Estes objetos são conhecidos como réguas padrão. 


\subsubsection{Relação de Etherington}

Comparando as expressões (3.4) e (3.13) obtemos a seguinte relação:

$$
\frac{d_{L}}{d_{A}}=(1+z)^{2}
$$

esta relação entre as distâncias deve ser válida para qualquer modelo cosmológico. Portanto, qualquer desvio implicaria a existência de um novo efeito físico, que pode ser tanto uma modificação na gravidade quanto efeitos astrofísicos desconhecidos. A equação (3.14) é conhecida como relação de Etherington (Etherington, 1933; Ellis, 2007; Holanda et al., 2010, 2011).

\subsubsection{Distâncias em Função dos Parâmetros Cosmológicos}

Para os testes observacionais é importante determinar como os parâmetros cosmológicos influenciam as distâncias. As expressões utilizadas nesta seção serão em função de $H(z)$, pois para cada modelo diferente o parâmetro de Hubble se modifica. Considerando a definição de $H=\frac{\dot{a}}{a}$, podemos escrever

$$
\frac{d t}{a}=\frac{d a}{a^{2} H}=\frac{d x}{x^{2} H(x)},
$$

onde $x=a / a_{0}$. Em termos do redshift $\mathrm{z}$, temos também

$$
d t=-\frac{d z}{(1+z) H(z)} .
$$

Como a luz se propaga ao longo de geodésicas nulas $\left(d s^{2}=0\right)$ sabemos que nas métricas do tipo FRW:

$$
\int_{t_{1}}^{t_{0}} \frac{d t}{a(t)}=\int_{0}^{r_{1}} \frac{d r}{\sqrt{1-\kappa r^{2}}}=\left\{\begin{array}{l}
\arcsin r \text { para } \kappa=1 \\
r \text { para } \kappa=0 \\
\operatorname{arcsinh} r \text { para } \kappa=-1
\end{array}\right.
$$

Desta forma:

$$
r(z)=\frac{1}{a_{0} H_{0} \Omega_{\kappa 0}^{1 / 2}} \sinh \left[\Omega_{\kappa 0}^{1 / 2} \int_{1 / 1+z}^{1} \frac{d x}{x^{2} E(x)}\right],
$$


onde $E(x)=H / H_{0}$. A equação (3.18) é valida para qualquer valor de $\kappa$, inclusive o caso plano $\left(\Omega_{\kappa 0}=0\right)$, que se reduz para a seguinte expressão

$$
r=\frac{1}{a_{0} H_{0}} \int_{1 / 1+z}^{1} \frac{d x}{x^{2} E(x)} .
$$

Substituindo este resultado na expressão (3.4) temos a expressão

$$
d_{L}(z)=\frac{1+z}{H_{0} \Omega_{\kappa 0}^{1 / 2}} \sinh \left[\Omega_{\kappa 0}^{1 / 2} \int_{1 / 1+z}^{1} \frac{d x}{x^{2} E(x)}\right] .
$$

\subsection{Cosmologia Observacional}

Em 1998, as observações astronômicas utilizando Supernovas do tipo Ia como velaspadrão indicaram que a expansão do universo está acelerada (Riess et al.,, 1998; Perlmutter et al., 1999), tendo a transição entre os regimes desacelerado/acelerado ocorrido em redshifts da ordem da unidade (Turner e Riess, 2002; Cunha e Lima, 2008; Cunha, 2009; Lima et al., 2012). Como a gravidade é uma força atrativa, este fato surpreendeu a comunidade de astrônomos e cosmólogos que acreditava numa expansão desacelerada. A descoberta da expansão acelerada do universo é de importância comparável a detecção da radiacão cósmica de fundo de 3K, descoberta acidentalmente em meados dos anos 60 (Penzias e Wilson, 1965). Certamente ela provoca um impacto profundo na nossa compreensão do cosmos, abre novas perspectivas para a teoria de campos, para a física de partículas elementares, e como toda grande descoberta, lança novos desafios para a comunidade científica. Nesta seção descreveremos as principais observações que nos levaram ao atual modelo cosmológico.

\subsubsection{A Velocidade de Recessão das Galáxias}

Antes de 1929 se acreditava que o universo era estático, uma concepção modificada pelo trabalho pioneiro de Hubble (1929) que, usando o telescópio de 100 polegadas (2,5 metros) do observatório de Monte Wilson, mediu a velocidade de recessão de cerca de 40 galáxias e fez um gráfico em função da distância. O gráfico original de Hubble está na figura 3.1 .

Com os dados acima, Hubble propôs uma relação linear entre a velocidade e distância das galáxias 


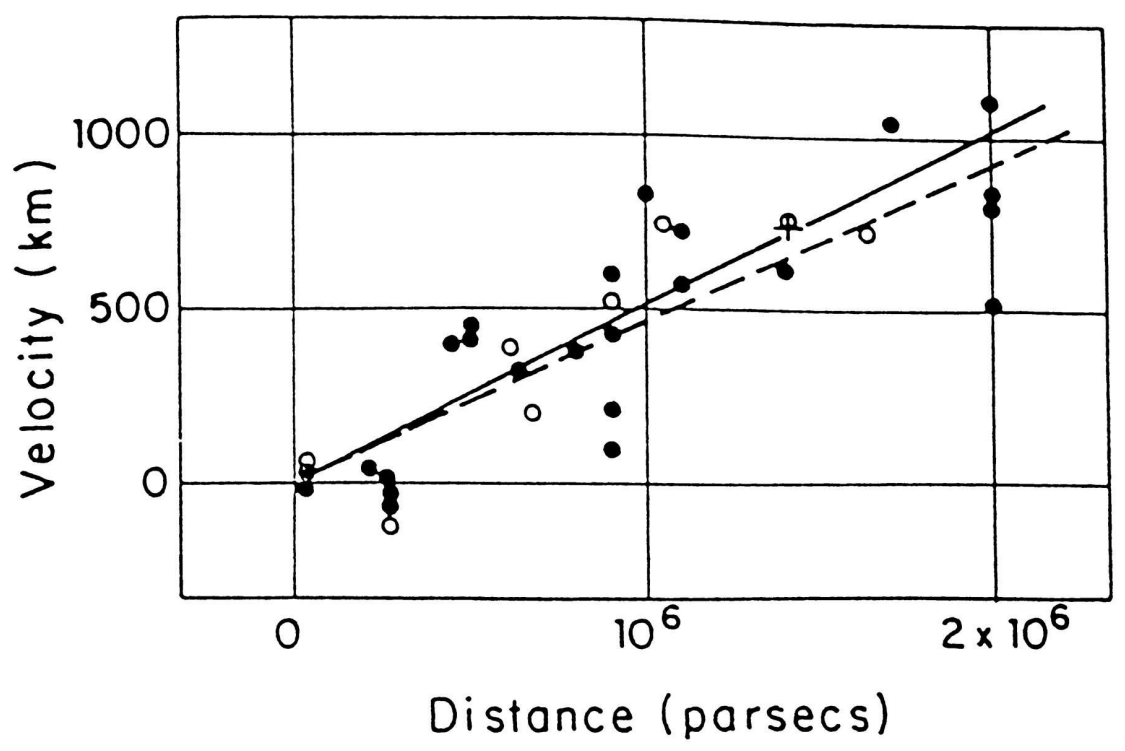

Figura 3.1: Gráfico original da relação velocidade distância obtida por Hubble (1929).

$$
v=H_{0} r
$$

onde a constante de proporcionalidade ficou conhecida como constante de Hubble. O valor originalmente determinado por Hubble foi de $500 \mathrm{~km} / \mathrm{s} / \mathrm{Mpc}$, cerca de 10 vezes maior que as estimativas modernas (Riess et al., 1998).

Para medir as distâncias de luminosidade é preciso conhecer velas padrões. Normalmente, cada vela padrão tem um intervalo de redshift em que é possível utilizá-la, sendo que para cada novas velas são calibradas usando as anteriores, na chamada escada de distâncias cósmicas. Atualmente, as supernovas do tipo Ia representam as melhores velas padrões disponíveis em cosmologia.

\subsubsection{Supernovas do Tipo Ia}

Uma supernovas é chamada de tipo I quando não apresenta linhas de hidrogênio no seu espectro, o "a" refere-se a presença de uma linha de absorção de silício ionizado. Estas SNe ocorrem em sistemas binários, quando uma anã branca acreta matéria de sua companheira até o limite de Chandrasekhar $\left(1,38 M_{\odot}\right)$, quando a anã branca torna-se instável e explode. Por causa do limite de Chandrasekhar, que é universal, há pouca variação na luminosidade dessas explosões, por isso SNe do tipo Ia são boas velas padrões.

Para serem usadas como indicadores de distância as SNe precisam ser calibradas, pois 
mesmo sendo aproximadamente velas padrões há uma dispersão de 0,4 em magnitude na luminosidade de pico. Existe uma relação empírica entre o pico de luminosidade e a forma da curva de luz, quantificada por Phillips (1993), que é usada para esta calibração.

Foi através de dados de SNe que se descobriu que o universo esta acelerado, os primeiros grupos a reportar isso foram o High-z Supernova Search Team (Riess et al., 1998) e o Supernova Cosmology Project (Perlmutter et al., 1999). Por estes trabalhos o premio nobel de 2011 foi entregue a Saul Perlmutter, Brian P. Schimidt e Adam G. Ries:11. Atualmente todos os dados de SNe estão no chamado Union2.1 compilation (Suzuki et al., 2012), que traz dados de 833 SNe, sendo que 580 destas são consideradas de qualidade para serem utilisadas nos diagramas do tipo Hubble.

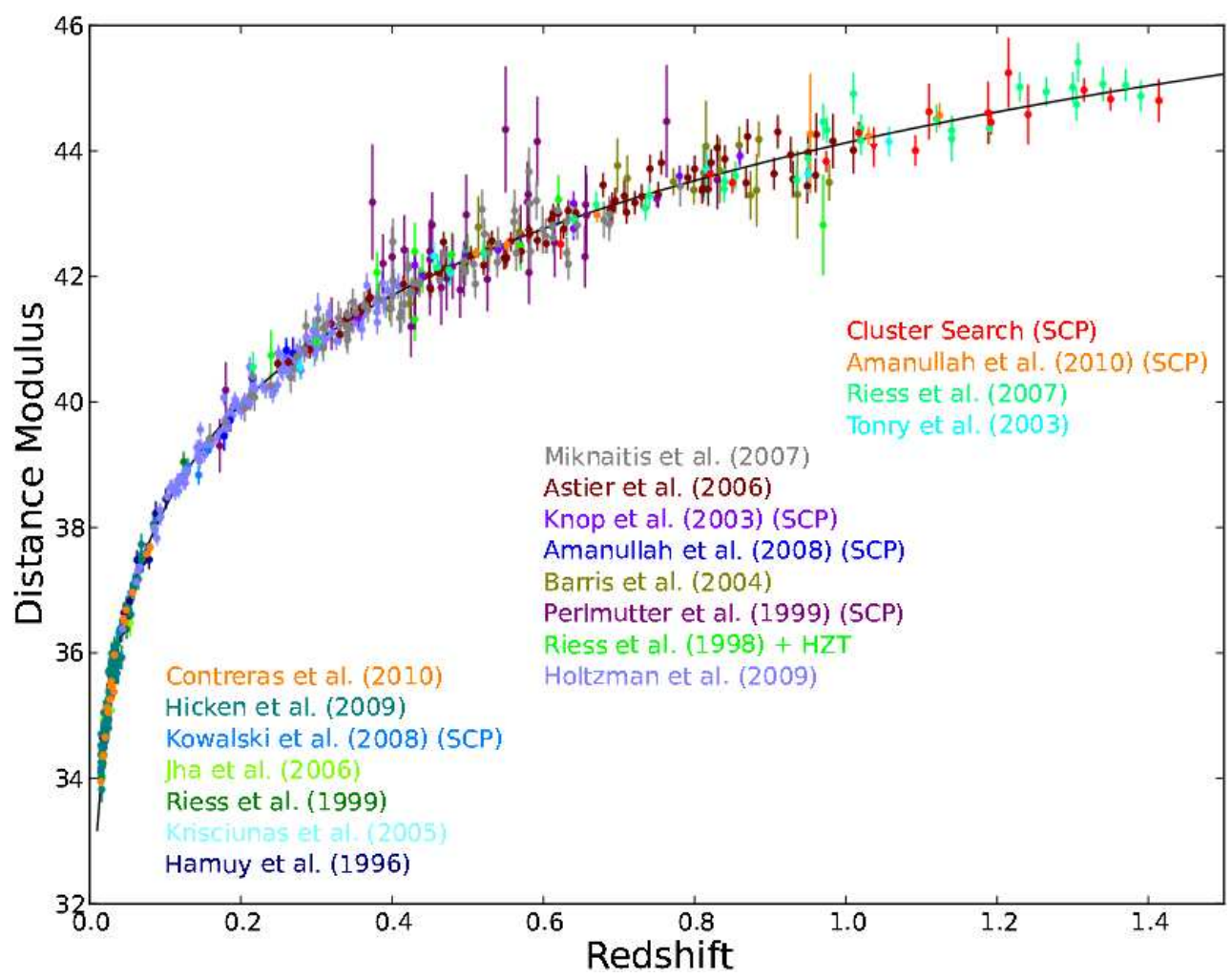

Figura 3.2: Diagrama de Hubble para SNe do tipo Ia. A figura inclui resultados das mais diversas amostras, compiladas por Suzuki et al. (2012).

Normalmente os dados de SNe são apresentados em termos do módulo de distância, definido da seguinte forma (ver figura 3.2)

$$
\mu=m-M,
$$

1 "The Nobel Prize in Physics 2011". http://www.nobelprize.org/nobel_prizes/physics/laureates/2011/ 
onde $m$ é a magnitude aparente e $M$ a absoluta do objeto. A magnitude absoluta é a magnitude que um objeto teria se estivesse a 10pc de distância, usando a equação da luminosidade (3.3) obtém-se a seguinte relação de escala (Peebles, 1993):

$$
L\left(d_{L}\right)=\frac{L(10)}{\left(\frac{d_{L}}{10}\right)^{2}}
$$

A definição de magnitude é:

$$
m=-2,5 \log _{10} L
$$

e combinando com (3.23) obtém-se o módulo de distância em função da distância de luminosidade:

$$
\mu=5 \log _{10} d_{L}-5
$$

onde $d_{L}$ é medido em $p c$. Para aplicações cosmológicas é comum utilizar $M p c$ como unidade de distância, com a relação acima tomando a seguinte forma:

$\begin{aligned} & \mu=5 \log _{10} d_{L}+25, \\ & \text { com } d_{L} \text { dado em } M p c(\text { Peebles, } 1993) .\end{aligned}$

\subsubsection{A Radiação Cósmica de Fundo}

A radiação cósmica de fundo (CMB, na sigla em inglês) é a radiação presente em todo o universo proveniente da última superfície de espalhamento (desacoplamento entre matéria bariônica e radiação, z 1100). Esta radiação foi inicialmente prevista por Alpher e Herman (1948), em estudos sobre a nucleossíntese cosmológica. Anos depois, em 1965, A. Penzias e R. Wilson mediram, usando um radiotelescópio, um excesso de $3,5 \mathrm{~K}$ de temperatura de antena no comprimento de onda de $7,5 \mathrm{~cm}$ (Penzias e Wilson, 1965). Por essa primeira medida da CMB eles receberam o premio nobel de 197\&2.

Posteriormente outros investigadores mediram a temperatura da CMB em diversas bandas de frequência, porém a sua natureza de corpo negro (figura 3.3) só foi estabelecida experimentalmente no anos 90 com o satélite COBE (COsmic Background Explorer). O projeto COBE mediu com grande precisão o espectro completo do CMB, um resultado que

\footnotetext{
2 "The Nobel Prize in Physics 1978". http://www.nobelprize.org/nobel_prizes/physics/laureates/1978/
} 
rendeu o prêmio nobel de física para seus principais investigadores, os astrofísicos George Smoot e John Mather 3 .

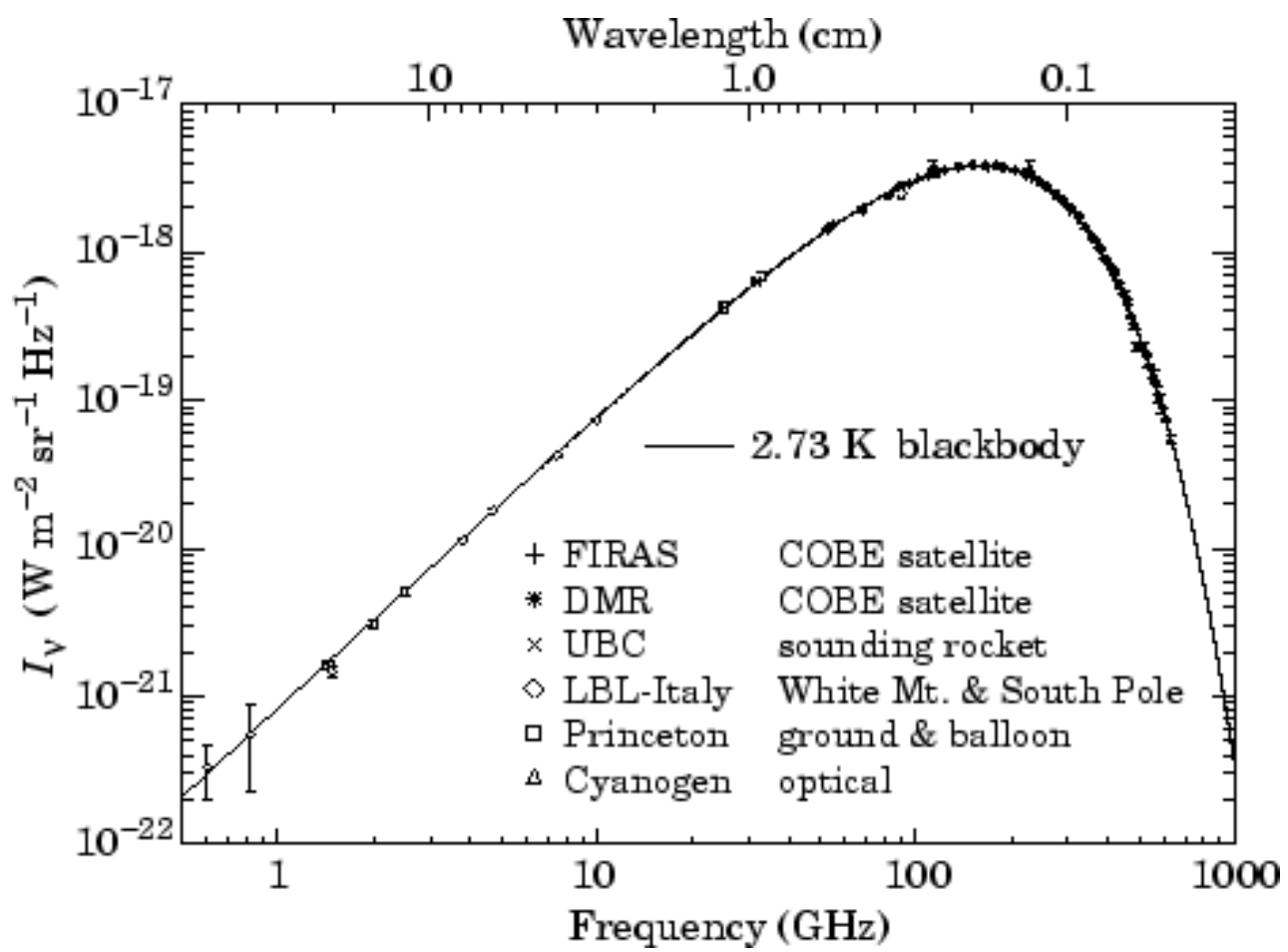

Figura 3.3: Espectro de corpo negro da radiação cósmica de fundo retirado de Smoot e Scott (1996).

Apesar do universo ser homogêneo em grande escala sabe-se que em pequenas escalas existem estruturas (galáxias, aglomerados, por exemplo), estas inomogêneidades devem se refletir na radiação cósmica de fundo. O satélite COBE foi o primeiro a medir as anisotropias de temperatura, que são de ordem $10^{-5}$, posteriormente o satélite WMAP (Wilkinson 4 Microwave Anisotropy Probe) refinou estas medidas. O gráfico 3.4 mostra as anisotropias medidas pelo WMAP.

Na última superficie de espalhamento uma anisotropia de abertura angular $\delta \theta$ tem um tamanho físico que pode ser calculado utilizando a distância de diâmetro angular (3.12). As flutuações de temperatura são definidas como (Dodelson, 2003; Pigozzo, 2010):

$$
\delta T(\theta, \phi) \equiv T(\theta, \phi)-<T>
$$

onde $\langle T>$ é a temperatura média do CMB, definida através da expressão

\footnotetext{
3 "The Nobel Prize in Physics 2006". http://www.nobelprize.org/nobel_prizes/physics/laureates/2006/

${ }^{4}$ Em homenagem à David Todd Wilkinson (1935 - 2002), que fazia parte da equipe cientifica.
} 


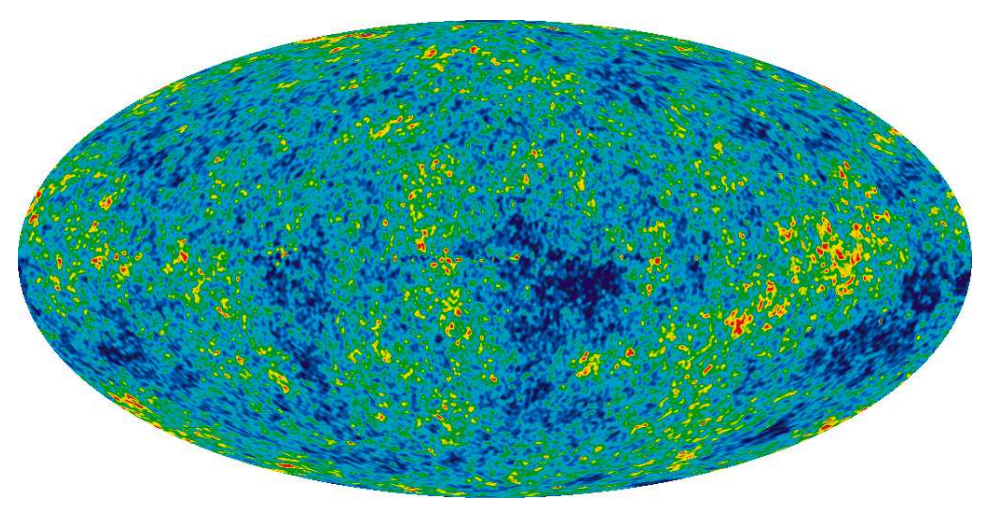

Figura 3.4: Anisotropias da radiação cósmica de fundo obtido pelo WMAP9 retirado de (http://wmap.gsfc.nasa.gov/).

$$
<T>\equiv \frac{1}{4 \pi} \int T(\theta, \phi) \sin (\theta) d \theta d \phi .
$$

Como a superfície de último espalhamente é esférica é conveniente expandir as flutuações em harmônicos esféricos

$$
\delta T(\theta, \phi)=\sum_{l m} a_{l m} Y_{l m}(\theta, \phi)
$$

onde a soma em $l$ varia de 0 até $\infty$ e a soma em $m$ vai de $-l$ até $l$. Em cosmologia o valor de apenas uma anisotropia é de pouco proveito, então seria interessante estudar a média de todas as anisotropias, porém, conforme indica as equações (3.27) e (3.28) esta média é zero. Desta forma, a quantidade não trivial mais simples para caracterizar as anisotropias é a média da correlação

$$
<\delta T(\theta, \phi) \delta T\left(\theta^{\prime}, \phi^{\prime}\right)>=\sum_{l m}<a_{l m} a_{l^{\prime} m^{\prime}}>Y_{l m} Y_{l^{\prime} m^{\prime}}
$$

onde o valor médio dos coeficientes é dado por:

$$
<a_{l m} a_{l^{\prime} m^{\prime}}>=\delta_{l l^{\prime}} \delta_{m-m^{\prime}} C_{l},
$$

substituindo (3.31) em (3.30) obtém-se (Dodelson, 2003; Weinberg, 1989):

$$
<\delta T(\theta, \phi) \delta T\left(\theta^{\prime}, \phi^{\prime}\right)>=\sum_{l} C_{l} \frac{2 l+1}{4 \pi} P_{l}(\cos \theta),
$$

com o termo $C_{l}$ relacionado a flutuação de temperatura numa dada escala angular no multiplo $l$ (grandes l's correspondem a pequenas escalas). 


$$
\theta=\frac{\pi}{l}
$$

Resumindo, quanto maior a medida de $C_{l}$ para um dado $l$ maior será as anisotropias naquela escala. A figura 3.5 mostra o gráfico de $C_{l}$ e os dados obtidos pelo WMAP.

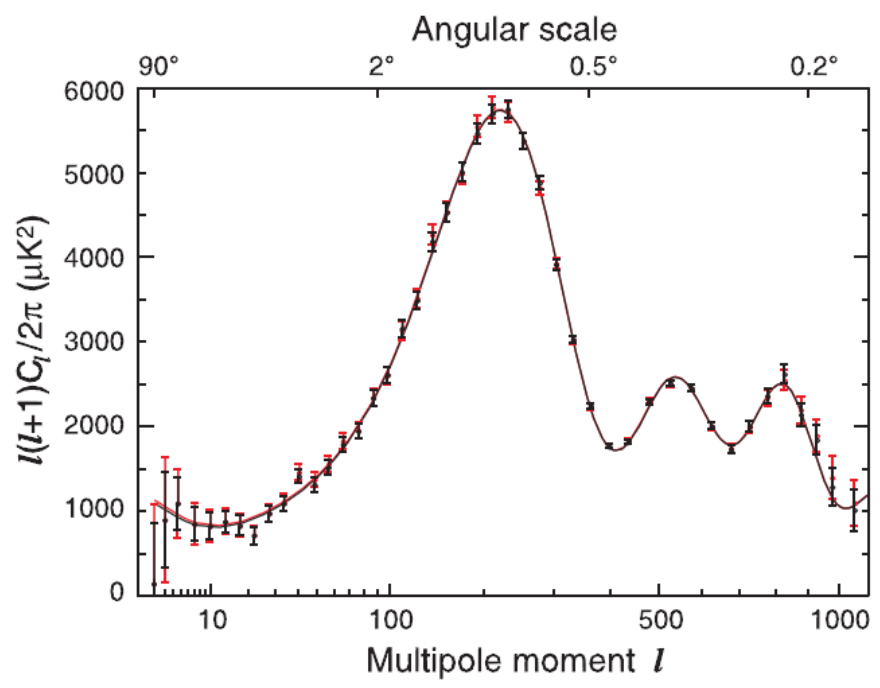

Figura 3.5: Espectro de potências do CMB obtido pelo WMAP9 retirado de Hinshaw et al. $(2013)$.

O primeiro pico do gráfico 3.5 é de especial interesse pois fornece informações sobre a geometria do universo. A posição deste pico está relacionada com a distância máxima que uma onda acústica pode percorrer no plasma primordial, o chamado horizonte acústico (Lazkoz e Majerotto, 2007)

$$
r_{s}=\int_{0}^{t_{r e c}} c_{s} \frac{d t}{a}=\int_{z_{r e c}}^{\infty} c_{s} \frac{d z}{H(z)}
$$

onde $z_{\text {rec }}$ é o redshift de quando ocorre a recombinação. A abertura angular relacionada com (3.34) é dada por:

$$
\theta_{A}=\frac{r_{s}}{d_{A}}
$$

onde $d_{A}$ é a distância de diâmetro angular. Usando (3.33) obtém-se:

$$
l_{A}=\frac{\pi}{\theta_{A}} .
$$

Em geral se usa o chamado shift parameter $(\mathrm{R})$, que é a razão entre os primeiros picos no modelo estudado e em um modelo de referência (neste caso, Einstein-De Sitter): 


$$
R \equiv 2 \frac{l_{A}}{l_{A}^{\prime}} \approx H_{0} \sqrt{\Omega_{m 0}} \int_{0}^{z_{r e c}} \frac{d z}{H(z)}
$$

onde o fator 2 é introduzido para conciliar diferentes definições usadas na literatura. Em (3.37) também é apresentada a aproximação normalmente usada, que pode ser deduzida da seguinte maneira: A equação (3.34) para um modelo composto apenas de matéria e radiação é (Lazkoz e Majerotto, 2007):

$$
r_{s}^{\prime}=\frac{1}{H_{0} \sqrt{\Omega_{m 0}}} \int_{0}^{a_{r e c}} \frac{c_{s} d a}{\left(a+a_{e q}\right)^{1 / 2}}=\frac{2 c_{s}}{H_{0} \sqrt{\Omega_{m 0}}}\left(\left(a_{e q}+a_{r e c}\right)^{1 / 2}+a_{e q}^{1 / 2}\right),
$$

onde se usou $\Omega_{r 0}=\Omega_{m 0} a_{e q}$, sendo $a_{e q}$ o fator de escala em que ocorre a igualdade entra as densidades de matéria e radiação. Para o modelo de referência obtemos

$$
r_{s}^{\prime}=\frac{c_{s}}{H_{0}} a_{r e c}^{1 / 2}
$$

enquanto a distância de diâmetro angular no modelo é dada por:

$$
d_{A}^{\prime}=\frac{2 a_{r e c}}{H_{0}} .
$$

Para um modelo arbitrário sabe-se que em altos redshifts a matéria domina, logo é razoável desprezar os termos de energia escura no cálculo de $r_{s}$. Nesta aproximação podemos escrever

$$
r_{s}=\frac{c_{s}}{H_{0} \sqrt{\Omega_{m 0}}} a_{r e c}^{1 / 2} .
$$

Finalmente, combinando as equações (3.39), (3.40) e (3.41) obtém-se a expressão desejada (3.37). O valor do shift parameter obtido pelo WMAP95 foi $R=1,728 \pm 0,016$ (Bennett et al., 2013). Convêm notar que a expressão (3.37) é valida apenas para o caso plano, a expressão geral é (Davis et al., 2007)

$$
R=\sqrt{\frac{\Omega_{m 0}}{\Omega_{\kappa 0}}} \sinh \left[\Omega_{\kappa 0}^{1 / 2} \int_{0}^{z_{r e c}} \frac{d z}{E(z)}\right]
$$

para calcular $z_{r e c}$ utilizamos a correção sugerida por Hu e Sugivama (1996), apêndice E.

\footnotetext{
${ }^{5}$ WMAP9 significa os resultados de 9 anos de dados do satélite.
} 


\subsubsection{Oscilações Acústicas de Bárions}

As oscilações acusticas de bárions (BAO, na sigla em inglês) surgem pelo mesmo fenômeno do primeiro pico do CMB, ou seja, quando os bárions e os fótons estavam acoplados as ondas acústicas do plasma imprimiram sua influência na distribuição de bárions, então deve existir uma escala de distância preferencial em que haja o aglomeramento da matéria. Da mesma forma que é feito na radiação cósmica de fundo uma análise estatística do espectro de potência da matéria deve revelar esta escala preferencial, a partir disso temse definida uma régua padrão. Isto significa que medir o BAO é equivalente a medir uma distância.

O gráfico 3.6 esquematiza como surge estas oscilações (Eisenstein et al., 2007): As linhas preta, azul, vermelha e verde são matéria escura, matéria bariônica, radiação e neutrinos, respectivamente. Os bárions e a radiação inicialmente estão acoplados, viajando como um pulso (onda acústica), após a recombinação (que ocorre logo após o quadro superior direito) estas componentes se desacoplam, sendo que a radiação e os neutrinos seguem livremente. A partir dai os bárions tendem a seguir os potenciais da matéria escura que haviam se formado anteriormente, a pequena sobredensidade restante da fase acústica é o fenômeno de BAO.

A escala de $\mathrm{BAO}$ é a distância que a onda acústica percorreu até a recombinação. Pode-se utilizar a equação (3.41) para estimar este valor (Nishimichi et al., 2007), para isso, usando $z_{r e c}=1089, \Omega_{m 0}=0,3, a_{e q}=\frac{\Omega_{r 0}}{\Omega_{m 0}}=\frac{10^{-5}}{0,3}, H_{0}=70 \mathrm{~km} / \mathrm{s} / \mathrm{Mpc}$ e $c_{s}=\frac{c}{\sqrt{3(1+R)}}$, onde $R=\frac{3 \rho_{b}}{4 \rho_{\gamma}} \approx \frac{\Omega_{b}}{(1+z)}$ Substituindo os valores obtém-se

$$
r_{s} \approx 148 M p c
$$

As medidas de BAO fornecem uma distância de diâmetro angular, porém, há uma diferença se o efeito for medido perpendicularmente ou paralelamente a linha de visada. Paralelamente temos a distância de diamêtro angular, equação (3.13), no caso perpendicular, a distância é apenas o redshift dividido pelo parâmetro de Hubble

$$
d_{\|}=\frac{z}{H(z)} .
$$

As observações, por serem estatísticas, medem ambas as distâncias, sendo que o que se obtém é uma distância efetiva a qual pode ser escrita como (Eisenstein et al., 2005): 

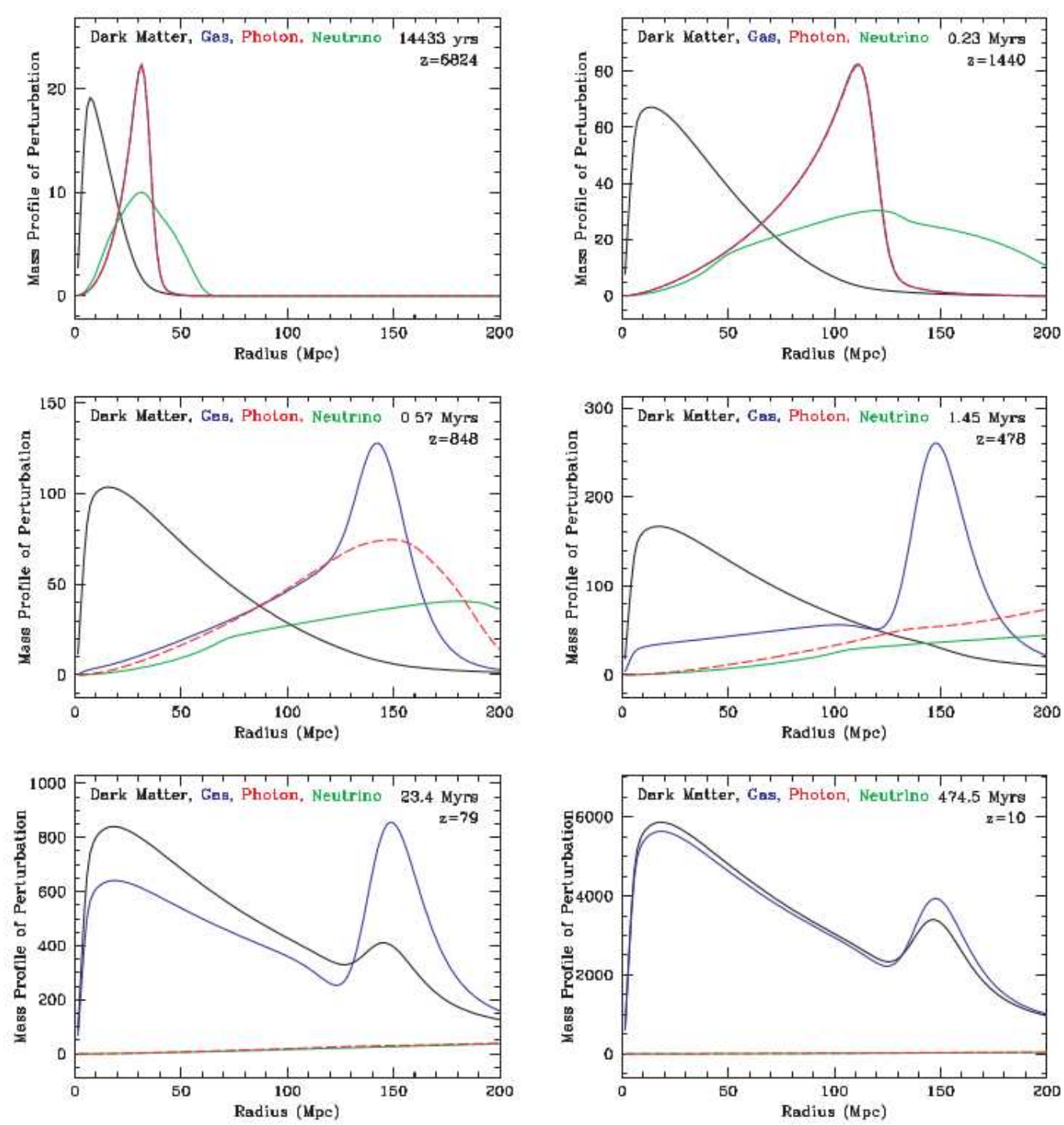

Figura 3.6: Esquematização da oscilação acústica de bárions, retirado de Eisenstein et al. (2007).

$$
d_{V}(z) \equiv\left(\frac{(1+z)^{2} d_{A}^{2}(z) z}{H(z)}\right)^{1 / 3},
$$

o primeiro dado de BAO foi obtido por Eisenstein et al. (2005) usando o SDSS (Sloan Digital Sky Survey) no redshift $z=0,35$. Outros dados surgiram posteriormente, a tabela 3.1 mostra os valores medidos, seus redshifts e as referências. 
Tabela 3.1 - Dados de BAO. A tabela abaixo foi retirada de Hinshaw et al. (2013).

\begin{tabular}{lccc}
\hline \hline redshift & $r_{s} / D_{V}(z)$ & Data Set & Ref. \\
\hline 0,10 & $0,336 \pm 0,015$ & 6dFGRS & Beutler et al. (2011) \\
0,35 & $0,113 \pm 0,002$ & SDSS-DR7-rec & Padmanabhan et al. (2012) \\
0,57 & $0,073 \pm 0,001$ & SDSS-DR9-rec & Anderson et al. (2013) \\
0,44 & $0,0916 \pm 0,0071$ & WiggleZ & Blake et al. (2012) \\
0,60 & $0,0726 \pm 0,0034$ & WiggleZ & Blake et al. (2012) \\
0,73 & $0,0592 \pm 0,0032$ & WiggleZ & Blake et al. (2012) \\
\hline
\end{tabular}

$\mathrm{Na}$ nossa análise estatística utilizamos apenas o dado obtido por Eisenstein através da combinação adimensional

$$
A(z)=d_{V}(z) \frac{\sqrt{\Omega_{m} H_{0}^{2}}}{z} .
$$

Em $z=0,35$ a quantidade $A$ vale $0,469 \pm 0,017$.

\subsubsection{Contornos de Confiança}

Para finalizar mostramos na figura 3.7 os contornos de confiança de $\Omega_{v}$ e $\Omega_{m}$ obtidos usando os dados de SNe, CMB e BAO (Amanullah et al., 2010; Suzuki et al., 2012). Apesar destas observações fornecerem resultados bem distintos, a análise conjunta restringe bastante o espaço de parâmetros, levando ao modelo $\Lambda C D M$, discutido no próximo capítulo. Os dados de BAO são praticamente independentes da densidade de energia do vácuo, enquanto CMB indica um universo aproximadamente plano. As SNe, apesar de serem compatíveis com $\kappa=0$, apontam para um universo curvo, o que leva a chamada tensão SNe-CMB, que discutiremos com mais detalhes no capítulo 6 . 


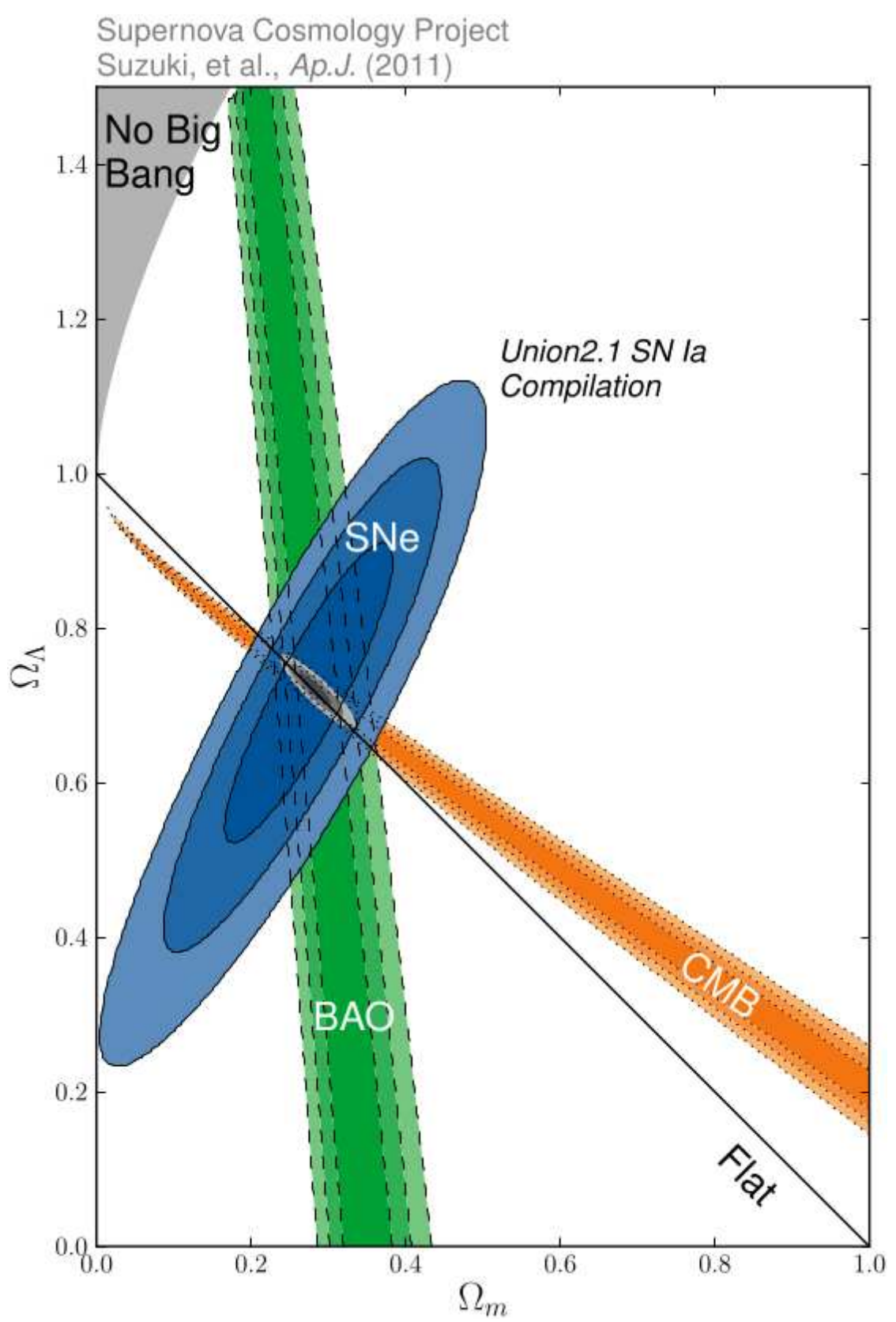

Figura 3.7: Contornos de confiança dos dados de Sne, CMB e BAO, retirado de Suzuki et al. (2012). 
Capítulo 4

\section{O Modelo de Concordância Cósmica e Energia Escura}

No capítulo anterior a cosmologia padrão de FRW foi estudada, até 1998 o modelo padrão era o de Einstein-De Sitter, porém, observações recentes sugerem a presença de uma nova componente, a chamada energia escura. Neste capítulo iremos discutir o modelo $\Lambda C D M$, os seus problemas e a energia escura.

\subsection{O Modelo $\Lambda C D M$}

Conforme discutido anteriormente o universo é dominado por cerca de $1 / 3$ de matéria (escura e bariônica) e 2/3 por energia escura. O candidato mais natural para a energia escura é o vácuo, porém esta não é a única possibilidade, existem diversos outros candidatos que podem fazer o papel de energia escura. Na seção 4.2 iremos discutir os problemas desse modelo, nesta seção trataremos da teoria.

O espectro angular de potências das anisotropias da radiação cósmica de fundo implica que a geometria espacial do universo é plana $\left(\Omega_{\text {total }}=1, \Omega_{\kappa}=0\right)$. Este resultado obtido inicialmente pelo WMAP foi recentemente confirmado com maior precisão pelo experimento do sátelite Planck (Ade et al., 2013). Desta forma, o modelo $\Lambda C D M$ padrão não tem curvatura. Neste caso, a equação de Friedmann pode ser escrita como:

$$
\begin{gathered}
H^{2}=H_{0}^{2}\left(\Omega_{r 0} a^{-4}+\Omega_{m 0} a^{-3}+\Omega_{v 0}\right), \\
\Omega_{m 0}+\Omega_{r 0}+\Omega_{v 0}=1
\end{gathered}
$$

onde $\Omega_{m 0}$ inclui tanto os bárions quanto a matéria escura, geralmente a radiação é desprezada pois ela é dominante apenas em altos redshifts, com as expressões acima simplificadas 
para

$$
H^{2}=H_{0}^{2}\left(\Omega_{m 0} a^{-3}+\Omega_{v 0}\right)
$$

onde $\Omega_{m 0}+\Omega_{v 0}=1$. É possível integrar a equação acima e obter uma expressão para o fator de escala em função do tempo (Lima e Basilakos, 2011):

$$
a(t)=\left(\frac{1-\Omega_{v 0}}{\Omega_{v 0}}\right)^{1 / 3} \sinh ^{2 / 3}\left(\frac{3 H_{0} \sqrt{\Omega_{v 0}}}{2} t\right),
$$

na equação acima já se desprezou o efeito da radiação, desta forma $\Omega_{m 0}=1-\Omega_{v 0}$. O parâmetro de Hubble é obtido derivando o fator de escala:

$$
H(t)=H_{0} \sqrt{\Omega_{v 0}} \operatorname{coth}\left(\frac{3 H_{0} \sqrt{\Omega_{v 0}}}{2} t\right) .
$$

Usando as definições (2.11) obtem-se os parâmetros de densidade do modelo $\Lambda C D M$ :

$$
\Omega_{m}=\frac{\Omega_{m 0}(1+z)^{3}}{\Omega_{m 0}(1+z)^{3}+\Omega_{\Lambda 0}}
$$

e

$$
\Omega_{v}=\frac{\Omega_{v 0}}{\Omega_{m 0}(1+z)^{3}+\Omega_{v 0}}
$$

Outro parâmetro importante neste modelo é a desaceleração, definida por (2.15), utilizando as equações de Friedmann e os parâmetros de densidade:

$$
q=\frac{1}{2}-\frac{3}{2} \Omega_{v}
$$

o modelo $\Lambda C D M$ é acelerado atualmente, porém era desacelerado em altos redshifts, a transição acontece quando $q=0$ e depende dos parâmetros cosmológicos:

$$
z_{T}=\left(\frac{2 \Omega_{v 0}}{1-\Omega_{v 0}}\right)^{1 / 3}-1,
$$

é interessante analisar o redshift de transição pois ele se modifica para modelos diferentes, então se for possível determina-lo observacionalmente pode-se eliminar alguns destes modelos. O futuro de um universo $\Lambda C D M$ será o modelo de De-Sitter discutido na seção 2.4.2, ou seja, a constante cosmológica dominará completamente e a expansão será exponencial, o gráfico 4.1 mostra a evolução do parâmetro de desaceleração até o futuro distante $(z=-1)$ para diferentes valores de $\Omega_{v 0}$. 


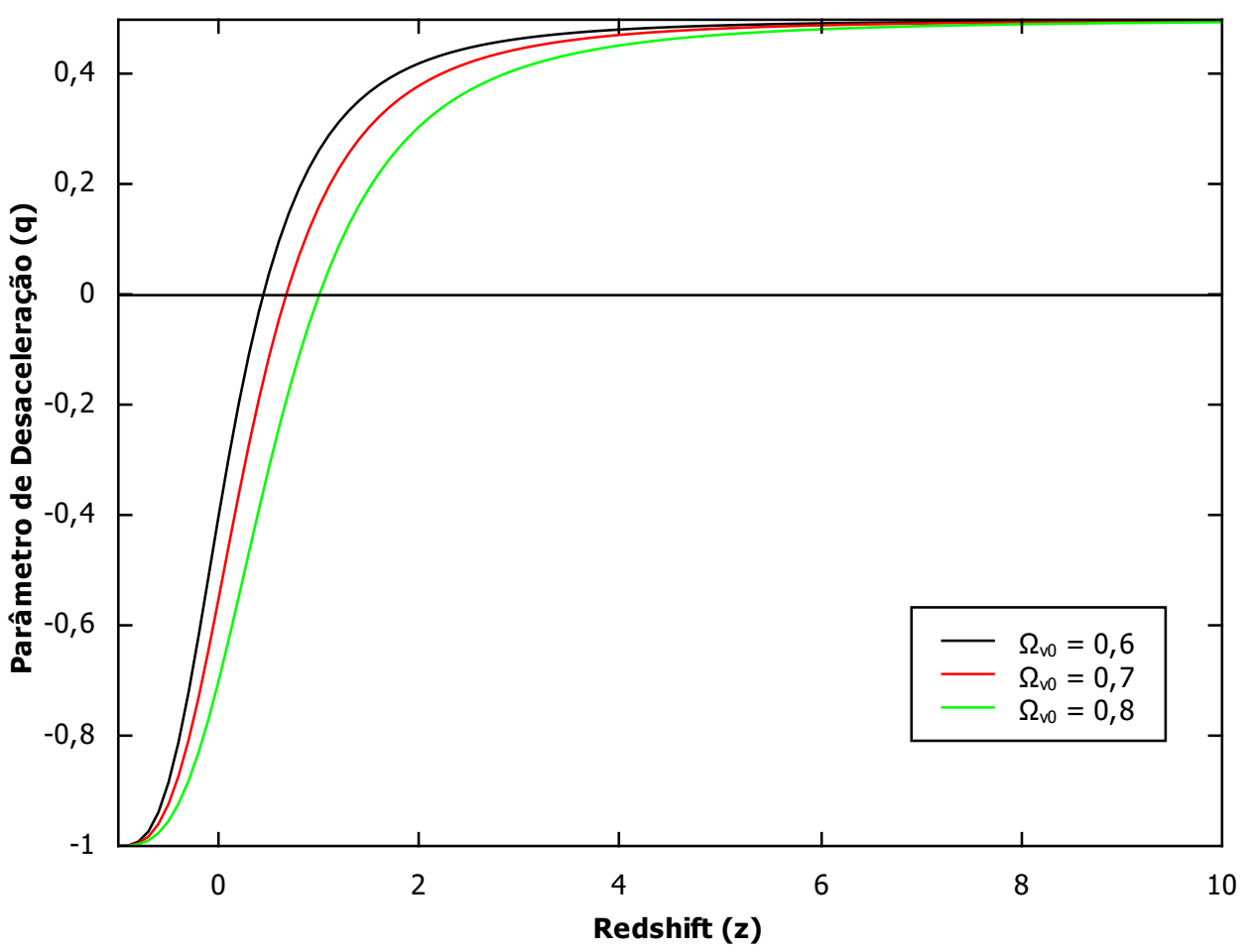

Figura 4.1: Parâmetro de desaceleração em função do redshift para $\Lambda C D M$.

A idade é dada pela equação (2.25), substituindo (4.3):

$$
H_{0} t_{0}=\int_{0}^{1} \frac{d x}{x \sqrt{\Omega_{m 0} x^{-3}+\Omega_{v 0}}}
$$

a integral pode ser feita analiticamente:

$$
H_{0} t_{0}=\frac{2}{3 \sqrt{\Omega_{v 0}}} \ln \left[\left(\frac{1}{\Omega_{m 0}}\right)^{1 / 2}+\left(\frac{\Omega_{v 0}}{\Omega_{m 0}}\right)^{1 / 2}\right],
$$

a figura 4.2 mostra o gráfico da idade, obtida pela equação acima, em função de $\Omega_{v 0}$, para comparação a idade no modelo de Einstein-De Sitter é $H_{0} t_{0}=2 / 3=0,66$ (equação (2.53)), que corresponde à $\Omega_{v 0}=0$ na equação (4.11).

O modelo $\Lambda C D M$ também é conhecido como modelo de concordância cósmica pois ele é o que melhor se ajusta as observações, a figura 3.7 mostra os contornos de confiança obtidos independentemente, a convergência destas observações determinam os seus parâmetros. 


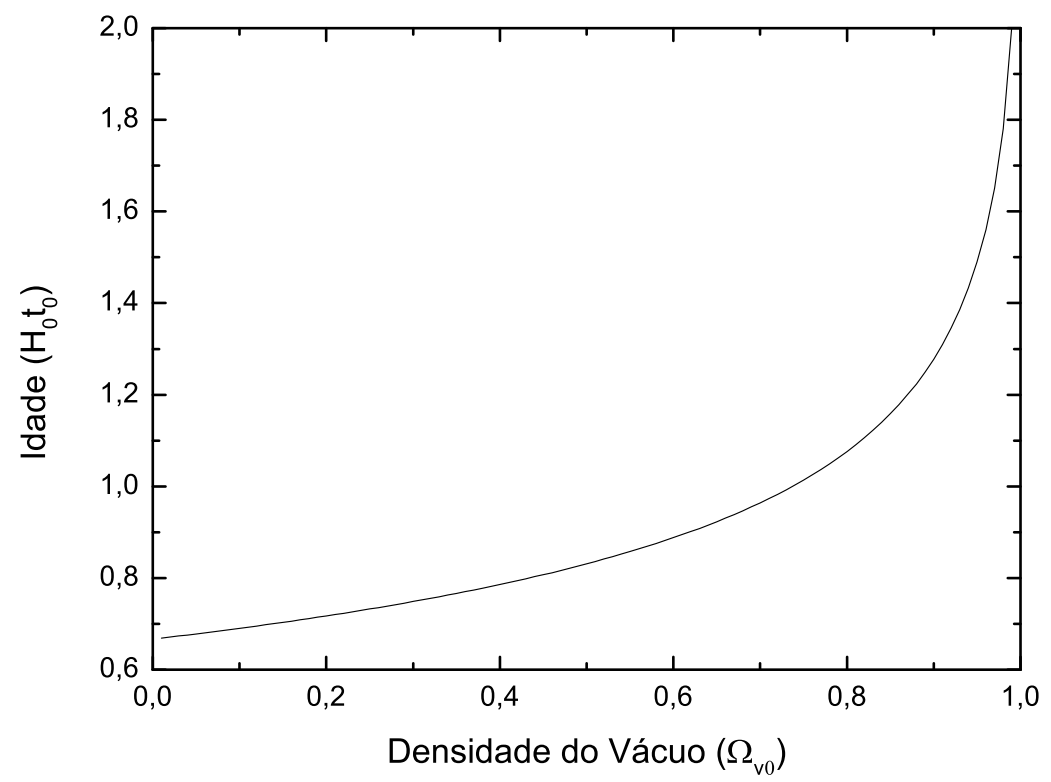

Figura 4.2: Idade do modelo $\Lambda C D M$ em função da densidade de energia do vácuo.

\subsection{Problemas da Constante Cosmológica}

A constante cosmológica sofre de dois problemas principais, nesta seção iremos discutir esses problemas.

\subsubsection{O Problema Original}

O antigo problema da constante cosmológica vem da comparação do valor da densidade de energia do vácuo obtido observacionalmente com o teórico. O artigo de revisão de Weinberg (1989) expõe esse problema e discute algumas soluções, aqui vamos discutir o problema.

No modelo $\Lambda C D M$ cerca de $70 \%$ da densidade de energia do universo esta na forma de vácuo, ou seja:

$$
\rho_{v 0} \approx 0,7 \times \rho_{\text {crit } 0}=0,7 \times \frac{3 H_{0}^{2}}{8 \pi G},
$$

em unidades do sistema internacional a densidade crítica vale $\rho_{\text {crit } 0}=1,88 \times 10^{-26} \mathrm{~h}^{2} \mathrm{~kg} / \mathrm{cm}^{3}$, onde $h$ é a constante de Hubble em unidades de $100 \mathrm{~km} / \mathrm{s} / \mathrm{Mpc}$. Substituindo acima e usando $h=0,72$ : 


$$
\rho_{v 0} \approx 1,32 \times 10^{-26} h^{2} \mathrm{~kg} / \mathrm{m}^{3} \approx 10^{-47} \mathrm{GeV}^{4}
$$

este é o valor observado da densidade de energia da constante cosmológica. Por outro lado, pode-se estimar o seu valor teórico calculando a energia de ponto zero de algum campo quântico com massa $m$ até um corte em $\Lambda \gg m$ (Weinberg, 1989):

$$
\rho_{v 0} \approx \frac{\Lambda^{4}}{16 \pi^{2}}
$$

Porém, se a relatividade geral for válida até a escala de Planck podemos tomar $\Lambda \approx$ $(8 \pi G)^{-1 / 2}$, o que leva:

$$
\rho_{v 0} \approx 2 \times 10^{71} \mathrm{GeV}^{4}
$$

Assim, comparando (4.13) com (4.15) vemos que existe uma divergência de 118 ordens de grandeza. Apesar de termos identificado a constante cosmológica com a energia do vácuo pode-se considerar as duas distintamente, sendo que o observado é a sua soma:

$$
\rho_{v 0}=\langle\rho\rangle+\frac{\lambda}{8 \pi G}
$$

onde $\langle\rho\rangle$ é a energia de ponto zero de todos os campos quânticos e $\lambda$ é a constante cosmológica geométrica. Com essa distinção pode-se dizer que os dois termos devem se cancelar quase perfeitamente, para resultar na densidade de energia do vácuo observada. $\mathrm{O}$ problema desse ponto de vista seria saber como $\lambda$ foi adicionada no ínicio da evolução do universo para exatamente cancelar, após 13 bilhões de anos, a energia de ponto zero de todos os campos quânticos existentes no universo (Weinberg, 1989).

Existem algumas tentativas para solucionar este problema, o próprio Weinberg (1989) discutiu algumas possibilidades. Uma extensa discussão do problema pode ser vista no artigo de Nobbenhuis (2006), e mais recentemente em Martin (2012).

\subsubsection{Novo Problema?}

Existe uma nova versão do problema da constante cosmológica também conhecido como problema da coincidência. Como visto anteriormente a densidade de energia do vácuo é constante e a da matéria varia com $a^{-3}$, então espera-se que estas quantidades tenham valores várias ordens de magnitude diferentes ao longo da histórica cósmica, porém, o que 
se mede é que hoje os valores são semelhantes. A questão é: estamos em alguma época especial da história ou existe algum outro motivo para esta semelhança?

É claro que o primeiro motivo é perfeitamente razoável, porém não podemos nos limitar somente a esta explicação sem considerar outras possibilidades, se houver algum motivo físico para tal concidência o problema estará resolvido.

\subsection{Energia Escura}

No modelo de concordância cósmica a fase acelerada é explicada pela presença da constante cosmológica, que esta associada ao vácuo (seção 2.3.4), porém, conforme visto na seção anterior, existem problemas associados à $\Lambda$. Por outro lado, apesar dos dados em geral favorecerem o modelo $\Lambda C D M$, ainda existe a possibilidade de que a causa da aceleração seja outra, por esses motivos é interessante buscar alternativas à constante cosmológica. O termo usado para a componente que causa a aceleração é energia escura, em analogia à matéria escura.

Desde a descoberta da aceleração cósmica surgiram inúmeros candidatos a energia escura, alguns modelos tentam resolver os problemas apresentados anteriormente, outros buscam explicar a aceleração sem introduzir uma nova componente (Lima, 2004). Descreveremos a seguir as principais alternativas para a energia escura, podendo classificá-las em duas categorias: as que preservam a relatividade geral e as que modificam, primeiramente vamos comentar os modelos que modificam, após isso discutiremos os que mantém.

\subsubsection{Modificações da Relatividade}

As teorias mais conhecidas que modificam a relatividade para explicar a aceleração são as teorias $f(R)$ (Sotiriou e Faraoni, 2010). A relatividade geral pode ser obtida pela ação de Einstein-Hilbert, dada por:

$$
S_{E H}=\frac{1}{16 \pi G} \int d^{4} x \sqrt{-g} R,
$$

onde $g$ é o determinante da métrica e $R$ é o escalar de Ricci. Nas teorias $f(R)$ o escalar de Ricci é substituído por uma função genérica:

$$
S=\frac{1}{16 \pi G} \int d^{4} x \sqrt{-g} f(R)
$$


cada função diferente determina uma teoria de gravitação diferente. Com essa liberdade de escolha na função $f$ é possível obter teorias em que não há necessidade de energia escura.

Outra teoria bastante citada é a chamada DGP (Dvali et al., 2000) (nomeada pelas iniciais de seus idealizadores: Dvali, Gabadadze e Porrati). Nesta teoria a gravidade se propaga em $4+1$ dimensões (4 espaciais e 1 temporal), porém, nas dimensões usuais enxergamos a teoria newtoniana em pequenas escalas, em grandes escalas a gravidade é modificada. Neste modelo também não há necessidade de energia escura.

\subsubsection{Modelos XCDM}

Esta classe de modelos é simplismente uma generalização da equação de estado do vácuo, ou seja, há uma componente com pressão negativa cuja equação de estado é dada por:

$$
p_{x}=\omega \rho_{x}
$$

onde $\omega$ deve ser menor que $-1 / 3$ para haver aceleração, sendo o caso padrão $(\Lambda C D M)$ obtido quando $\omega=-1$. Outra generalização feita neste tipo de modelo é permitir que $\omega$ varie com o redshift, normalmente segundo a parametrização (Huterer e Turner, 2001):

$$
\omega(a)=\omega_{0}+\omega_{1} z
$$

ou ainda a sugerida por Chevallier e Polarski (2001) e Linder (2004)

$$
\omega(a)=\omega_{0}+\frac{\omega_{1} z}{1+z}
$$

A primeira parametrização é útil para baixos redshifts, enquanto a segunda funciona também para altos (sempre finita). Como não se conhece a natureza da energia escura é importante determinar se $\omega$ é realmente constante ou se há alguma variação.

O modelo $X C D M$ é bastante usado em testes cosmológicos para verificar se existe algum desvio da constante cosmológica, tanto no valor da equação de estado quanto na sua variação temporal. 


\subsubsection{Gás de Chaplygin}

O gás de Chaplygin 1 é uma componente exótica que tem equação de estado na forma (Bento et al., 2002):

$$
p=-\frac{A}{\rho^{\alpha}}
$$

onde as constantes $A$ e $\alpha$ são positivas, sendo $\alpha=1$ no modelo original enquanto o chamado gás de Chaplygin generalizado tem $0<\alpha<1$. Por possuir pressão negativa esta componente pode desempenhar o papel de energia escura.

O artigo Kamenshchik et al. (2001) sugere uma aplicação do modelo original (com $\alpha=1$ ) para a cosmologia. $\mathrm{O}$ aspecto mais interessante das cosmologias dirigidas pelo gás de Chaplygin (tanto na versão original quanto generalizada) é que sua equação de estado pode interpolar naturalmente entre a matéria não relativística e um regime de energia escura. Consequências deste cenário utilizando os dados de Supernovas tipo Ia foram examinadas por diversos autores. Tais resultados indicam que uma cosmologia dominada completamente pelo gás de Chaplygin é favorecida em comparação com os modelos do tipo $\Lambda C D M$. Bento et al. (2002) mostraram que a localizacão dos picos da CMB fornecem vínculos nos parâmetros livres do modelo, enquanto Dev et al. (2003) investigaram os vínculo na equação de estado do gás de Chaplygin utilizando estatística de lentes gravitacionais fortes em estimativas de idade em altos-z. Mais recentemente, diversas propriedades do chamado gás de Chaplyging simplificado foi discutido por Sandvik et al. (2004), Lima et al. (2008) e Lima et al. (2009).

\subsubsection{Criação de Matéria Escura}

Neste modelo a pressão negativa responsável pela aceleração vem da criação de partículas de matéria escura, logo não há necessidade da energia escura. A criação de matéria é gravitacionalmente induzida. Schrödinger (1939) foi quem primeiro mostrou que esse fenômeno pode ser microscopicamente justificado. 1992):

\footnotetext{
${ }^{1}$ Nomeado em homenagem ao físico soviético Sergey Chaplygin, que o introduziu em 1904 num problema de aerodinâmica (Chaplvgin, 1904).
} 


$$
p_{c}=-\frac{\rho_{m} \Gamma}{3 H}
$$

onde $\Gamma$ é a taxa de criação. Ao se determinar esta taxa é possível obter expressões para os parâmetros cosmológicos e determinar toda a história cósmica. Existem vários artigos que exploram este fenômeno na cosmologia, como exemplo citamos o modelo de Lima et al. (2010) em que a taxa é dada por:

$$
\Gamma=3 \alpha \frac{\rho_{\text {crit } 0}}{\rho_{m}},
$$

onde $\alpha$ é um parâmetro do modelo. A dinâmica deste modelo é idêntica a $\Lambda C D M$ com a identificação $\alpha=\Omega_{\Lambda 0}$.

\subsubsection{Quintessência}

Quintessência é uma forma de energia escura dinâmica normalmente implementada como um campo escalar. Veremos no próximo capítulo que é interessante considerar a energia do vácuo dependente do tempo, lá faremos uma exposição mais detalhada das leis de decaimento fenomenológicas.

Considerando um campo escalar $\phi$ que só dependa do tempo e um potencial $V(\phi)$ deste campo. É possível mostrar (Weinberg, 2008) que existe uma pressão e uma densidade associada a este campo:

$$
\rho_{\phi}=\frac{1}{2} \dot{\phi}^{2}+V(\phi)
$$

$\mathrm{e}$

$$
p_{\phi}=\frac{1}{2} \dot{\phi}^{2}-V(\phi)
$$

em analogia com (2.7) define-se

$$
\omega_{\phi}=\frac{p_{\phi}}{\rho_{\phi}}
$$

nestes modelos nem sempre $\omega_{\phi}$ é constante ou igual a -1 , como no caso da constante cosmológica. Substituindo (4.25) e (4.26) em (2.6) obtém-se a equação de conservação para o campo escalar: 


$$
\ddot{\phi}+3 H \dot{\phi}+V^{\prime}(\phi)=0
$$

onde linha é derivada com relação a $\phi$. A expressão (4.28) é análoga a equação de uma partícula com massa unitária que se move unidimensionalmente na coordenada $\phi$ sujeita a um potencial $V(\phi)$ e uma força de fricção - $3 H \dot{\phi}$, desta forma o campo se move em direção a valores menores do potencial, chegando ao repouso em algum minímo local. Neste repouso a derivada do campo se anula e (4.27) vale -1, efetivamente igual a constante cosmológica.

Para prosseguir normalmente se postula um potencial atrativo e se estuda as suas consequências cosmológicas.

Existem outros modelos interessantes descritos como cosmologias com $\Lambda=\Lambda(t)$, ou seja, onde a densidade de energia do vácuo é uma função do tempo. Tais modelos involvem uma interação no setor escuro e serão discutidas a seguir. 
Capítulo 5

\section{Modelos Com Interação no Setor Escuro}

Neste capítulo vamos tratar de modelos com interação no setor escuro considerando que a componente de energia escura é representada por uma constante cosmológica que varia com o tempo, $\Lambda=\Lambda(t)$. Ao se tratar a constante cosmológica como uma componente material não existe nenhuma razão para considerá-la constante, contanto que a lei de conservação da energia total (2.6) permaneça válida. No contexto de teoria de campos qualquer componente (dinâmica ou não) que contribua na densidade de energia do vácuo se comporta como uma constante cosmológica.

Normalmente, se a densidade de energia do vácuo varia com o tempo, sua energia deve estar sendo transferida para algum lugar. Neste trabalho vamos assumir que há uma transferência para a matéria escura. O principal motivo disso é que se desconhece a natureza de ambas componentes, então pode-se assumir uma interação entre elas, porém existem outras possibilidades, por exemplo decaimento em radiação (Lima, 1996; Lima et al., 2000). Apesar de, em geral, o sentido da transeferência de energia ser para matéria escura, as equações são simétricas, podendo haver inversão do sentido da interação.

Em geral, os exemplos de componentes que decaem vindos da teoria de campos não fornecem uma expressão simples para $\Lambda$, por outro lado, pode-se postular uma lei de decaimento a fim de explorar suas consequências cosmológicas, apesar dessa abordagem ser imcompleta ainda assim é possível obter informações sobre a viabilidade da interação.

Uma das motivações principais para a interação no setor escura é resolver o problema da coincidência. Na seção 5.2 discutiremos como a interação alivia este problema.

No final deste capítulo (seção [5.3) alguns exemplos de leis de decaimento serão apresentadas, porém num primeiro momento vamos discutir a teoria geral. 


\subsection{Teoria}

Vamos descrever o universo como uma mistura de $N$ fluidos perfeitos com quadrivelocidade $u_{\mu}^{N}$. O tensor energia momento total é dado por

$$
T_{\mu \nu}=\sum_{N} T_{\mu \nu}^{N}=\sum_{N}\left[-p_{N} g_{\mu \nu}+\left(\rho_{N}+p_{N}\right) u_{\mu}^{N} u_{\nu}^{N}\right]
$$

As componentes de $T_{\mu}^{\nu}$ são dadas por:

$$
T_{0}^{0}=\sum_{N} \rho_{N} \equiv \rho_{T}, \quad T_{j}^{i}=-\sum_{N} p_{N} \delta_{j}^{i} \equiv-p_{T} \delta_{j}^{i},
$$

onde $\rho_{T}$ e $p_{T}$ são a densidade de energia e a pressão total no sistema de coordenada comóvel $\left(u_{N}^{0}, u_{N}^{i}\right)=(1,0)$, respectivamente. A lei de conservação covariante local para a mistura é dada por

$$
\nabla_{\nu} T^{\mu \nu}=0
$$

a expressão acima pode ser calculada utilizando (5.1). Contraindo a expressão acima com a quadrivelocidade $\left(u_{\nu}^{N} \nabla_{\mu} u_{N}^{\nu}=0\right)$, obtemos o seguinte resultado

$$
\sum_{N}\left[u_{N}^{\mu} \nabla_{\mu} \rho_{N}+\left(p_{N}+\rho_{N}\right) \nabla_{\mu} u_{N}^{\mu}\right]=0
$$

esta é a equação de conservação escrita em uma forma mais explícita. Para a métrica de FRW podemos simplificá-la, adotando o sistema de coordenada comóvel $\left(u_{N}^{\mu}=\delta_{0}^{\mu}\right)$, para o qual obtemos:

$$
\nabla_{\mu} u_{N}^{\mu}=3 H
$$

com a relação (5.4) se reduzindo para

$$
\sum_{N}\left[\dot{\rho}_{N}+3 \frac{\dot{a}}{a}\left(p_{N}+\rho_{N}\right)\right]=0,
$$

onde a soma se refere a todas as componentes interagentes, ou seja, a conservação de energia ocorre apenas para o fluido composto. A equação acima é a forma mais geral para a lei de conservação, válida para mistura de várias componentes, podendo existir interação entre duas ou mais componentes. 
Até esse ponto não especificamos a natureza das componentes envolvidas, agora assumiremos que há interação apenas no setor escuro (entre energia escura e matéria escura). Suporemos também que a energia escura é representada pela densidade de energia do $\operatorname{vácuo}\left(p_{v}=-\rho_{v}\right)$. Neste caso temos $\dot{\rho}_{m}+3 H \rho_{m}=-\dot{\rho}_{v}$. Sem perda de generalidade, podemos escrever:

$$
\begin{gathered}
\dot{\rho}_{v}=-Q, \\
\dot{\rho}_{m}+3 H \rho_{m}=Q,
\end{gathered}
$$

onde $Q$ é a taxa de transferência de energia entre matéria escura e vácuo. Se a energia for transferida do vácuo para a matéria escura $Q$ é positivo, caso contrário é negativo, para $Q=0$ o caso padrão onde não há interação ( $\rho_{v}$ constante) é restaurado. Analisando as equações (5.7) e (5.8) percebe-se que as densidades de energia não seguem as suas expressões usuais (a do vácuo constante e a da matéria escura proporcional a $a^{-3}$ ).

A taxa de transferência de energia é uma quantidade fundamental pois ela determina como ocorre a interação e qual componente cede e qual recebe energia. Em princípio $Q$ poderia ser obtida conhecendo a natureza física das componente, porém como ainda não existe um concenso sobre isso pode-se postular qual é a forma de interação a fim de estudar as suas consequências. Uma vez determinada como ocorre a interação pode-se integrar as equações (5.7) e (5.8) e obter o parâmetro de Hubble através da primeira equação de Friedmann 1 :

$$
H^{2}=\frac{8 \pi G}{3} \sum_{i} \rho_{i}
$$

Com isso é possível fazer testes cosmológicos e verificar se a forma de interação estudada é viável.

É preciso deixar clara a diferença entre matérica bárionica e escura neste contexto pois a primeira não esta interagindo, ou seja:

$$
\dot{\rho}_{b}+3 H \rho_{b}=0,
$$

\footnotetext{
${ }^{1}$ Por simplicidade, nesta seção consideraremos $\Omega_{\kappa}=0$.
} 
isto significa que a densidade dos bárions não se modifica $\left(\rho_{b}=\rho_{b 0} a^{-3}\right)$ pois a interação ocorre somente no setor escuro.

Dependendo da forma da interação pode ser difícil integrar as equações de conservação, sendo necessário recorrer a técnicas numéricas. É conveniente estudar o sistema em variáveis adimensionais, introduzindo outra equação ao sistema:

$$
\dot{H}=4 \pi G \rho_{v}-\frac{3}{2} H^{2},
$$

esta equação vem da combinação das duas equações de Friedmann (2.4) e (2.5). Trocando para variáveis adimensionais:

$$
\begin{gathered}
\Omega_{v}=\frac{8 \pi G}{3 H^{2}} \rho_{v}, \\
\Omega_{m}=\frac{8 \pi G}{3 H^{2}} \rho_{m},
\end{gathered}
$$

$\mathrm{e}$

$$
E=\frac{H}{H_{0}}
$$

Mudando a variável $t$ por $\ln a$ as equações (5.7) e (5.8) e (5.11) se transformam:

$$
\begin{gathered}
\Omega_{v}^{\prime}=3 \Omega_{v}\left(1-\Omega_{v}\right)-\widehat{Q}, \\
\Omega_{m}^{\prime}=-3 \Omega_{v} \Omega_{m}+\widehat{Q},
\end{gathered}
$$

$\mathrm{e}$

$$
E^{\prime}=\frac{3}{2} E\left(\Omega_{v}-1\right)
$$

onde linha é derivada com relação a lna e se definiu uma nova função relacionada com a taxa de transferência de energia

$$
\widehat{Q}=\frac{8 \pi G}{3 E^{3} H_{0}^{3}} Q
$$

de maneira geral $\widehat{Q}$ pode ser função de todos os parâmetros envolvidos e do tempo. A densidade de energia dos bárions é determinada pela equação de Friedmann (5.9): 


$$
\Omega_{b}=\frac{8 \pi G}{3 H^{2}} \rho_{b}=1-\Omega_{v}-\Omega_{m}
$$

o parâmetro de desaceleração é dado por:

$$
q=\frac{1}{2}-\frac{3}{2} \Omega_{v}
$$

ou seja, é a mesma forma do modelo padrão (4.8) pois a forma das equações de Friedmann não se modificam, a diferença será em $\Omega_{v}$. Na seção 5.3 trataremos de interações específicas, porém antes disso vamos ver como a interação ajuda a aliviar o problema da coincidência.

\subsection{Problema da Coincidência}

Na seção 4.2 .2 discutimos qual a origem do problema da coincidência, vejamos agora como a interação ajuda a aliviar este problema.

Para visualizar este problema de maneira analítica é útil analisar a razão entre as densidade de energia da matéria e energia escura (Zimdahl, 2012):

$$
r=\frac{\rho_{m}}{\rho_{v}}=r_{0} a^{-\xi}
$$

onde $\xi$ é um parâmetro de escala. Para $\Lambda C D M$ o valor de $\xi$ é 3, ou seja, espera-se que $r$ varie muito ao longo da história cósmica. Valores de $\xi$ menores aliviam o problema da coincidência. O caso extremo seria $\xi=0$, ou seja, a razão é estacionária.

No contexto de interação $r$ evolue da seguinte forma:

$$
\frac{r^{\prime}}{r}=-3+\frac{\Omega_{v}+\Omega_{m}}{\Omega_{v} \Omega_{m}} \widehat{Q}
$$

onde se utilizamos as equações (5.15) e (5.16). Se $r$ apresentar um valor assintótico o problema da coincidência estaria explicado, pois conforme o tempo evolui a razão se torna constante. Neste caso, devemos ter $r^{\prime}=0$, o que ocorre para

$$
\widehat{Q}=3 \frac{\Omega_{v} \Omega_{m}}{\Omega_{v}+\Omega_{m}}
$$

para $a \rightarrow \infty$, ou seja, conforme o tempo aumenta a razão vai se tornando constante. 


\subsection{Formas de Interação}

Existem inúmeras propostas fenomenológicas para descrever a interação no setor escuro, nesta seção discutiremos algumas delas.

Nesta seção trataremos de algumas abordagens para interação, os modelos foram escolhidos para representar a grande variedade existente, não sendo exaustiva, a tabela [5.1, retirada de Overduin e Cooperstock (1998), apresenta uma lista de várias leis fenomenológicas de decaimento, onde há também uma revisão sobre o assunto.

\subsubsection{Modelo $Q=A_{v} \rho_{v}+A_{m} \rho_{m}$}

Primeiramente vamos tratar do modelo cuja taxa de transferência de energia é proporcional as densidades de energia do setor escura:

$$
Q=A_{v} \rho_{v}+A_{m} \rho_{m}
$$

Os coeficientes $A_{v}$ e $A_{m}$ não necessariamente são constantes. Este modelo é bem geral e tem como caso particular vários outros modelos, basta escolher os coeficientes, por exemplo, em Caldera-Cabral et al. (2009) há uma análise dinâmica das equações cosmológicas para dois modelos dessa forma:

$$
A_{i}=3 \alpha_{i} H
$$

e

$$
A_{i}=3 \Gamma_{i},
$$

onde $i$ pode ser vácuo ou matéria escura, $\alpha_{i}$ e $\Gamma_{i}$ são constantes. O primeiro destes modelos tem a taxa de transferência proporcional ao parâmetro de Hubble, cuja motivação é puramente simplicidade matemática, o segundo modelo possui taxa proporcional as densidades de energia e sua motivação surge de outros modelos similares. Com estas leis fenomonológicas os autores analisam o comportamento dinâmico das equações de Friedmann. 
Tabela 5.1 - Leis de Decaimento, tabela retirada de Overduin e Cooperstock (1998).

\begin{tabular}{|c|c|c|c|}
\hline Lei de Decaimento & Referências & Lei de Decaimento & Referências \\
\hline$\Lambda \propto t^{-2}$ & 2 & $\Lambda \propto \beta a^{-2}+H^{2}$ & 3 \\
\hline$\Lambda \propto T^{4}$ & 4 & $\Lambda \propto t^{-2}+\beta t^{-2 / l}$ & 5 \\
\hline$\Lambda \propto T^{\beta}$ & 6 & $\Lambda \propto C+e^{-\beta t}$ & 7 \\
\hline$\Lambda \propto e^{-\beta a}$ & 8 & $\Lambda \propto C+\beta a^{-2}+H^{2}$ & 9 \\
\hline$\frac{d \Lambda}{d t} \propto \Lambda^{\beta}$ & 10 & $\Lambda \propto \beta a^{-m}+H^{2}$ & 11 \\
\hline$\Lambda \propto a^{-2}$ & 12 & $\Lambda \propto H^{2}$ & 13 \\
\hline$\Lambda \propto a^{-4(1+\epsilon)}$ & 14 & $\Lambda \propto(1+\beta H)\left(H^{2}+\kappa / a^{2}\right)$ & 15 \\
\hline$\Lambda \propto a^{-m}$ & 16 & $\Lambda \propto t^{-1}(\beta+t)^{-1}$ & 17 \\
\hline$\frac{d \Lambda}{d t} \propto a H^{n} \Lambda$ & 18 & $\frac{d \Lambda}{d t} \propto \beta \Lambda-\Lambda^{2}$ & 19 \\
\hline$\frac{d \Lambda}{d t} \propto H^{3}$ & 20 & $\Lambda \propto a^{-3}$ & 21 \\
\hline$\Lambda \propto C+\beta a^{-m}$ & 22 & $\Lambda \propto a^{-2}+\beta a^{-4}$ & 23 \\
\hline$\Lambda \propto t^{l-2}+\beta t^{2(l-1)}$ & 24 & $\Lambda \propto H^{2}+\beta a H \frac{d H}{d a}$ & 25 \\
\hline
\end{tabular}

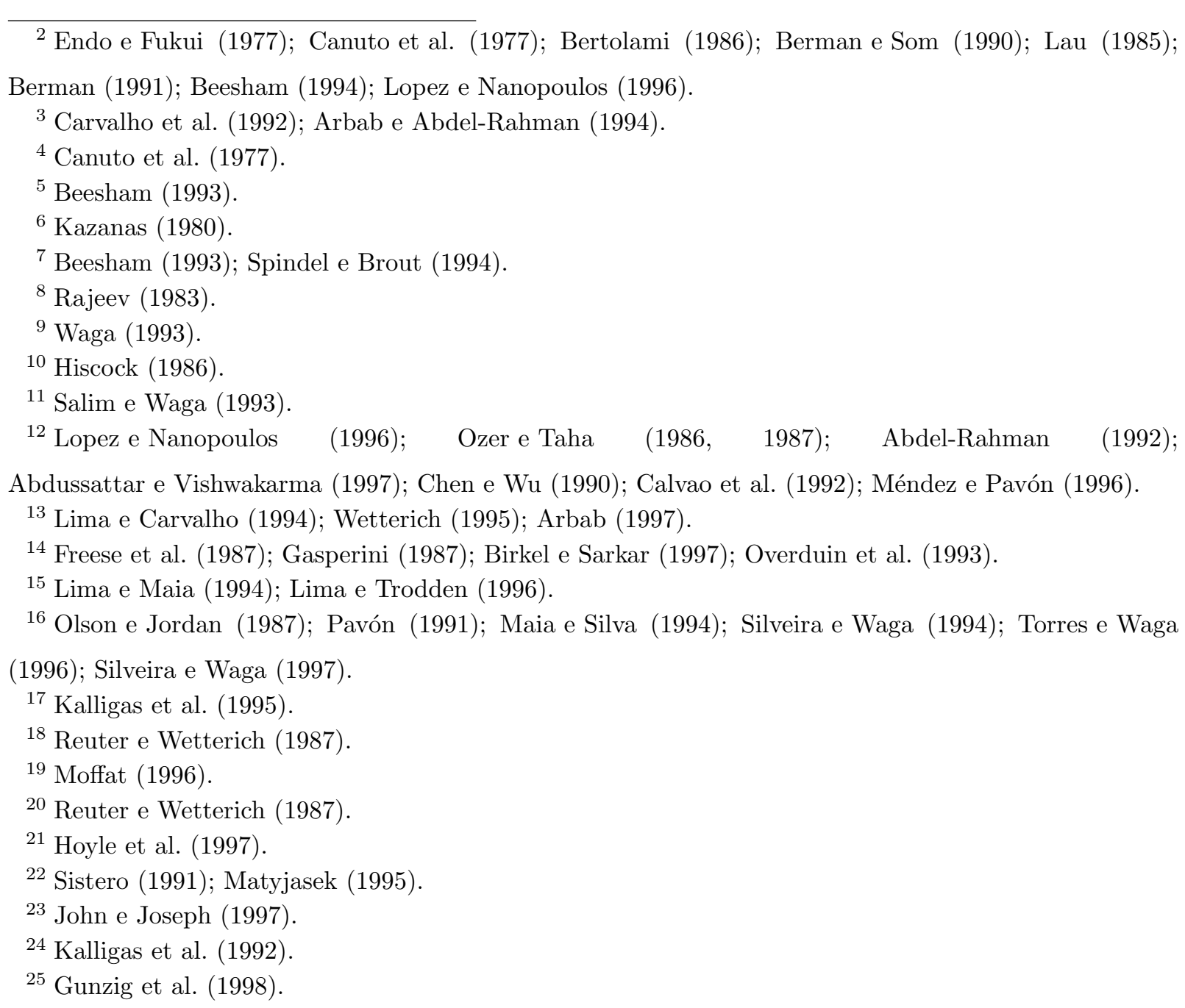




\subsubsection{Modelo de Wang e Meng}

Wang e Meng (2005) propuseram uma abordagem alternativa para a interação, ao invés de especificar uma lei para $\Lambda$ eles propuseram quantificar os efeitos da interação na densidade da matéria:

$$
\rho_{m}=\rho_{m 0} a^{-3+\epsilon}
$$

o parâmetro $\epsilon$ surge devido a interação, ou seja, há um desvio do comportamento padrão da densidade da matéria escura. Se $\epsilon$ for positivo a matéria decai mais lentamente, ou seja, há transferência de energia do vácuo para a matéria escura, caso o contrário $\epsilon$ sera negativo. Espera-se também que $|\epsilon| \ll 1$ pois as observações limitam a força da interação. A partir das equaões (5.7) e (5.8) pode-se achar a densidade de energia do vácuo

$$
\rho_{v}=\widetilde{\rho}_{v 0}+\frac{\epsilon \rho_{m 0}}{3-\epsilon} a^{-3+\epsilon}
$$

onde $\widetilde{\rho}_{v 0}$ representa o estado de mais baixa energia do vácuo, o valor da densidade de energia hoje é $\rho_{v 0}=\widetilde{\rho}_{v 0}+\frac{\epsilon \rho_{m 0}}{3-\epsilon}$. A partir das equações (5.27) e (5.28) é possível obter o parâmetro de Hubble e fazer testes cosmológicos. Alcaniz e Lima (2005) e Jesus et al. (2008) expandiram o trabalho de Wang e Meng incluindo bárions e para um modelo XCDM interagente, respectivamente.

\subsubsection{Modificações na Densidade da matéria}

Uma abordagem alternativa ao modelo de Wang e Meng é modificar a densidade da matéria com uma função multiplicativa (Zimdahl, 2008, 2012):

$$
\rho_{m}=\rho_{m 0} a^{-3} f(a)
$$

onde $f(a)$ é a modificação devido a interação. A equação de conservação para a energia escura pode ser reescrita da seguinte forma:

$$
\dot{\rho}_{x}=-3 H\left(1+\omega_{e f}\right) \rho_{x}
$$

onde se esta usando $p_{x}=\omega \rho_{x}$ e o parâmetro $w_{e f}$ é dado por 


$$
\omega_{e f}=\omega+\frac{\dot{f}}{3 H f} \frac{\rho_{m}}{\rho_{x}} .
$$

Nesta classe de modelos existe liberdade para escolher a função $f$. Asssim, por exemplo, Vargas et al. (2012) fizeram a seguinte escolha:

$$
f(a)=1+c a^{5} e^{-a^{2} / \sigma^{2}}
$$

onde $c$ e $\sigma$ são constantes. Neste caso a densidade de energia escura é dada por:

$$
\rho_{x}=\rho_{x 0}-c \rho_{m 0} e^{-a^{2} / \sigma^{2}}\left(a^{2}-\frac{3}{2} \sigma^{2}\right)
$$

\subsubsection{Modelos com $\Lambda(a)$}

Neste tipo de modelo considera-se que a constante cosmológica é uma função do fator de escala, existem vários modelos deste tipo, nesta seção vamos considerar o caso geral e no próximo capítulo 6 vamos estudar um caso específico.

Para esta classe de modelos a taxa de transferência de energia é obtida através da equação (5.7):

$$
Q=-\frac{1}{8 \pi G} \frac{d \Lambda}{d t}
$$

Substituindo em (5.8) e transformando as derivadas temporais para derivadas em termos do fator de escala temos

$$
\frac{1}{a^{3}} \frac{d}{d a} \rho_{m} a^{3}=-\frac{1}{8 \pi G} \frac{d \Lambda}{d a}
$$

Vemos que se a expressão para $\Lambda(a)$ é conhecida, podemos obter a densidade de energia da matéria escura e com isso o parâmetro de Hubble. Integrando (5.35) por partes obtemos

$$
\rho_{m}(a)=\left(\rho_{m 0}+\frac{\Lambda\left(a_{0}\right)}{8 \pi G}\right)\left(\frac{a_{0}}{a}\right)^{3}-\frac{\Lambda(a)}{8 \pi G}+\frac{3}{8 \pi G a^{3}} \int_{a_{0}}^{a} a^{2} \Lambda(a) d a .
$$

Portanto, ao se estabelecer $\Lambda(a)$ obtém-se a densidade da matéria e todas as quantidades cosmológicas de interesse. Como exemplo vamos considerar a seguinte forma funcional:

$$
\Lambda(a)=\Lambda_{0}+\gamma a^{-n}
$$


onde $n$ é um número natural. Substituindo em (5.36) e integrando obtemos

$$
\rho_{m}=\left(\rho_{m 0}-\frac{n \gamma a_{0}^{-n}}{8 \pi G(3-n)}\right)\left(\frac{a_{0}}{a}\right)^{3}+\frac{n \gamma a^{-n}}{8 \pi G(3-n)} .
$$

A expressão usual para a densidade da matéria é modificada por dois fatores, primeiro a um termo a mais que escala com $a^{-3}$ e surge um segundo fator que escala com $a^{-n}$, a mesma dependência que ocorre em $\Lambda$. 


\section{Capítulo 6}

\section{Tensão SNe-CMB e o Decaimento do Vácuo}

Após discutir a cosmologia padrão e modelos com interação, apresentamos neste capítulo um modelo cuja principal motivação é resolver a tensão entre os dados de SNe e CMB. Primeiramente vamos apresentar um histórico deste modelo, discutir a tensão SNe-CMB e por fim fazer os testes observacionais. É neste capítulo que se encontra a parte original da dissertação. Nosso modelo é uma variante do cenário com decaimento do vácuo originalmente proposto por Chen e Wu (1990).

\subsection{Histórico}

Nesta seção vamos apresentar alguns artigos que estabeleceram a lei de decaimento utilizada neste trabalho bem como algumas propostas similares ou relacionadas. Iniciaremos com o trabalho original de Ozer e Taha que iniciou, em meados dos anos 80 (antes da descoberta da aceleração do universo) a discussão sobre modelos com $\Lambda(t)$.

\subsubsection{Modelo de Ozer e Taha}

A origem do modelo de interacão proposto neste trabalho foi inicialmente discutida por Ozer e Taha em 1986 (Ozer e Taha, 1986, 1987). Esses autores propuseram a seguinte lei fenomenológica para o decaimento de $\Lambda$ :

$$
\Lambda(a)=\frac{3}{a^{2}}
$$

Para obter esta lei de decaimento eles inicialmente consideraram um universo com curvatura arbitrária e impuseram que a soma das densidades de energia da matéria e radiação é sempre igual a densidade crítica. Além disso, impondo a condição de que a 
variação de entropia é positiva foi possível determinar que a curvatura também é positiva.

Começando com a equação de Friedmann com constante cosmológica e curvatura:

$$
\frac{\dot{a}^{2}}{a^{2}}=\frac{8 \pi G}{3} \rho+\frac{\Lambda}{3}-\frac{\kappa}{a^{2}}
$$

substituindo $\rho$ pela densidade crítica

$$
\rho_{c} \equiv \frac{3 H^{2}}{8 \pi G}=\frac{3}{8 \pi G} \frac{\dot{a}^{2}}{a^{2}}
$$

e isolando $\Lambda$ obtém-se

$$
\Lambda=\frac{3 \kappa}{a^{2}}
$$

A equação para a entropia é:

$$
T d S \equiv d(\rho V)+p d V=-\frac{V}{8 \pi G} d \Lambda
$$

esta equação é obtida a partir da equação de conservação pois $V \propto a^{3}$, logo se a variação da entropia deve ser positiva implica que a variação de $\Lambda$ é negativa, ou seja, há decaimento, com isso a curvatura é fixada em $\kappa=1$, desta forma (6.4) se reduz à (6.1).

A constante cosmológica na descrição de Ozer e Taha decai em radiação (não em matéria escura), portanto, a densidade de energia da radiação é modificada (Ozer e Taha, 1987)

$$
\rho_{r}=\frac{3}{8 \pi G a^{2}}\left(1-\frac{a_{i}^{2}}{a^{2}}\right)
$$

onde $a_{i}$ é o fator de escala no instante inicial, definido como o tempo em que $\rho_{r i}=0$. Neste modelo não há singularidade inicial e todas as funções cosmológicas são finitas em $t=0$. A dependência do fator de escala com o tempo é dada por:

$$
a^{2}=a_{i}^{2}+t^{2}
$$

esta relação é obtida ao se resolver a equação de conservação impondo $\rho=\rho_{\text {crit }}$. Por não ter singularidade inicial não há problema de horizonte (é possível mostrar que todo o universo é casualmente conectado desde o início), o problema dos monopólos magnéticos também é resolvido. 
O modelo de Ozer e Taha pode ser comparado com o modelo cosmológico de Einstein (seção 2.4.1). A expressão que Einstein obteu para a constante cosmológica (2.46) é semelhante a expressão de Ozer e Taha (6.1) e em ambos os modelos o universo é fechado, a diferença é que Einstein considerou um universo estático, então sua constante cosmológica é realmente constante, pode-se pensar que o modelo de Ozer e Taha é uma generalização do modelo de Einstein.

\subsubsection{Modelo de Chen e $\mathrm{Wu}$}

Alguns anos depois do modelo de Ozer e Taha, Chen e Wu (1990) apresentaram um argumento adimensional para a lei $a^{-2}$. Basicamente eles argumentaram que uma expressão natural para o decaimento é uma quantidade adimensional vezes a densidade de Planck (que fixa a escala de energia):

$$
\Lambda(a) \propto \frac{\rho_{p l}}{8 \pi G}\left(\frac{r_{p l}}{a}\right)^{n},
$$

onde a densidade e o comprimento de Planck são dados por:

$$
\rho_{p l}=\frac{c^{5}}{\hbar G^{2}}
$$

$\mathrm{e}$

$$
L_{p l}=\left(\frac{\hbar G}{c^{3}}\right)^{1 / 2} .
$$

A dependência temporal é dada através do fator de escala. Como a relatividade geral é uma teoria clássica, não se espera um fator $\hbar$ nas equações cosmológicas. Sendo assim, para se cancelar a constante de Planck toma-se $n=2$, resultando em:

$$
\Lambda=\frac{\gamma}{a^{2}}
$$

onde $\gamma$ é um parâmetro adimensional. Um argumento similar a este é utilizado para determinar a energia de ponto zero do átomo de hidrogênio. De fato, por questões dimensionais conclui-se que esta energia pode ser escrita como o produto da energia de repouso do elétron com uma potência da constante de estrutura fina

$$
E=m c^{2}\left(\frac{e^{2}}{\hbar c}\right)^{p} .
$$


Assim, como o átomo de hidrogênio é não relativístico conclui-se que $p=2$ para eliminar a velocidade da luz (Sakurai, 1993).

A partir dai Chen e Wu exploram as consequências de seu modelo, a equação de conservação é resolvida para a radiação $(p=\rho / 3)$ e matéria $(p=0)$ separadamente:

$$
\rho_{r}=A_{1} a^{-4}+\frac{\gamma}{8 \pi G a^{2}}
$$

e

$$
\rho_{m}=A_{2} a^{-3}+\frac{2 \gamma}{8 \pi G a^{2}}
$$

onde $A_{1}$ e $A_{2}$ são constantes positivas. Apesar da dependência temporal da constante cosmológica ser a mesma em Ozer e Taha e em Chen e Wu, os modelos são bem diferentes, enquanto Ozer e Taha impõe que a densidade total é igual a densidade crítica para obter a forma do decaimento e a curvatura, Chen e Wu partem de argumentos dimensionais e deixam a curvatura livre.

\subsubsection{Modelo de Abdel-Rahman}

Outro trabalho usando a mesma lei de decaimento foi publicado por Abdel-Rahman em 1992. O autor generalizou o tratamento de Ozer e Taha ao abandonar a hipótese de que $\rho=\rho_{\text {crit }}$ e para o decaimento de $\Lambda(t)$ considerou a mesma expressão de Chen e Wu (o fator 3 foi introduzido por conveniência matemática)

$$
\Lambda=\frac{3 \gamma}{a^{2}}
$$

Neste modelo também não há singularidade inicial, sendo que o tempo $t=0$ é definido quando o fator de escala é mínimo, ou seja, quando $\dot{a}=0$. Usando esta condição nas equações de Friedmann pode-se obter o valor da densidade no tempo inicial

$$
\frac{8 \pi G}{3} \rho_{0} a_{0}^{2}=\kappa-\gamma
$$

onde 0 denota os valores em $t=0$. Para $\rho_{0} \geq 0$ a curvatura deve ser positiva. Para decaimento em radiação o resultado para a densidade é dado por

$$
\rho_{r}=\frac{3 \gamma}{8 \pi G a^{2}}\left[1-\frac{(2 \gamma-1) a_{0}^{2}}{\gamma a^{2}}\right]
$$


onde $\rho_{0}$ foi eliminado usando (6.16), com $\kappa=1$. Comparando (6.17) com a expressão análoga de Ozer e Taha (6.6) percebe-se que são idênticas quando $\gamma=1$, comparando com a expressão de Chen e Wu (6.13) também há uma identificação caso $A_{1}=\frac{3 a_{0}^{2}(2 / 3 \gamma-1)}{8 \pi G}$, lembrando que o $\gamma$ de Chen e Wu é o triplo do valor de Abdel-Rahman.

\subsubsection{Modelo de Carvalho, Lima e Waga}

Para finalizar esta seção apresentaremos uma modificação do modelo de Chen e Wu proposta por Carvalho, Lima e Waga (1992), onde a lei de decaimento é:

$$
\Lambda=3 \beta H^{2}+\frac{3 \alpha}{a^{2}}
$$

onde $\alpha$ e $\beta$ são constantes adimensionais e os fatores 3 foram também introduzidos por conveniência matemática. O argumento por trás da introdução do primeiro termo em (6.18) é similar ao de Chen e Wu, como o parâmetro de Hubble é dado por $\dot{a} / a$ então existe a possibilidade de $\Lambda$ depender de $H^{2}$.

\subsubsection{Modelo de Lopez e Nanopoulos}

Neste modelo Lopez e Nanopoulos (1996) usam um ansazt similar ao de Chen e Wu, a diferença é que a variação de $\Lambda$ é dada em função do tempo, não do fator de escala

$$
\Lambda=\frac{\Lambda_{p l}}{\left(t / t_{p l}\right)^{2}} \propto \frac{1}{t^{2}}
$$

o que implica que o fator de escala deve ser proporcional ao tempo.

\subsubsection{Modelo de Chen e Wu Generalizado}

Por fim vamos apresentar outra generalização de Chen e Wu, feita por John e Joseph (2000). Neste artigo os autores argumentam que o ansatz dimensional de Chen e Wu deve ser feito para a densidade de energia total, não somente para a do vácuo, com isso:

$$
\widetilde{\rho} \propto \frac{1}{a^{2}}
$$

onde:

$$
\widetilde{\rho}=\rho_{m}+\rho_{\Lambda}
$$


Na notação do artigo $m$ indica tanto matéria relativística quanto não relativística $\left(p_{m}=\right.$ $\left.\omega \rho_{m}\right)$. A equação de conservação (5.6) neste caso é dada por

$$
\widetilde{\rho}+3 \widetilde{p}=0
$$

pois:

$$
\dot{\widetilde{\rho}}=-2 \frac{\dot{a}}{a} \widetilde{\rho}
$$

onde $\widetilde{p}$ é definido de maneira idêntica à $\widetilde{\rho}$. Usando (6.21) em (6.22):

$$
\frac{\rho_{m}}{\rho_{\Lambda}}=\frac{2}{1+3 \omega},
$$

ou seja, as densidades de matéria e vácuo devem ter valores comparáveis. Substituindo (6.20) na primeira equação de Friedmann (2.4) obtém-se que $a$ é proporcional ao tempo

$$
a(t)=m t
$$

onde $m$ é a constante de proporcionalidade. A densidade de energia total é:

$$
\widetilde{\rho}=\frac{3}{8 \pi G} \frac{m^{2}+\kappa}{a^{2}}
$$

neste modelo também não há problema de horizonte ou de planura.

\subsubsection{Análise Termodinâmica}

Usando a teoria de Landau-Lifshitz 1 para flutuações fora do equilibrio, Pavón (1991) analisou alguns modelos de decaimento, incluindo o de Ozer e Taha bem como de Chen e Wu, para verificar quais eram termodinamicamente viáveis. Considerando que há decaimento em radiação Pavón conclui que os modelos com $\Lambda \propto a^{-2}$ são termodinamicamente consistentes.

\footnotetext{
${ }^{1}$ Landau e Lifshitz (1984), capítulo 17.
} 


\subsection{Motivações}

\subsubsection{Termo Constante}

Neste trabalho vamos utilizar uma deneralização da lei de decaimento obtida por Ozer e Taha:

$$
\Lambda=\Lambda_{0}+\frac{3 \alpha}{a^{2}}
$$

onde $\alpha$ é um parâmetro constante. A diferença entre esta lei e a de Chen e Wu é o termo constante $\Lambda_{0}$. Para justificar a introdução deste termo vamos primeiro calcular o parâmetro de desaceleração para um modelo sem o termo $\Lambda_{0}$. Conforme será mostrado posteriormente (equação (6.56)), usando as definições (6.53) e (6.55)), a desaceleração é dada por $\left(\operatorname{com} \Lambda_{0}=0\right)$ :

$$
q=\frac{\left(\Omega_{m 0}-2 \Omega_{\alpha 0}\right)(1+z)^{3}+\Omega_{\alpha 0}(1+z)^{2}}{\left(\Omega_{m 0}-2 \Omega_{\alpha 0}\right)(1+z)^{3}+\left(1-\Omega_{m 0}+2 \Omega_{\alpha 0}\right)(1+z)^{2}},
$$

onde os parâmetros $\Omega_{m 0}$ e $\Omega_{\alpha 0}$ estão definidos em (6.39) e (6.41). Mais adiante será mostrado que $\Omega_{m 0}-2 \Omega_{\alpha 0}$ é sempre positivo, então o parâmetro de desaceleração também é positivo para todos os redshifts, o que está em desacordo com os dados de SNe que indicam que o universo está acelerado, ou seja, q é menor que zero. Para obter esta transição entre desaceleração e aceleração pode-se introduzir o termo constante em (6.27).

Por outro lado, com a adoção do termo constante pode-se recuperar facilmente o modelo $\Lambda C D M$, basta ter $\alpha=0$, algo que não ocorre nos outros modelos discutidos acima.

\subsubsection{Tensão $\mathrm{SNe}-\mathrm{CMB}$}

Os dados de supernovas e da radiação cósmica de fundo são ambos sensíveis aos parâmetros de densidade da energia e da matéria escura, porém, comparando os contornos de confiança dos dados (figura 3.7), percebe-se que são bem diferentes. De fato, os dados de CMB preferem um universo próximo ao plano, enquanto SNe favorece um universo fechado. Ao se fazer a análise conjunta dos dados o espaço de parâmetros é bastante limitado e se obtém o modelo de concordância cósmica, por outro lado, seria interessante analisar se estas diferenças são realmente apenas uma coincidência ou se existe alguma razão física para a tensão. 
Conforme discutido no capítulo anterior, é possível que exista o decaimento de $\Lambda$, se este decaimento for do tipo Chen e Wu com adição de um termo constante obteremos:

1. Um termo que escala exatamente como a curvatura nas equações de Friedmann.

2. Uma correção ao atual modelo de concordância cósmica que deverá ser limitada pelas observações.

Por outro lado, sabemos que os modelos inflacionários preveem um universo plano (Kolb e Turner, 1990) e os dados de CMB são obtidos a altos redshift enquanto os de SNe a baixo redshift. Deste ponto de vista pode-se considerar que a curvatura que as supernovas indicam nada mais é do que o efeito do decaimento do vácuo, sendo esperado que CMB meça um universo plano pois a altos redshifts pode-se desprezar a contribuição da variação da constante cosmológica. Além disso, o modelo padrão pode ser aproximadamente mantido por conta da presença do termo constante, $\Lambda_{0}$.

É importante mencionar que existem outras tentativas de explicação da tensão SNeCMB. Amendola et al. (2010), por exemplo, consideram técnicas numéricas em lentes fracas para tentar resolver esse problema enquanto Shafieloo et al. (2009) discutiram o problema variando a equação de estado da energia escura (e.g. equação (4.21)). Mais recentemente, Lima et al. (2013) discutiram como inomogeinedades parametrizadas através da equação de Dyer-Roeder resolveriam este problema.

O recente resultado do satélite Planck (Ade et al., 2013) também apresenta uma certa tensão com dados de SNe. A figura 6.1 mostra a distribuição posterior de $\Omega_{m 0}$ do Planck comparado com resultados de SNe. Do gráfico percebe-se que existe uma certa tensão entre os dados do Planck com a compilação de SNe SNLS (Conley et al.,, 2011). As análises de SNe feitas pela equipe do Planck foram restritas ao modelo $\Lambda C D M$ plano, isto explica a apresentação da distribuição de probabilidade de um parâmetro e não contornos de confiança.

\subsection{Novo Modelo: $\Lambda_{0}+3 \alpha a^{-2}$}

Nas seções anteriores apresentamos as motivações e o histórico do modelo a ser estudado, nesta seção inicialmente obteremos todas as equações e parâmetros do modelo 


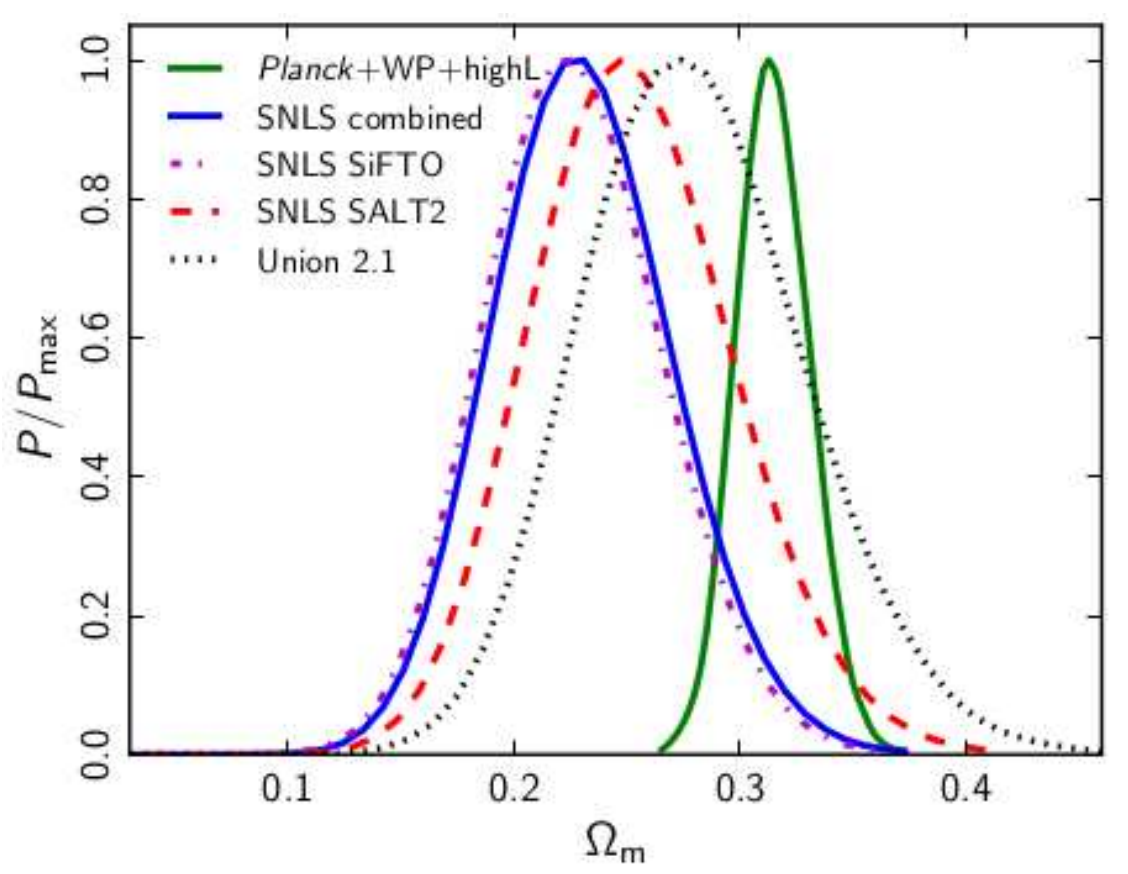

Figura 6.1: Distribuição posterior de $\Omega_{m 0}$ de dados do Planck e de SNe, gráfico retirado de Ade et al. (2013).

considerando uma curvatura arbitrária. Posteriormente nos concentraremos no caso de curvatura nula $\left(\Omega_{\kappa}=0\right)$, cuja tensão SNe-CMB é o problema central de nosso trabalho.

\subsubsection{Densidade da Matéria Escura e Parâmetro de Hubble}

Considerando que $\Lambda(t)$ obedece a seguinte lei de decaimento:

$$
\Lambda=\Lambda_{0}+\frac{3 \alpha}{a^{2}}
$$

onde o fator 3 foi introduzido por conveniência matemática. Note que esta lei de decaimento é similar a usada por Waga (1993), a diferença sendo que nossa expressão não possui um termo que escala como $H^{2}$. Escrevendo $\Lambda$ como a densidade do vácuo temos que

$$
\rho_{v}=\frac{\Lambda_{0}}{8 \pi G}+\frac{3 \alpha}{8 \pi G a^{2}} \equiv \rho_{\Lambda 0}+\rho_{\alpha}
$$

onde definimos

$$
\rho_{\Lambda 0}=\frac{\Lambda_{0}}{8 \pi G} \text { e } \rho_{\alpha}=\frac{3 \alpha}{8 \pi G a^{2}}
$$

e para facilitar a notação chamaremos a parte constante de densidade do vácuo, embora a densidade total do vácuo seja $\rho_{\Lambda 0}+\rho_{\alpha 0}$. Como foi visto, com as equações (5.7) e (5.8) 
podemos obter a densidade de energia da matéria escura:

$$
\dot{\rho}_{m}+3 \frac{\dot{a}}{a} \rho_{m}=-\dot{\rho}_{v}=-\dot{\rho}_{\alpha}
$$

onde de (6.31) temos

$$
\dot{\rho}_{\alpha}=\frac{3 \alpha \dot{a}}{a^{3} 4 \pi G}
$$

A equação (6.32) pode ser reescrita como

$$
\frac{1}{a^{3}} \frac{d}{d t} a^{3} \rho_{m}=\frac{1}{a^{3}} \frac{3 \alpha}{4 \pi G} \frac{d a}{d t}
$$

cuja integral é imediata

$$
\rho_{m}=\frac{B}{a^{3}}+\frac{3 \alpha}{4 \pi G a^{2}},
$$

onde B é uma constante que pode ser fixada tomando $\rho_{m 0}=\rho_{m}\left(a_{0}\right)$. É fácil verificar que a densidade de matéria toma a seguinte forma:

$$
\rho_{m}=\rho_{m 0}\left(\frac{a_{0}}{a}\right)^{3}\left[1-\frac{3 \alpha}{4 \pi G \rho_{m 0} a_{0}^{2}}\right]+\frac{3 \alpha}{4 \pi G a_{0}^{2}}\left(\frac{a_{0}}{a}\right)^{2} .
$$

Podemos ver que o caso padrão é modificado por dois termos e como seria esperado, para $\alpha=0$ o modelo $\Lambda C D M$ é recuperado. Considerando a primeira equação de Friedmann (2.4) podemos obter o parâmetro de Hubble2

$$
\frac{H^{2}}{H_{0}^{2}}=\frac{8 \pi G \rho_{m}}{3 H_{0}^{2}}+\frac{\Lambda_{0}}{3 H_{0}^{2}}+\frac{\alpha}{a^{2} H_{0}^{2}}-\frac{\kappa}{a_{0}^{2} H_{0}^{2}} \frac{a_{0}^{2}}{a^{2}} .
$$

Para $H=H_{0}$ segue que

$$
\Omega_{m 0}+\Omega_{\Lambda 0}+\Omega_{\alpha 0}+\Omega_{\kappa 0}=1
$$

onde os parâmetros de densidade foram definidos da maneira usual:

$$
\Omega_{m 0} \equiv \frac{8 \pi G \rho_{m 0}}{3 H_{0}^{2}}
$$

\footnotetext{
${ }^{2} \mathrm{Na}$ nossa análise não vamos incluir a densidade de energia dos bárions pois sua influência é de apenas um termo aditivo em $\Omega_{m 0}$, além do que, por ter um valor pequeno (cerca de 0,04 ), sua inclusão não afetaria significativamente nossos resultados e conclusões.
} 


$$
\begin{gathered}
\Omega_{\Lambda 0} \equiv \frac{\Lambda_{0}}{3 H_{0}^{2}}, \\
\Omega_{\alpha 0} \equiv \frac{\alpha}{a_{0}^{2} H_{0}^{2}},
\end{gathered}
$$

$\mathrm{e}$

$$
\Omega_{\kappa 0} \equiv-\frac{\kappa}{a_{0}^{2} H_{0}^{2}}
$$

Para comparar com as observações é interessante reescrever $\rho_{m}$ e $H$ em termos dos $\Omega$ 's:

$$
\begin{gathered}
\rho_{m}=\rho_{m 0}\left[\left(1-\frac{2 \Omega_{\alpha 0}}{\Omega_{m 0}}\right)\left(\frac{a_{0}}{a}\right)^{3}+\frac{2 \Omega_{\alpha 0}}{\Omega_{m 0}}\left(\frac{a_{0}}{a}\right)^{2}\right], \\
\frac{H^{2}}{H_{0}^{2}}=\left(\Omega_{m 0}-2 \Omega_{\alpha 0}\right)\left(\frac{a_{0}}{a}\right)^{3}+\Omega_{\Lambda 0}+3 \Omega_{\alpha 0}\left(\frac{a_{0}}{a}\right)^{2}+\Omega_{\kappa 0}\left(\frac{a_{0}}{a}\right)^{2} .
\end{gathered}
$$

Impondo que $\rho_{m} \geq 0$ (a condição de energia fraca) ao longo da evolução cósmica obtemos o seguinte vínculo:

$$
\frac{2 \Omega_{\alpha 0}}{\Omega_{m 0}} \geq 1
$$

supondo que $\alpha$ é positivo. Esta condição pode ser reescrita como

$$
0 \leq \Omega_{\alpha 0} \leq \frac{\Omega_{m 0}}{2}
$$

ou ainda

$$
0 \leq \alpha<\frac{a_{0}^{2} H_{0}^{2} \Omega_{m 0}}{2}
$$

A condição acima implica na existência de um limite na interação energia-matéria escura. Note que tal limite não existe para $\alpha<0$. Eliminando $\Omega_{\kappa 0}$ da expressão (6.44) obtemos (ver (6.38) $)$

$$
\frac{H^{2}}{H_{0}^{2}}=\left(\Omega_{m 0}-2 \Omega_{\alpha 0}\right)\left(\frac{a_{0}}{a}\right)^{3}+\Omega_{\Lambda 0}+\left(1-\Omega_{m 0}-\Omega_{\Lambda 0}+2 \Omega_{\alpha 0}\right)\left(\frac{a_{0}}{a}\right)^{2},
$$

vemos que novamente o modelo $\Lambda C D M$ padrão é facilmente recuperado quando $\alpha=0$. De (6.44) pode-se perceber que o decaimento simula um termo de curvatura que depende 
do sinal de $\alpha$. Na equação (6.48) as densidades $\Omega_{m 0}$ e $\Omega_{\alpha 0}$ aparecem degeneradas, ou seja, o parâmetro de Hubble depende somente de uma combinação delas:

$$
\Omega_{m e f 0} \equiv \Omega_{m 0}-2 \Omega_{\alpha 0}
$$

Se $\alpha$ é positivo, temos que $\Omega_{m e f 0}$ é sempre positivo pela condição (6.46). Na equação (6.48) vemos que $\Omega_{m e f 0}$ desempenha o papel de parâmetro de densidade efetivo da matéria escura. De maneira similar, a quantidade definida como

$$
\Omega_{\kappa e f 0} \equiv 1-\Omega_{m e f 0}-\Omega_{\Lambda 0}=\Omega_{\kappa 0}+3 \Omega_{\alpha 0},
$$

se comporta como uma curvatura efetiva. As equações deste modelo são idênticas as de $\Lambda C D M$ com $\Omega_{m 0}$ substituido por $\Omega_{m e f 0}$ e $\Omega_{\kappa 0}$ por $\Omega_{\kappa e f 0}$.

\subsubsection{Parâmetros de Densidade de Desaceleração}

Para visualizar a evolução das quantidades cosmológicas define-se os parâmetros de densidade dependentes do redshift:

$$
\Omega_{m}(z) \equiv \frac{8 \pi G \rho_{m}}{3 H_{0}^{2}} \frac{H_{0}^{2}}{H^{2}}
$$

$\mathrm{e}$

$$
\Omega_{v}(z) \equiv \frac{\Lambda_{0}}{3 H_{0}^{2}} \frac{H_{0}^{2}}{H^{2}}+\frac{\alpha}{a_{0}^{2} H_{0}^{2}} \frac{H_{0}^{2} a_{0}^{2}}{H^{2} a^{2}} \equiv \Omega_{\Lambda}(z)+\Omega_{\alpha}(z),
$$

substituindo (6.43) e (6.48):

$$
\begin{aligned}
& \Omega_{m}=\frac{\left(\Omega_{m 0}-2 \Omega_{\alpha 0}\right)(1+z)^{3}+2 \Omega_{\alpha 0}(1+z)^{2}}{\left(\Omega_{m 0}-2 \Omega_{\alpha 0}\right)(1+z)^{3}+\Omega_{\Lambda 0}+\left(1-\Omega_{m 0}+2 \Omega_{\alpha 0}-\Omega_{\Lambda 0}\right)(1+z)^{2}} \\
& \Omega_{\Lambda}=\frac{\Omega_{\Lambda 0}}{\left(\Omega_{m 0}-2 \Omega_{\alpha 0}\right)(1+z)^{3}+\Omega_{\Lambda 0}+\left(1-\Omega_{m 0}+2 \Omega_{\alpha 0}-\Omega_{\Lambda 0}\right)(1+z)^{2}}
\end{aligned}
$$

$\mathrm{e}$

$$
\Omega_{\alpha}=\frac{\Omega_{\alpha 0}(1+z)^{2}}{\left(\Omega_{m 0}-2 \Omega_{\alpha 0}\right)(1+z)^{3}+\Omega_{\Lambda 0}+\left(1-\Omega_{m 0}+2 \Omega_{\alpha 0}-\Omega_{\Lambda 0}\right)(1+z)^{2}} .
$$




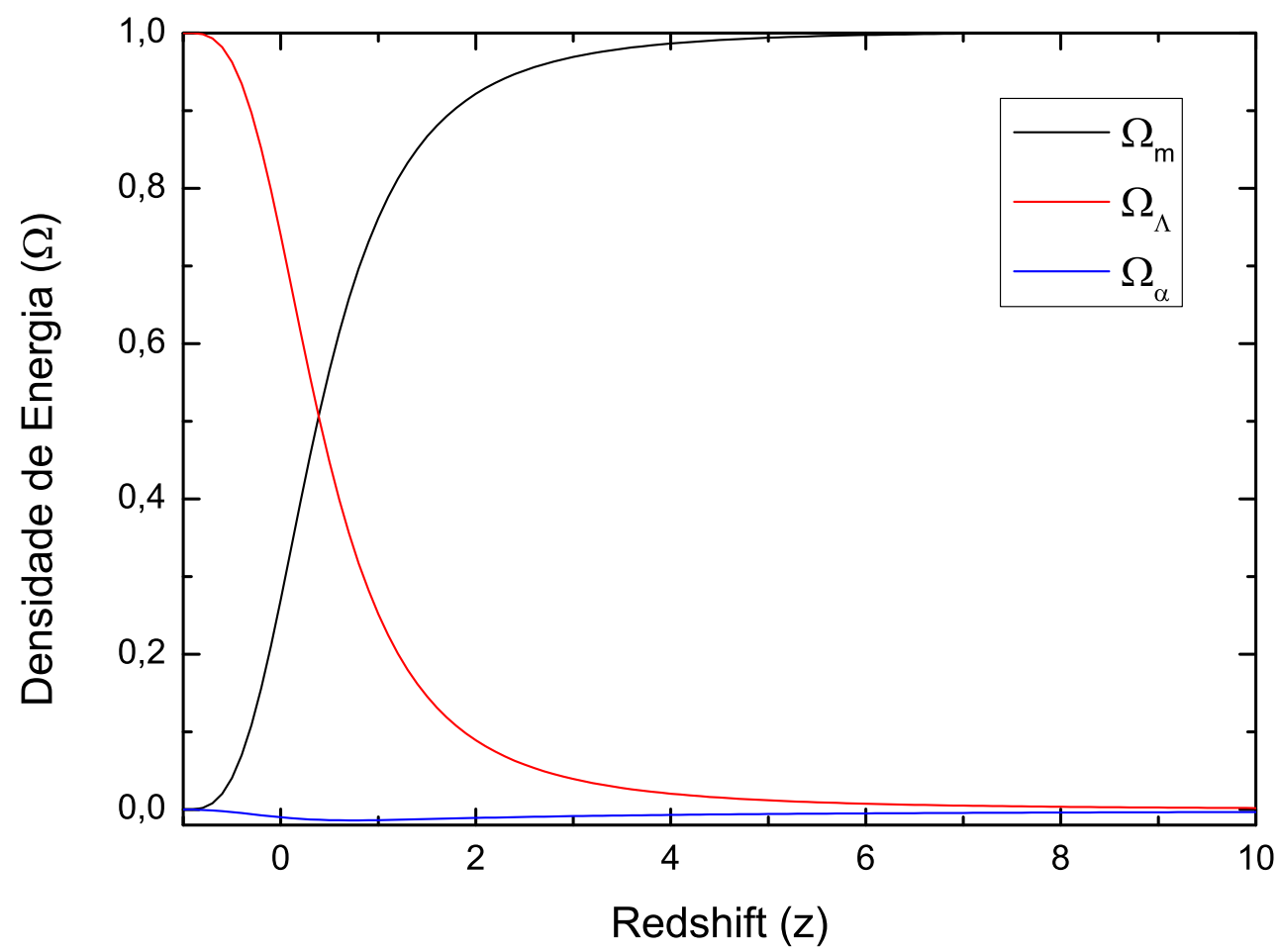

Figura 6.2: Parâmetros de densidade em função do redshift, para $\Omega_{m 0}=0,27, \Omega_{\Lambda 0}=0,74$ e $\Omega_{\alpha 0}=-0,01$.

A figura 6.2 mostra a evolução cósmica das densidades para os melhores valores dos parâmetros obtidos com o teste de $\chi^{2}$ (ver seção 6.4).

Outra quantidade de interesse é o parâmetro de desaceleração (ver equação (2.15) ). Da segunda equação de Friedmann (2.5) obtemos:

$$
q=\frac{\Omega_{m}}{2}-\Omega_{\Lambda}-\Omega_{\alpha}
$$

Finalmente justificando a expressão (6.28), onde $\Omega_{\Lambda 0}=0$. Observe que o parâmetro de desaceleração é nulo no redshift de transição $\left(z_{T}\right)$, dado por:

$$
z_{T}=\left(\frac{2 \Omega_{\Lambda 0}}{\Omega_{m 0}-2 \Omega_{\alpha 0}}\right)^{1 / 3}-1 .
$$

Vemos que a expressão acima é idêntica do modelo $\Lambda C D M$ para $\Omega_{\kappa 0}=0$ (ver equação $(4.9)$ ).

No nosso modelo não há retorno a desaceleração, como ocorre com outras leis de de- 


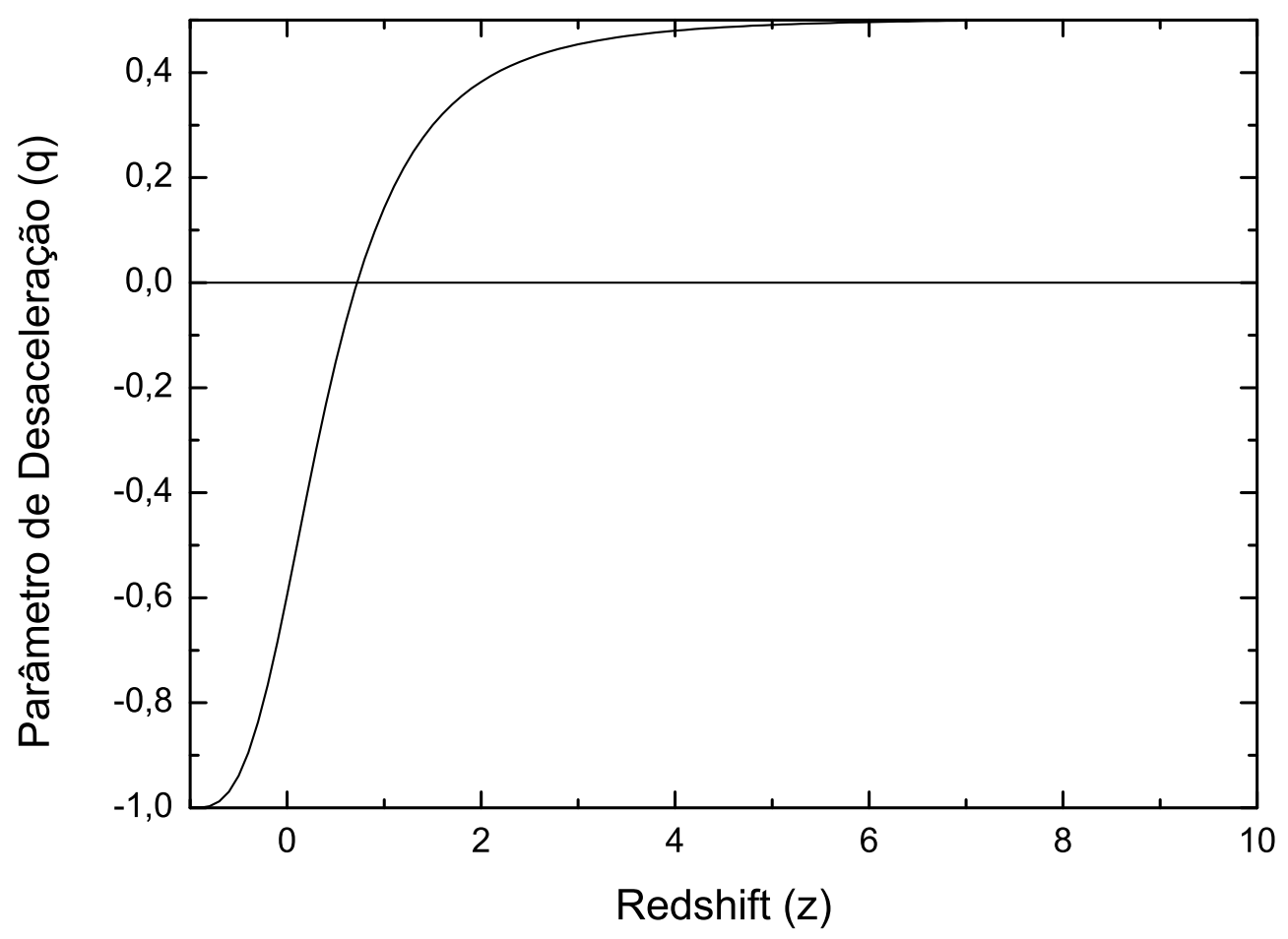

Figura 6.3: Parâmetro de desaceleração em função do redshift, para $\Omega_{m 0}=0,27, \Omega_{\Lambda 0}=0,74$ e $\Omega_{\alpha 0}=-0,01$.

caimento (ver, por exemplo, Carvalho et al. 2006). Em Guimarães e Lima (2011) há uma abordagem cosmográfica (independente de modelos) onde a possibilidade de desaceleração no futuro é investigada. No futuro, nosso modelo se assemelha a $\Lambda C D M$, pois ambos serão dominados apenas pela constante cosmológica.

Por fim, pode-se calcular a idade do universo neste modelo através da equação (2.25). Substituindo (6.48), temos

$$
H_{0} t_{0}=\int_{0}^{1} \frac{d x}{x \sqrt{\left(\Omega_{m 0}-2 \Omega_{\alpha 0}\right) x^{-3}+\Omega_{\Lambda 0}+\left(1-\Omega_{m 0}+2 \Omega_{\alpha 0}-\Omega_{\Lambda 0}\right) x^{-2}}} .
$$

Como seria esperado, a expressão acima se reduz a idade do modelo $\Lambda C D M$ (curvatura arbitrária) no caso limite, $\Omega_{\alpha 0}=0$. 


\subsubsection{Caso Plano}

Para finalizar esta seção discutiremos agora o caso plano, para $\Omega_{\kappa}=0$, a equação (6.38) que relaciona as densidades de energia das componente se reduz para

$$
\Omega_{m 0}+\Omega_{\Lambda 0}+\Omega_{\alpha 0}=1
$$

Assim, vemos que agora é possível eliminar uma das densidades e ficar com apenas dois parâmetros livres. A densidade da matéria escura e a curvatura efetiva (equações (6.49) e (6.50) ) passam a ser (após eliminar $\Omega_{\alpha 0}$ )

$$
\Omega_{m e f 0}=3 \Omega_{m 0}+2\left(\Omega_{\Lambda 0}-1\right)
$$

e

$$
\Omega_{k e f 0}=3\left(1-\Omega_{m 0}-\Omega_{\Lambda 0}\right)
$$

portanto, mesmo para o caso plano ainda existe uma curvatura efetiva. Podemos dividir o espaço de parâmetros $\Omega_{m 0}-\Omega_{\Lambda 0}$ em duas regiões distintas, acima (abaixo) da linha $\Omega_{m 0}+$ $\Omega_{\Lambda 0}=1$ temos a região onde $\Omega_{\alpha 0}$ é negativo (positivo). Na região positiva ocorre uma área proibida, advinda da combinação da desigualdade (6.46) com a condição de planura (6.59), cuja inequação é dada por

$$
\Omega_{m 0} \geq \frac{2}{3}\left(1-\Omega_{\Lambda 0}\right)
$$

O gráfico 6.4 ilustra estas regiões.

\subsubsection{Idade no Modelo Plano}

É interessante calcular a idade no modelo plano e comparar com $\Lambda C D M$. O gráfico 6.5 mostra o parâmetro de idade $\left(H_{0} t_{0}\right)$ em função de $\Omega_{\Lambda 0}$ para três valores diferentes de $\Omega_{\alpha 0}$. Para $\Omega_{\alpha 0}$ positivo a idade aumenta em relação ao modelo $\Lambda C D M\left(\Omega_{\alpha 0}=0\right)$, conforme o esperado. No caso negativo ocorre o inverso.

Para obter as demais equações, como os parâmetros de Hubble e de desaceleração, basta substituir os novos valores de $\Omega_{m e f 0}$ e $\Omega_{\kappa e f 0}$. 


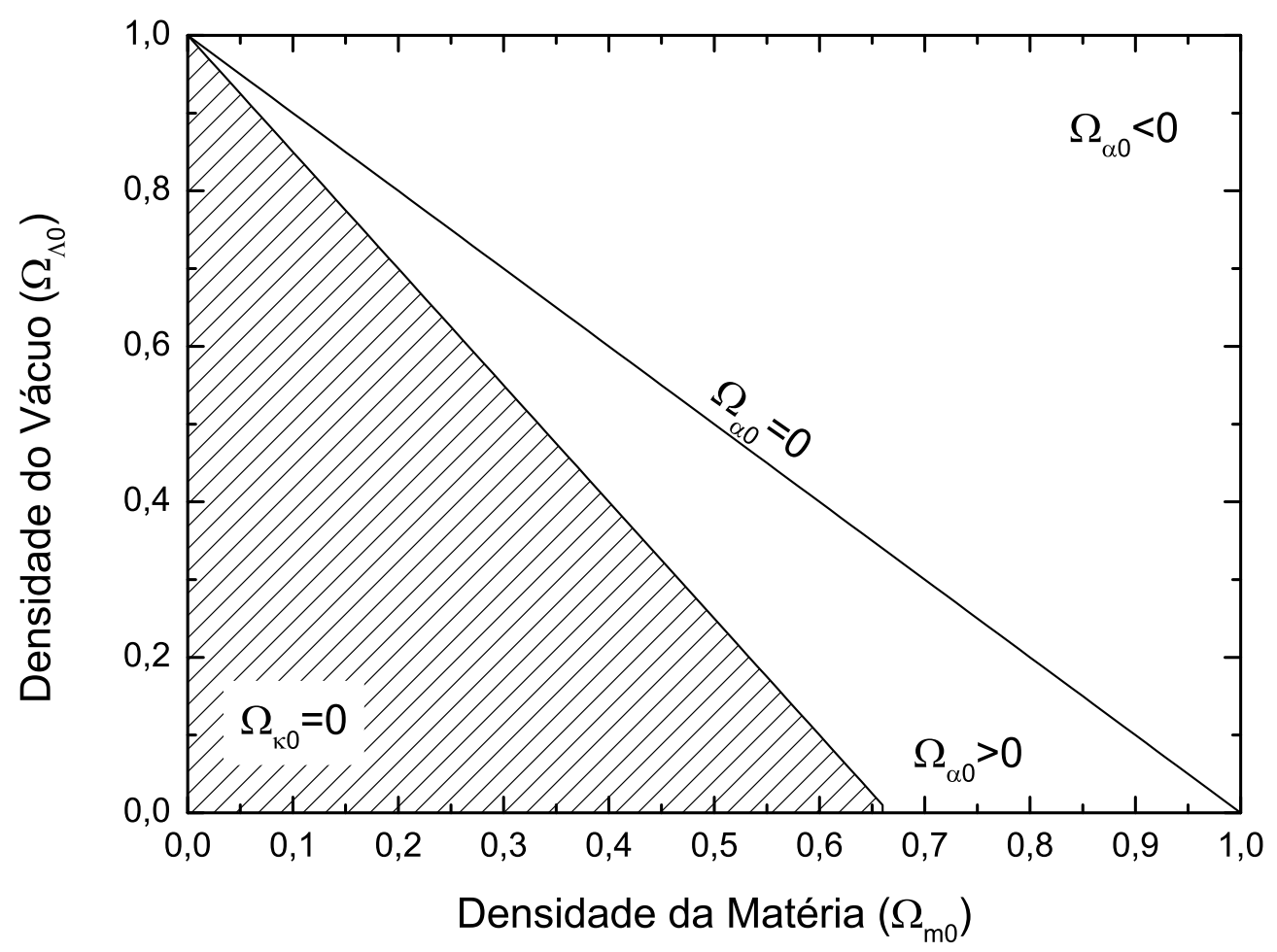

Figura 6.4: Divisão do espaço de parâmetro $\Omega_{\Lambda 0}$ e $\Omega_{m 0}$. A região hachurada é proibida de acordo com a desigualdade (6.62).

\subsection{Testes Observacionais}

Nesta seção aplicaremos os testes observacionais discutidos na seção 3.2, que são SNe, CMB e BAO. Para obter o melhor ajuste para os parâmetros e os contornos de confiança vamos utilizar o teste do $\chi^{2}$, que é uma máxima verossimilhança, nesta seção primeiramente vamos revisar o teste para depois aplicar no modelo discutido anteriormente.

\subsubsection{Teste $\chi^{2}$}

A ciência moderna utiliza da inferência estatística para obter e analisar informações acerca da grande quantidade de dados experimentais e observacionais disponíveis. Existem várias maneiras de se analisar um conjunto de dados, em cosmologia é comum utilizarmos o chamado teste de $\chi^{2}$.

Primeiramente vamos assumir que temos um conjunto de $N$ dados observacionais e queremos determinar $M$ parâmetros de um dado modelo de interesse. Assumimos também que 


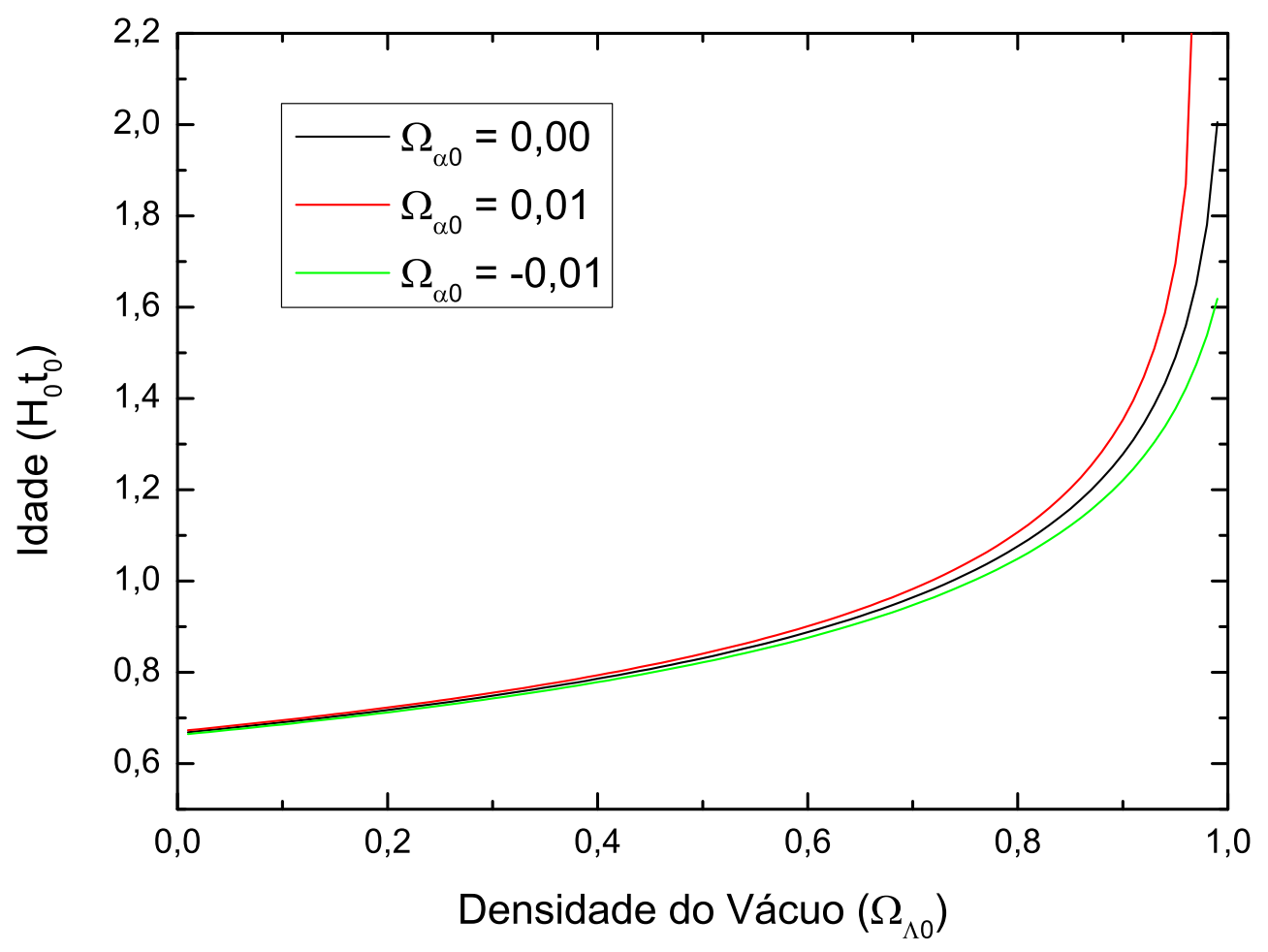

Figura 6.5: Idade do universo $H_{0} t_{0}$ do modelo plano com decaimento do vácuo. No gráfico acima mostramos o parâmetro $H_{0} t_{0}$ em função de $\Omega_{\Lambda 0}$. Note que em relação a $\Lambda C D M$ a idade cresce para $\Omega_{\alpha 0}>0$ e diminui caso contrário.

este modelo prevê uma relação funcional entre a quantidade observacionalmente medida e estes parâmetros:

$$
y(x)=y\left(x ; a_{1} \ldots a_{M}\right)
$$

onde $a_{1} \ldots a_{M}$ são os parâmetros do modelo e $x$ é uma variável independente. Vamos supor que os dados são independentes e idênticamente distribuidos (i.i.d.) em torno do modelo real " $\mathrm{y}(\mathrm{x})$ ". A probabilidade conjunta dos dados é obtido pelo produto das probabilidades individuais, que seguem uma gaussiana

$$
P \propto \prod_{i=1}^{N} \exp \left[-\frac{1}{2}\left(\frac{y_{i}-y\left(x_{i}\right)}{\sigma_{i}}\right)^{2}\right],
$$

onde $\sigma_{i}$ é o erro associado ao dado $y_{i}$. Agora queremos obter o conjunto de parâmetros deste modelo que maximiza a probabilidade acima, para isso, podemos minimizar o módulo de seu logaritmo 


$$
\sum_{i=1}^{N}\left(\frac{y_{i}-y\left(z_{i}\right)}{2 \sigma_{i}}\right)^{2}
$$

O teste de $\chi^{2}$ consiste em minimizar a seguinte expressão

$$
\chi^{2} \equiv \sum_{i=1}^{N}\left(\frac{y_{i}-y\left(z_{i}\right)}{\sigma_{i}}\right)^{2},
$$

onde definimos o $\chi^{2}$ com base na expressão anterior. A probabilidade dos dados na equação (6.64) é chamada verossimilhança, sendo o teste de $\chi^{2}$ também conhecido como método da máxima verossimilhança. Uma maneira de verificar se o ajuste é razoável é comparar o mínimo $\chi_{\min }^{2}$ obtido com o número de graus de liberdade do sistema, definido por $\nu \equiv$ $N-M$. Se estas quantidades forem da mesma ordem $\left(\chi_{\min }^{2} \approx \nu\right)$, o ajuste pode ser considerado confiável (Press et al. (2007)) ).

Para estimar a incerteza dos parâmetros podemos definir curvas de níveis $\left(\Delta \chi^{2} \equiv\right.$ $\left.\chi^{2}-\chi_{\min }^{2}\right)$ que delimitam regiões no espaço de parâmetros onde há um certo percentual $p$ da distribuição de probabilidade total. Normalmente escolhe-se 68, 27\%, 95, 45\% e 99, 73\% de confiança estatística. A tabela 6.1 mostra o valor de $\Delta \chi^{2}$ para o número de parâmetros variando de 1 a 3 . Nosso ajuste utilizou dois parâmetros livres, $\Omega_{m 0}$ e $\Omega_{\Lambda 0}$.

Tabela 6.1 - Valores de $\Delta \chi^{2}$ correspondentes à $68,27 \%, 95,45 \%$ e 99, 73\%, para diferentes números de parâmetros, tabela adaptada de Press et al. (2007), pag. 815.

\begin{tabular}{lccc}
\hline \hline Porcentagem(\%) & $m=1$ & $m=2$ & $m=3$ \\
\hline 68,27 & 1,00 & 2,30 & 3,53 \\
95,45 & 4,00 & 6,18 & 8,02 \\
99,73 & 9,00 & 11,8 & 14,2 \\
& & & \\
\hline
\end{tabular}

Neste trabalho minimizamos a seguinte função $\chi^{2}$, construída com a partir das observações discutidas no capítulo 3

$$
\chi^{2}=\sum_{1}^{580}\left[\frac{\mu_{i}-\mu\left(z_{i} ; \Omega_{m 0}, \Omega_{\Lambda 0}\right)}{\sigma_{i}}\right]+\frac{R_{e x p}-R\left(\Omega_{m 0}, \Omega_{\Lambda 0}\right)}{\sigma_{R}}+\frac{A_{e x p}-A\left(0,35 ; \Omega_{m 0}, \Omega_{\Lambda 0}\right)}{\sigma_{A}}
$$


onde $\mu_{i}$ são os dados do Union 2.1, $\mu$ é o módulo de distância, dado pela equação (3.26), $R$ é o shift parameter, dado pela equação (3.37) e $A$ é dado pela equação (3.46).

\subsubsection{Contornos de Confiança e Melhor Ajuste}

Os dados foram ajustados com base no modelo plano, utilizando a equação de Hubble (6.48) com a limitação (6.59). Primeiramente vamos verificar a influência de nosso modelo no contorno de confiança das supernovas. Observando a figura abaixo percebemos que a elipse gira na direção $\Omega_{m 0}+\Omega_{\Lambda 0}=1$, ou seja, nosso modelo se aproxima do contorno do CMB.

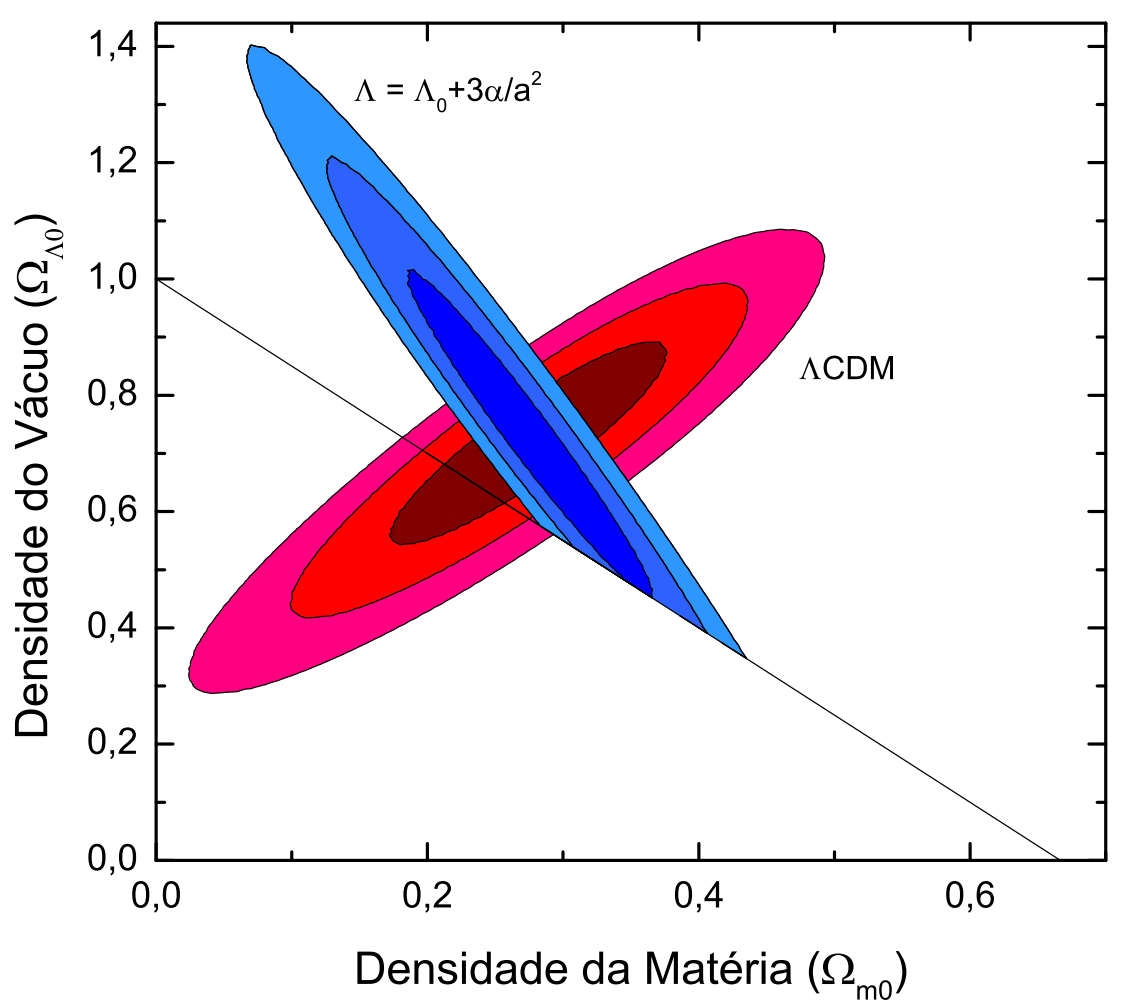

Figura 6.6: Comparação dos contornos de Confiança de SNe para o nosso modelo e $\Lambda C D M$.

A figura 6.7 mostra os contornos de confiança do nosso modelo para uma análise conjunta envolvendo os dados de SNe, CMB e BAO. O melhor ajuste obtido foi (com erros em $1 \sigma) \Omega_{m 0}=0,27 \pm 0,02, \Omega_{\Lambda 0}=0,74 \pm 0,02$ e $\Omega_{\alpha 0}=1-\Omega_{m 0}-\Omega_{\Lambda 0}=-0,01 \pm 0,03$. O valor de $\chi_{\min }^{2}$ da análise foi 562 , com $\chi_{\text {red }}^{2}=0,97$.

Deve-se notar que todo o espaço de parâmetros coberto no teste é plano, pois quere- 


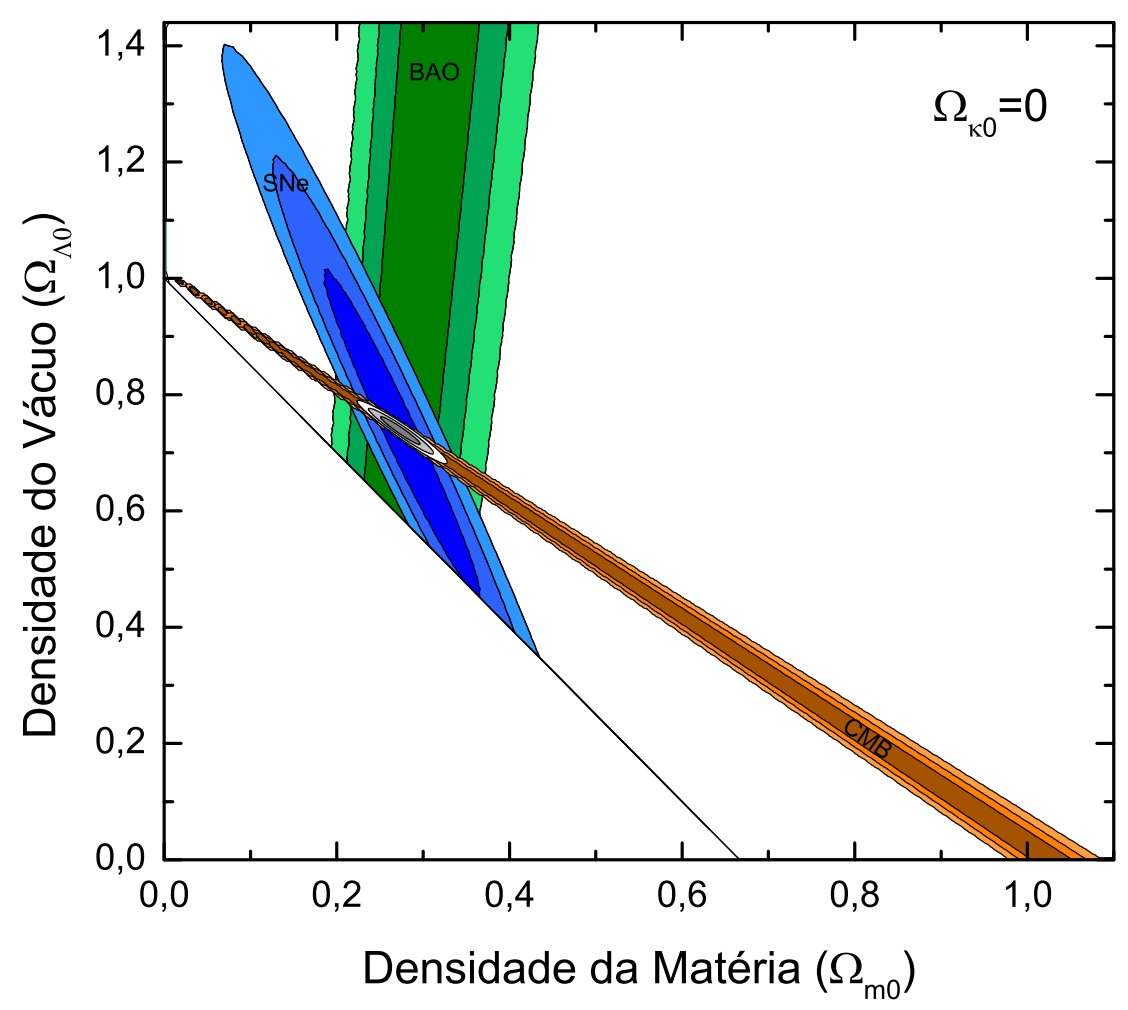

Figura 6.7: Contornos de confiança do modelo plano para os dados de SNe, CMB e BAO. Note que a contorno das supernovas se inclina na direção do contorno de CMB (compare com a figura do capítulo 3). É essa rotação que diminui a tensão SNe-CMB.

mos analisar como se modificam os contornos de confiança em relação à curvatura efetiva introduzida por $\Omega_{\alpha 0}$. A ideia básica é mostrar que a tensão SNe-CMB pode ser aliviada ou completamente resolvida pelo termo de decaimento adotado neste trabalho.

Comparando a figura 6.7 com a figura 3.7 (Suzuki et al., 2012) percebe-se que o contorno das SNe se inclinou na direção $\Omega_{m 0}+\Omega_{\Lambda 0}=1$, permitindo valores de $\Omega_{\alpha 0}$ tanto negativos quanto positivos, o contorno de CMB indica que $\Omega_{\alpha 0}$ deve ser pequeno, próximo de zero. Por outro lado, o contorno associado ao BAO agora apresenta uma razoável degenerescência com $\Omega_{\Lambda 0}$. 


\section{Conclusões e Perspectivas}

É bem conhecido que a maneira mais direta de se investigar as potencialidades de um modelo cosmológico é confrontando suas previsões com os chamados testes cosmológicos observacionais. Um dos principais testes está relacionado com a determinação teórica das distâncias de luminosidade que envolvem uma vela padrão (Supernovas do tipo Ia) e sua comparação com as observações. Tal teste fornece uma importante restrição sobre as possíveis contribuições dos principais contituintes cósmicos. Um teste complementar é o da radiação cósmica de fundo $(\mathrm{CMB})$ através das anisotropias de temperatura e o espectro angular de potência associado (WMAP, Planck, etc).

Por outro lado, sabemos que as Supernovas separadamente preferem um Universo fechado $\left(\Omega_{\kappa}>1\right)$ enquanto as observações da CMB independemente favorecem um modelo plano $\left(\Omega_{\kappa}=0\right)$, o qual está mais de acordo com o chamado paradigma inflacionário. Com o intuito de resolver essa tensão, discutimos nesta dissertação as previsões de um modelo $\operatorname{com} \Lambda(t)$, onde a variação da densidade de energia do vácuo $\left(\rho_{v}=\Lambda / 8 \pi G\right)$ foi fenomenologicamente modelada pela expressão: $\Lambda=\Lambda_{0}+3 \alpha / a^{2}$, onde $\Lambda_{0}$ é o termo de vácuo usual, $\alpha$ é um parâmetro livre e $a(t)$ o fator de escala.

A principal vantagem do modelo é poder ser plano para vários valores dos parâmetros $\Omega_{m 0}$ e $\Omega_{\Lambda 0}$, sem a limitação padrão, ou seja, $\Omega_{m 0}+\Omega_{\Lambda 0}=1$, tal como ocorre no chamado modelo de concordância cósmica $(\Lambda C D M)$. A razão básica para este comportamento é que o parâmetro $\Omega_{\alpha 0}$, definido em (6.41), se comporta como uma curvatura efetiva.

O modelo apresentado simula a dinâmica de $\Lambda C D M$ pois o parâmetro de Hubble é dado por (ver equação (6.48) ):

$$
\frac{H^{2}}{H_{0}^{2}}=\Omega_{m e f 0}\left(\frac{a_{0}}{a}\right)^{3}+\Omega_{\Lambda 0}+\Omega_{\kappa e f 0}\left(\frac{a_{0}}{a}\right)^{2},
$$


onde:

$$
\Omega_{m e f 0} \equiv \Omega_{m 0}-2 \Omega_{\alpha 0}
$$

$\mathrm{e}$

$$
\Omega_{\kappa e f 0} \equiv 1-\Omega_{m e f 0}-\Omega_{\Lambda 0}=\Omega_{\kappa 0}+3 \Omega_{\alpha 0} .
$$

Os testes observacionais utilizados para obter limites nos parâmetros cosmológicos foram $\mathrm{SNe}, \mathrm{CMB}$ e $\mathrm{BAO}$, discutidos no capítulo 3. Os resultados obtidos, bem como a comparação com os dados de Supernovas e do satélite Planck estão apresentados na tabela 7.1 .

Tabela 7.1 - Comparação entre os resultados do Planck (Ade et al., 2013), Supernova Cosmology Project (Suzuki et al., 2012) e este trabalho.

\begin{tabular}{lccc}
\hline \hline \multicolumn{1}{c}{ Resultados } & Supernova Cosmology Project & Satélite Planck & Este trabalho \\
\hline$\Omega_{m 0}$ & $0,272 \pm 0,014$ & $0,314 \pm 0,020$ & $0,27 \pm 0,02$ \\
$\Omega_{\Lambda 0}$ & $0,726 \pm 0,014$ & $0,686 \pm 0,020$ & $0,74 \pm 0,02$ \\
$\Omega_{\alpha 0}$ & 0 & 0 & $-0,01 \pm 0,03$ \\
$\Omega_{v 0}=\Omega_{\Lambda 0}+\Omega_{\alpha 0}$ & $0,726 \pm 0,014$ & $0,686 \pm 0,020$ & $0,73 \pm 0,04$ \\
\hline
\end{tabular}

Naturalmente, o modelo é compatível com $\Omega_{\alpha 0}=0$, ou seja, ele não se afasta muito de $\Lambda C D M$ padrão. No entanto, para $\Omega_{\alpha 0} \neq 0$, a tensão entre os dados de SNe Ia e CMB foi aliviada pois o modelo é plano para os 2 conjuntos de dados e de medida não nula, pois o espaço disponível no plano $\Omega_{m 0}-\Omega_{\Lambda 0}$ (supondo $\left.\Omega_{\kappa} \equiv 0\right)$ ainda acomoda uma considerável variação dos parâmetros cosmológicos (ver Figura 6.7).

Alguns aspectos desse trabalho deverão ser investigados no futuro próximo, dentre os quais destacamos:

- Uma investigação da tensão Supernova-CMB supondo uma variação mais geral de $\Lambda(t)$ do que a considerada neste trabalho. Por exemplo, para manter o nível de simplicidade da descrição fenomenológica, poderemos considerar uma dependência do tipo: $\Lambda=\Lambda_{0}+3 \alpha / a^{n}$, onde $n$ é um número positivo. A potência $n$ deve afetar consideravelmente vários aspectos do modelo, incluindo o redshift de transição e o melhor ajuste dos parâmetros livres $\left(\Omega_{m e f 0}\right.$ e $\left.\Omega_{\Lambda 0}\right)$. 
- Introdução dos bárions. No presente trabalho consideramos uma interação no setor escuro. Isto significa que os bárions são identicamente conservados e portanto dariam um contribuição do tipo $\rho_{b}=\rho_{b o}(1+z)^{3}$. Como $\Lambda(t)$ interage apenas no setor escuro, é interessante discutir se a tensão Supernova-CMB seria mais alivida (ou não) com a introdução dos bários, para os quais $\Omega_{b} \sim 0,045$ (Ade et al., 2013).

- Embora resolvendo o problema da idade total do Universo (a idade total prevista não é muito diferente do caso $\Lambda C D M$ - ver figura 6.5). No entanto, não é claro se o modelo poderia ser compatível com um problema mais restritivo, tal como, o problema da idade em altos redshifts (Alcaniz e Lima, 1999; Alcaniz et al.,, 2003; Cunha e Santos, 2004). Para esse problema seria interessante também investigar o caso mais geral, isto é, $\Lambda \propto a^{-n}$.

Finalmente, no contexto do presente modelo, nos parece importante também discutir o problema geral de evolução das perturbações para comparar com os dados existentes e futuros. Em particular, os vínculos provenientes da função de crescimento (nos regimes linear e não linear) deveriam ser investigados para verificar se o modelo é fisicamente viável. A princípio, tais resultados deveriam ser analisados conjuntamente com outros testes cosmológicos complementares envolvendo, por exemplo: lentes gravitacionais, dados de raios-X de aglomerados, fontes de radio compactas, efeito Sunyaev-Zel'dovich e objetos velhos em altos redshifts. Uma análise conjunta envolvendo testes independentes e a consequente limitação no espaço de parâmetros, ajudariam a decidir se este cenário com $\Lambda(t)$ poderá se tornar uma alternativa realística para o atual modelo de concordância cósmica $(\Lambda C D M)$. 


\section{Referências Bibliográficas}

Abdel-Rahman A.-M. M., Singularity-Free Decaying-Vacuum Cosmologies, Physical Review D, 1992, vol. 45, p. 3497

Abdussattar Vishwakarma R. G., Some FRW models with variable $G$ and $\Lambda$, Classical and Quantum Gravity, 1997, vol. 14, p. 945

Ade P. A. R., et al., Planck 2013 Results. XVI. Cosmological Parameters, arXiv:1303.5076, 2013

Alcaniz J. S., Lima J. A. S., New Limits on $\Omega_{\Lambda}$ and $\Omega_{M}$ from Old Galaxies at High Redshift, The Astrophysical Journal Letters, 1999, vol. 521, p. L87

Alcaniz J. S., Lima J. A. S., Interpreting Cosmological Vacuum Decay, Physical Review D, 2005, vol. 72 , p. 063516

Alcaniz J. S., Lima J. A. S., Cunha J. V., Cosmological implications of APM 08279+5255, an old quasar at $\mathrm{z}=3.91$, Monthly Notices of the Royal Astronomical Society, 2003, vol. 340 , p. L39

Alpher R. A., Herman R. C., Evolution of the Universe, Nature, 1948, vol. 162, p. 774

Amanullah R., et al., Spectra and Hubble Space Telescope Light Curves of Six Type Ia Supernovae at $0.511<z<1.12$ and the Union2 Compilation, The Astrophysical Journal, 2010, vol. 716, p. 712

Amendola L., Kainulainen K., Marra V., Quartin M., Large-Scale Inhomogeneities May Improve The Cosmic Concordance Of Supernovae, Physical Review Letters, 2010, vol. 105, p. 121302 
Anderson L., et al., The clustering of galaxies in the SDSS-III Baryon Oscillation Spectroscopic Survey: Baryon Acoustic Oscillations in the Data Release 9 Spectroscopic Galaxy Sample, Monthly Notices of the Royal Astronomical Society, 2013, vol. 428, p. 1036

Arbab A. I., Cosmological Models with Variable Cosmological and Gravitational Constants and Bulk Viscous Models, General Relativity and Gravitation, 1997, vol. 29, p. 61

Arbab A. I., Abdel-Rahman A. M. M., Nonsingular cosmology with a time-dependent cosmological term, Physical Review D, 1994, vol. 50, p. 7725

Beesham A., Cosmological models with a variable cosmological term and bulk viscous models, Physical Review D, 1993, vol. 48, p. 3539

Beesham A., Bianchi type I cosmological models with variable G and A, General Relativity and Gravitation, 1994, vol. 26, p. 159

Bennett C. L., et al., Nine-Year Wilkinson Microwave Anisotropy Probe (WMAP) Observations: Final Maps and Results, arXiv:1212.5225v2, 2013

Bento M. C., Bertolami O., Sen A. A., Generalized Chaplygin Gas, Accelerated Expansion and Dark-Energy-Matter Unification, Physical Review D, 2002, vol. 66, p. 043507

Berman M. S., Cosmological models with a variable cosmological term, Physical Review D, 1991, vol. 43, p. 1075

Berman M. S., Som M. M., Brans-Dicke models with time-dependent cosmological term, International Journal of Theoretical Physics, 1990, vol. 29, p. 1411

Bertolami O., Time-dependent cosmological term, Nuovo Cimento B, 1986, vol. 93, p. 36

Beutler F., et al., The 6dF Galaxy Survey: baryon acoustic oscillations and the local Hubble constant, Monthly Notices of the Royal Astronomical Society, 2011, vol. 416, p. 3017

Birkel M., Sarkar S., Nucleosynthesis bounds on a time-varying cosmological constant, Astroparticle Physics, 1997, vol. 6, p. 197 
Blake C., et al., The WiggleZ Dark Energy Survey: joint measurements of the expansion and growth history at $\mathrm{z}<1$, Monthly Notices of the Royal Astronomical Society, 2012, vol. 425 , p. 405

Bondi H., Cosmology. 2nd ed. Cambridge University Press, 1968, 182 p.

Caldera-Cabral G., Maartens R., Ureña-López L. A., Dynamics of Interacting Dark Energy, Physical Review D, 2009, vol. 79, p. 063518

Calvao M. O., Lima J. A. S., Waga I., On the thermodynamics of matter creation in cosmology, Physics Letters A, 1992, vol. 162, p. 223

Calvao M. O., Oliveira H. P., Pavón D., Salim J. M., Dissipative cosmology with decaying vacuum energy, Physical Review D, 1992, vol. 45, p. 3869

Canuto V., Hsieh S. H., Adams P. J., Scale-Covariant Theory of Gravitation and Astrophysical Applications, Physical Review Letters, 1977, vol. 39, p. 429

Carvalho F. C., Alcaniz J. S., Lima J. A. S., Silva R., Scalar-Field Dominated Cosmology with a Transient Acceleration Phase, Physical Review Letters, 2006, vol. 97, p. 081301

Carvalho J. C., Lima J. A. S., Waga I., Cosmological Consequences of a Time-Dependent $\Lambda$ Term, Physical Review D, 1992, vol. 46, p. 2404

Chaplygin S. A., On Gas Jets, Sci. Mem. Moscow Univ. Math. Phys., 1904, vol. 21, p. 1

Chen W., Wu Y., Implications Of A Cosmological Constant Varying As $R^{-2}$, Physics Letters D, 1990, vol. 41, p. 695

Chevallier M., Polarski D., Accelerating Universes With Scaling Dark Matter, International Journal of Modern Physics D, 2001, vol. 10, p. 213

Conley A., et al., Supernova Constraints and Systematic Uncertainties from the First 3 Years of the Supernova Legacy Survey, The Astrophysical Journal Supplement Series, 2011, vol. 192, p. 1

Cunha J. V., Testes Cinemáticos em Cosmologias com Decaimento do Vácuo, Natal: Universidade Federal do Rio Grande do Norte, 2002, Dissertação de Mestrado, 89 p. 
Cunha J. V., Kinematic constraints to the transition redshift from supernovae type Ia union data, Physical Review D, 2009, vol. 79, p. 047301

Cunha J. V., Lima J. A. S., Transition redshift: new kinematic constraints from supernovae, Monthly Notices of the Royal Astronomical Society, 2008, vol. 390, p. 210

Cunha J. V., Santos R. C., The Existence of an old quasar at z $=3.91$ and its implications for $\Lambda(t)$ deflationary cosmologies, International Journal of Modern Physics D, 2004, vol. 13 , p. 1321

Davis T. M., et al., Scrutinizing exotic cosmological models using ESSENCE supernova data combined with other cosmological probes, The Astrophysical Journal, 2007, vol. 666 , p. 716

de Sitter W., On Einstein's Theory of Gravitation and its Astronomical Consequences, Monthly Notices of the Royal Astronomical Society, 1917, vol. 78, p. 3

Demiański M., History of the Cosmological Constant, Annals of Physics, 2000, vol. 9, p. 278

Dev A., Alcaniz J. S., Jain D., Cosmological consequences of a Chaplygin gas dark energy, Physical Review D, 2003, vol. 67, p. 023515

Dodelson S., Modern Cosmology. 1st ed. Academic Press, 2003, 440 p.

Dvali G., Gabadadze G., Porrati M., 4D gravity on a brane in 5D Minkowski space, Physics Letters B, 2000, vol. 485, p. 208

Einstein A., Die Feldgleichung der Gravitation (The Field Equations of Gravitation), Sitzungsberichte, Königlich Preussische Akademie der Wissenschaften, 1915, vol. 2, p. 844

Einstein A., Die Grundlage der Allgemeinen Relativitätstheorie (The Foundation of the General Theory of Relativity), Annalen der Physik, 1916, vol. 49, p. 769

Einstein A., Kosmologische Betrachtungen zur Allgemeinen Relativitätstheorie (Cosmological Considerations in the General Theory of Relativity), Sitzungsberichte, Königlich Preussische Akademie der Wissenschaften, 1917, vol. 1, p. 142 
Einstein A., Zum Kosmologischen Problem der Allgemeinen Relativitätstheorie (Cosmological Problem of General Relativity), Sitzungsberichte der Preussischen Akademie der Wissenschaften, 1931, pp 235-237

Einstein A., de Sitter W., On the Relation Between the Expansion and the Mean Density of the Universe, Procedings of the National Academy of Science, 1932, vol. 18, p. 213

Eisenstein D. J., et al., Detection of the Baryon Acoustic Peak in the Large-Scale Correlation Function of SDSS Luminous Red Galaxies, The Astrophysical Journal, 2005, vol. 633 , p. 560

Eisenstein D. J., Seo H.-J., White M., On the Robustness of the Acoustic Scale in the Low-Redshift Clustering of Matter, The Astrophysical Journal, 2007, vol. 664, p. 660

Ellis G. F. R., On the Definition of Distance in General Relativity: I. M. H. Etherington (Philosophical Magazine ser. 7, vol. 15, 761 (1933)), General Relativity and Gravitation, 2007, vol. 39, p. 1047

Endo M., Fukui T., The Cosmological Term and a Modified Brans-Dicke Cosmology , General Relativity and Gravitation, 1977, vol. 8, p. 833

Etherington I. M. H., On the Definition of Distance in General Relativity, Philosophical Magazine ser. 7, 1933, vol. 15, p. 761

Freese K., Adams F. C., Frieman J. A., Mottola E., Cosmology with decaying vacuum energy, Nuclear Physics B, 1987, vol. 287, p. 797

Friedmann A., Über die Krümmung des Raumes (On the curvature of space), Zeitschrift für Physik, 1922, vol. 10, p. 377

Friedmann A., Über die Möglichkeit einer Welt mit konstanter negativer Krümmung des Raumes (On the Possibility of a World with Constant Negative Curvature of Space), Zeitschrift für Physik, 1924, vol. 21, p. 326

Gasperini M., Decreasing vacuum temperature: A thermal approach to the cosmological constant problem, Physics Letters B, 1987, vol. 194, p. 347

Grøn O., Repulsive Gravitation and Inflationary Universe Models, American Journal of Physics, 1986, vol. 54, p. 46 
Groot S. R., Leeuwen W. A., van Weert C. G., Relativistic kinetic theory: principles and applications. 1st ed. North-Holland, 1980, 417 p.

Guimarães A. C. C., Cunha J. V., Lima J. A. S., Bayesian Analysis and Constraints on Kinematic Models from Union SNIa, Journal of Cosmology and Astroparticle Physics, 2009, vol. 2009, p. 010

Guimarães A. C. C., Lima J. A. S., Could the cosmic acceleration be transiest? A cosmographic evaluation, Classical and Quantum Gravity, 2011, vol. 28, p. 125026

Gunzig E., Maartens R., Nesteruk A. V., Inflationary cosmology and thermodynamics, Classical and Quantum Gravity, 1998, vol. 15, p. 923

Hinshaw G., et al., Nine-Year Wilkinson Microwave Anisotropy Probe (WMAP) Observations: Cosmological Parameter Results, arXiv:1212.5226v2, 2013

Hiscock W. A., Quantum instabilities and the cosmological constant, Physics Letters B, 1986, vol. 166, p. 285

Holanda J. V., Lima J. A. S., Ribeiro M. B., Testing the Distance-Duality Relation with Galaxy Clusters and Type Ia Supernovae, The Astrophysical Journal Letters, 2010, vol. 722 , p. L233

Holanda J. V., Lima J. A. S., Ribeiro M. B., Cosmic distance duality relation and the shape of galaxy clusters, Astronomy \& Astrophysics, 2011, vol. 528, p. L14

Hoyle F., Burbidge G., Narlikar J. V., On the Hubble constant and the cosmological constant, Monthly Notices of the Royal Astronomical Society, 1997, vol. 286, p. 173

Hu W., Sugiyama N., Small-Scale Cosmological Perturbations: An Analytic Approach, The Astrophysical Journal, 1996, vol. 471, p. 542

Hubble E. P., A Relation Between Distance and Radial Velocity Among Extra-Galactic Nebulae, Proceedings of the National Academy of Sciences, 1929, vol. 15, p. 168

Huterer D., Turner M. S., Probing dark energy: Methods and strategies, Physical Review D, 2001, vol. 64, p. 123527 
Jesus J. F., Santos R. C., Alcaniz J. S., Lima J. A. S., New Coupled Quintessence Cosmology, Physical Review D, 2008, vol. 78, p. 063514

John M. V., Joseph K. B., A low matter density decaying vacuum cosmology from a complex metric, Classical and Quantum Gravity, 1997, vol. 14, p. 1115

John M. V., Joseph K. B., Generalized Chen-Wu Type Cosmological Model, Physical Review D, 2000, vol. 61, p. 087304

Kalligas D., Wesson P., Everitt C. W. F., Flat FRW models with variable G and $\Lambda$, General Relativity and Gravitation, 1992, vol. 24, p. 351

Kalligas D., Wesson P. S., Everitt C. W. F., Bianchi type I cosmological models with variable $\mathrm{G}$ and $\Lambda$ : A comment, General Relativity and Gravitation, 1995, vol. 27, p. 645

Kamenshchik A., Moschella U., Pasquier V., An Alternative to Quintessence, Physics Letters B, 2001, vol. 511, p. 265

Kazanas D., Dynamics of the Universe and Spontaneous Symmetry Breaking, Astrophysical Journal Letters, 1980, vol. 241, p. L59

Kolb E. W., Turner M. S., The early universe.. 1st ed. Westview Press, 1990, 592 p.

Landau L. D., Lifshitz E. M., Fluid Mechanics. 2nd ed. Elsevier, 1984, 539 p.

Lau Y. K., The Large Number Hypothesis and Einstein's Theory of Gravitation , Australian Journal of Physics, 1985, vol. 38, p. 547

Lazkoz R., Majerotto E., Cosmological constraints combining H(z), CMB shift and SNIa observational data, Journal of Cosmology and Astroparticle Physics, 2007, vol. 2007, p. 015

Lima J. A. S., Thermodynamics of decaying vacuum cosmologies, Physical Review D, 1996, vol. 54 , p. 2571

Lima J. A. S., Alternative dark energy models: an overview, Brazilian Journal of Physics, 2004, vol. 34, p. 194

Lima J. A. S., Alcaniz J. S., Cunha J. V., A simplified approach for Chaplygin-type cosmologies, Astroparticle Physics, 2008, vol. 30, p. 196 
Lima J. A. S., Basilakos S., From de Sitter to de Sitter: A New Cosmic Scenario without Dark Energy, arXiv:1106.1938, 2011

Lima J. A. S., Busti V. C., Santos R. C., Extended Dyer-Roeder Approach Improves the Cosmic Concordance Model, arXiv:1301.5360, 2013

Lima J. A. S., Calvão M. O., Waga I., Cosmology, Thermodynamics and Matter Creation. World Scientific, Singapore, 1990

Lima J. A. S., Carvalho J. C., Dirac's cosmology with varying cosmological constant, General Relativity and Gravitation, 1994, vol. 26, p. 909

Lima J. A. S., Cunha J. V., Alcaniz J. S., Simplified quartessence cosmology, Astroparticle Physics, 2009, vol. 31, p. 233

Lima J. A. S., Jesus J. F., Oliveira F. A., CDM Accelerating Cosmology as an Alternative to $\Lambda$ CDM model, Journal of Cosmology and Astroparticle Physics, 2010, vol. 11, p. 027

Lima J. A. S., Jesus J. F., Santos R. C., Gill M. S. S., Is the transition redshift a new cosmological number?, arXiv:1205.4688v2, 2012

Lima J. A. S., Maia J. M. F., Deflationary cosmology with decaying vacuum energy density, Physical Review D, 1994, vol. 49, p. 5597

Lima J. A. S., Silva A. I., Viegas S. M., Is the radiation temperature redshift relation of the standard cosmology in accordance with the data?, Monthly Notices of the Royal Astronomical Society, 2000, vol. 312, p. 747

Lima J. A. S., Trodden M., Decaying vacuum energy and deflationary cosmology in open and closed universes, Physical Review D, 1996, vol. 53, p. 4280

Lima J. A. S., Zanchin V., Brandenberger R., On The Newtonian Cosmology equations With Pressure, Monthly Notices of the Royal Astronomical Society, 1997, vol. 291, p. L1

Linder E. V., Probing Gravitation, Dark Energy, and Acceleration, Physical Review D, 2004, vol. 70, p. 023511 
Lopez J. L., Nanopoulos D. V., A New Cosmological Constant Model, Modern Physics Letters A, 1996, vol. 11, p. 1

McCrea W. H., Milne E. A., Newtonian universes and the curvature of space, The Quarterly Journal of Mathematics, 1934, pp 73-80

Maia M. D., Silva G. S., Geometrical constraints on the cosmological constant, Phys. Rev. D, 1994, vol. 50, p. 7233

Martin J., Everything You Always Wanted to Know About the Cosmological Constant Problem (But Were Afraid to Ask), arXiv:1205.3365v1, 2012

Matyjasek J., Cosmological models with a time-dependent $\Lambda$ term, Physical Review D, 1995, vol. 51, p. 4154

Méndez V., Pavón D., Expanding models with a varying cosmological term and bulk stress, General Relativity and Gravitation, 1996, vol. 28, p. 679

Moffat J. W., Lagrangian formulation of a solution to the cosmological constant problem , arXiv:astro-ph/9608202, 1996

Nishimichi T., et al., Characteristic Scales of Baryon Acoustic Oscillations from Perturbation Theory: Non-linearity and Redshift-Space Distortion Effects, Publications of the Astronomical Society of Japan, 2007, vol. 59, p. 1049

Nobbenhuis S., The Cosmological Constant Problem, an Inspiration for New Physics, arXiv:gr-qc/0609011, 2006

Olson T. S., Jordan T. F., Ages of the Universe for decreasing cosmological constants, Physical Review D, 1987, vol. 35, p. 3258

Overduin J. M., Cooperstock F. I., Evolution of the Scale Factor With a Variable Cosmological Term, Physical Review D, 1998, vol. 58, p. 043506

Overduin J. M., Wesson P. S., Bowyer S., Constraints on decaying neutrinos from the far ultraviolet extragalactic background light, Astrophysical Journal, 1993, vol. 404, p. 460

Ozer M., Taha M., A Possible Solution To The Main Cosmological Problems, Physics Letters B, 1986, vol. 171, p. 363 
Ozer M., Taha M., A Model Of The Universe Free Of Cosmological Problems, Nuclear Physics, 1987, pp 776-796

Padmanabhan N., Xu X., Eisenstein D. J., Scalzo R., Cuesta A. J., Mehta K. T., Kazin E., A 2 per cent distance to $\mathrm{z}=0.35$ by reconstructing baryon acoustic oscillations $\mathrm{I}$. Methods and application to the Sloan Digital Sky Survey, Monthly Notices of the Royal Astronomical Society, 2012, vol. 427, p. 2132

Pavón D., Nonequilibrium Fluctuations in Cosmic Vacuum Decay, Physical Review D, 1991, vol. 43, p. 375

Peebles P. J. E., Principles of Physical Cosmology. 1st ed. Princeton University Press, 1993, 718 p.

Penzias A. A., Wilson R. W., A Measurement of Excess Antenna Temperature at 4080Mc/s, The Astrophysical Journal, 1965, vol. 142, p. 419

Perlmutter S., et al., Measurements of $\Omega$ and $\Lambda$ From 42 High-Redshift Supernovae, The Astrophysical Journal, 1999, vol. 517, p. 565

Phillips M. M., The absolute magnitudes of Type IA supernovae, The Astrophysical Journal, 1993, vol. 413, p. L105

Pigozzo C. B. M., Testes de Concordância em Modelos Cosmológicos com Interação no Setor Escuro, Salvador: Universidade Federal da Bahia, 2010, Tese de Doutorado, 96 p.

Press W. H., Teukolsky S. A., Vetterling W. T., Flannery B. P., Numerical Recipes: The Art of Scientific Computing. 3rd ed. Cambridge University Press, 2007, 1235 p.

Prigogine I., Geheniau J., Gunzig E., Nardone P., Thermodynamics and Cosmology, General Relativity and Gravitation, 1989, vol. 21, p. 767

Rajeev S. G., Why is the cosmological constant small?, Physics Letters B, 1983, vol. 125, p. 144

Reuter M., Wetterich C., Time evolution of the cosmological constant, Physics Letters B, 1987, vol. 188 , p. 38 
Riess S., et al., Observational Evidence From Supernovae for an Accelerating Universe and a Cosmological Constant, The Astronomical Journal, 1998, vol. 116, p. 1009

Robertson H. P., Kinematics and World Structure, Astrophysical Journal, 1935, vol. 82, p. 284

Rubin V. C., Burstein D., Ford W. K., Thonnard N., Rotation Velocities of 16 Sa Galaxies and a Comparison of Sa, Sb, and Sc Rotation Properties, Astrophysical Journal, 1985, vol. 289 , p. 81

Rubin V. C., Ford W. K., Rotation of the Andromeda Nebula from a Spectroscopic Survey of Emission Regions, Astrophysical Journal, 1970, vol. 159, p. 379

Sakurai J. J., Modern Quantum Mechanics. 1st ed. Addison Wesley, 1993, 500 p.

Salim J. M., Waga I., Thermodynamic constraints on a time-dependent Lambda model (cosmology), Classical and Quantum Gravity, 1993, vol. 10, p. 1767

Sandvik H. B., Tegmark M., Zaldarriaga M., The end of unified dark matter?, Physical Review D, 2004, vol. 69, p. 123524

Schrödinger E., The Proper Vibrations of the Expanding Universe, Physica, 1939, vol. 6, p. 899

Shafieloo A., Sahni V., Starobinsky A., Is cosmic acceleration slowing down?, Physical Review D, 2009, vol. 80, p. 101301

Silveira V., Waga I., Decaying $\Lambda$ cosmologies and power spectrum, Physical Review D, 1994, vol. 50, p. 4890

Silveira V., Waga I., Cosmological properties of a class of $\Lambda$ decaying cosmologies, Physical Review D, 1997, vol. 56, p. 4625

Sistero R. F., Cosmology with G and $\Lambda$ coupling scalars, General Relativity and Gravitation, 1991, vol. 23, p. 1265

Smoot G. F., Scott D., The Cosmic Background Radiation, arXiv:9603157, 1996

Sotiriou T. P., Faraoni V., f(R) theories of gravity, Reviews of Modern Physics, 2010, vol. 82 , p. 451 
Spindel P., Brout R., Entropy production from vacuum decay, Physics Letters B, 1994, vol. 320 , p. 241

Straumann N., The History of the Cosmological Constant Problem, arXiv:gr-qc/0208027, 2002

Suzuki N., et al., The Hubble Space Telescope Cluster Supernova Survey: V. Improving the Dark Energy Constraints Above $z>1$ and Building an Early-Type-Hosted Supernova Sample, The Astrophysical Journal, 2012, vol. 746, p. 1

Torres L. F. B., Waga I., Decaying lambda cosmologies and statistical properties of gravitational lenses, Monthly Notices of the Royal Astronomical Society, 1996, vol. 279, p. 712

Turner M. S., Riess A. G., Do Type Ia Supernovae Provide Direct Evidence For Past Deceleration of the Universe?, The Astrophysical Journal, 2002, vol. 569, p. 18

Vargas C. Z., Hipólito-Ricaldi W. S., Zimdahl W., Perturbations for Transient Acceleration, Journal of Cosmology and Astroparticle Physics, 2012, vol. 32, p. 1

Visser M., Cosmography: Cosmology without the Einstein equations, General Relativity and Gravitation, 2004, vol. 37, p. 1541

Waga I., Decaying vacuum flat cosmological models: Expressions for some observable quantities and their properties, Astrophysical Journal, 1993, vol. 414, p. 436

Walker A. G., On Milne's Theory of World Structure, Procedings of the London Mathematical Society, Series 2, 1936, vol. 42, p. 90

Wang P., Meng X., Can Vacuum Decay in our Universe, Classical and Quantum Gravity, 2005, vol. 22, p. 283

Weinberg S., Gravitation and Cosmology: Principles ans Aplications of the General Theory of Relativity. 1st ed. John Wiley \& Sons, 1972, 657 p.

Weinberg S., The Cosmological Constant Problem, Reviews of Modern Physics, 1989, vol. 61 , p. 1

Weinberg S., Cosmology. Oxford University Press, 2008, 593 p. 
Wetterich C., The Cosmon model for an asymptotically vanishing time dependent cosmological constant, Astronomy \& Astrophysics, 1995, vol. 301, p. 321

Zimdahl W., Dark Energy: A Unifying View, International Journal of Modern Physics D, 2008, vol. 17 , p. 651

Zimdahl W., Models of Interacting Dark Energy, arXiv:astro-ph/1204.5892, 2012

Zwicky F., Die Rotverschiebung von extragalaktischen Nebeln (The redsifht of Extragalactic Nebulae), Helvetica Physica Acta, 1933, vol. 6, p. 110 